\title{
A semi-empirical airfoil stall noise model based on surface pressure measurements
}

\author{
Bertagnolio, Franck; Aagaard Madsen , Helge; Fischer, Andreas; Bak, Christian
}

Published in:

Journal of Sound and Vibration

Link to article, DOI:

10.1016/j.jsv.2016.09.033

Publication date:

2017

Document Version

Peer reviewed version

Link back to DTU Orbit

Citation (APA):

Bertagnolio, F., Aagaard Madsen, H., Fischer, A., \& Bak, C. (2017). A semi-empirical airfoil stall noise model based on surface pressure measurements. Journal of Sound and Vibration, 387, 127-162.

https://doi.org/10.1016/j.jsv.2016.09.033

\section{General rights}

Copyright and moral rights for the publications made accessible in the public portal are retained by the authors and/or other copyright owners and it is a condition of accessing publications that users recognise and abide by the legal requirements associated with these rights.

- Users may download and print one copy of any publication from the public portal for the purpose of private study or research.

- You may not further distribute the material or use it for any profit-making activity or commercial gain

- You may freely distribute the URL identifying the publication in the public portal

If you believe that this document breaches copyright please contact us providing details, and we will remove access to the work immediately and investigate your claim. 


\title{
A Semi-Empirical Airfoil Stall Noise Model Based on Surface Pressure Measurements
}

\author{
Franck Bertagnolio ${ }^{\mathrm{a}, *}$, Helge Aa. Madsen ${ }^{\mathrm{a}}$, Andreas Fischer ${ }^{\mathrm{a}}$, Christian Bak ${ }^{\mathrm{a}}$ \\ ${ }^{a}$ DTU Wind Energy \\ 5 \\ Technical University of Denmark \\ DTU Risø Campus, Frederiksborgvej 399 \\ DK-4000 Roskilde, Denmark
}

\begin{abstract}
This work is concerned with the experimental study of airfoil stall and the modelling of stall noise. Using pressure taps and high-frequency surface pressure microphones flushmounted on airfoils measured in wind tunnels and on an operating wind turbine blade, the characteristics of stall are analyzed. This study shows that the main quantities of interest, namely convection velocity, spatial correlation and surface pressure spectra, can be scaled highlighting the universal nature of stall independently of airfoil shapes and flow conditions, although within a certain range of experimental conditions. Two main regimes for the scaling of the correlation lengths and the surface pressure spectra, depending on the Reynolds number of the flow can be distinguished. These results are used to develop a model for the surface pressure spectra within the detached flow region valid for Reynolds numbers ranging from $1 \times 10^{6}$ to $6 \times 10^{6}$. Subsequently, this model is used to derive a model for stall noise. Modelled noise spectra are compared with experimental data measured in anechoic wind tunnels with reasonably satisfactory agreement.
\end{abstract}

Keywords: Airfoil Stall, Aeroacoustics, Surface Pressure, Noise Model, Turbulence,

Wind Tunnel Measurements, Wind Turbine

\section{Introduction}

Airfoil stall occurs when the streamlines of a fluid flow around an airfoil do no longer smoothly follow its contour (see Figure 1). This results from the angle of attack (AoA), which measures the angle between the main flow direction in the far-field and 
the airfoil chord, becoming larger than a critical value. The flow separates from the airfoil surface as a recirculating region, usually initiating at the trailing edge, develops on the upper side of the airfoil section. It is a result of the adverse pressure gradient and the associated deceleration of the fluid along the convex airfoil surface. With increasing AoA, separation rapidly propagates over the entire airfoil chord yielding an abrupt loss of lift. Furthermore, at high Reynolds numbers the vortical structures within the separated flow region are unstable and the flow is turbulent.
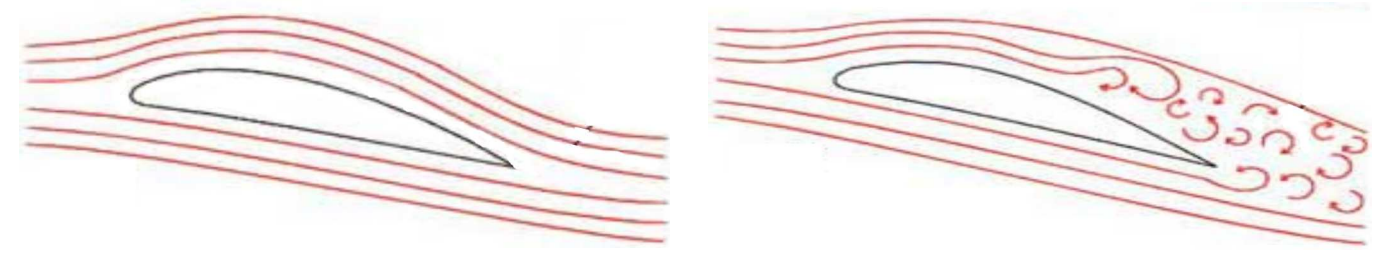

Figure 1: Attached flow (left) and stalled flow (right) around an airfoil

Although an important flow characteristic, airfoil stall has been less intensively studied than attached flows. The main reason lies probably in the fact that engineering devices using aerodynamic lift (e.g. aircraft, fans, turbines...) are commonly designed to operate outside stalled flow conditions since these usually have an unfavorable impact on their efficiency. One notable exception is a stall-regulated wind turbine for which stall is used to limit power production at high wind speeds. Early experimental studies date back to the rapid advances in aeronautics in the middle of the $20^{\text {th }}$ century $[1,2]$ and a number of empirical methods or simulation tools have been subsequently developed to predict stall occurence $[3,4]$. Dynamic stall has been more extensively studied as it is an important phenomenon in aeronautical applications that can potentially trigger aero-elastic instabilities (see the review article by McCroskey [5]). More recently, the emergence of supercomputing allowed intensive numerical Large Eddy Simulations of the stall phenomenon $[6,7]$.

As mentioned above, stall is characterized by turbulent vortices developing in the separated flow over the suction side of an airfoil and subsequently convecting into the wake. These vortices are interacting with the airfoil surface, leaving a footprint in the form of turbulent surface pressure (SP) fluctuations and are therefore producing 
noise. This type of mechanism is denoted as 'self-noise' in the aeroacoustic terminology since the vortices generated by the airfoil itself are responsible for the noise generation by interaction with the airfoil surface. It is more specifically referred to as airfoil stall noise, but also sometimes as separation noise when the flow remains attached on a significant part of the airfoil chord (as opposed to 'deep-stall' when the flow separation stretches over the entire chord). Airfoil stall noise has been investigated experimentally and acoustic measurements are usually performed in dedicated anechoic wind tunnels $[8,9,10,11,12,13,14]$. A few models have been devised, the most popular one probably being the so-called BPM model [8]. This model is based on acoustic measurements of a NACA-0012 airfoil and it is parametrized using measured boundary layer properties for this particular airfoil, such as boundary layer and displacement thicknesses. It is then assumed that the model can be extended to different airfoil shapes, assuming that identical boundary layer properties yield identical radiated noise. Moreau et al [11] conducted measurements of a NACA0012 and a NACA65-1210 airfoil in the anechoic wind tunnel facility at ECL (Lyon, France). Using Curle's analogy they developed a simplified model based on the simultaneous measurement of the SP near the trailing edge to predict the far-field noise. In the same facility, Christophe et al [12] conducted measurements of a so-called Controlled Diffusion airfoil and proposed models based on LES calculations of the incompressible flow and either on Amiet's or Curle's theories for predicting the far-field acoustic pressure. These two experiments were conducted at relatively low Reynolds numbers $\left(R e \approx 1.5 \times 10^{5}\right)$ and therefore include flow features characteristics of bluff body aerodynamics with distinct vortex shedding phenomena. More recently, Schuele and Rossignol [13] studied aeroacoustic noise from a stalled DU-96-W-180 airfoil in the AWB wind tunnel in Braunschweig. They derived a model based on classical trailing edge noise theory $[15,16]$ and tuned for the stalled conditions. Subsequently and in order to study lower frequencies that could not be measured acoustically, this experiment was conducted again by Suryadi and Herr [14] in the same wind tunnel, but this time the study concentrated on SP measurements in stalled conditions. Both experiments were conducted at a Reynolds number equal to $1.2 \times 10^{6}$. Finally, there have also been several attempts to simulate stall noise using 
hybrid RANS/LES combined with acoustic analogies or related techniques [12, 17, 7]. However, these methods are still very demanding in terms of computational resources and cannot yet be applied in an engineering design context. Nevertheless, these can prove very valuable for understanding the mechanisms behind stall noise generation and to validate engineering models.

In the context of wind turbine noise in which this work has been undertaken, a number of studies related to stall noise have been conducted $[18,19,20]$. A recent analysis of wind turbine field measurements has shown that stall is most probably related to intermittent amplitude modulation effects [21]. Although the present study mainly focuses on wind turbine applications, its results may be in principle applied to any blade or airfoil stall case for which flow conditions do not significantly depart from the experimental conditions considered herein.

The aim of this paper is to study the turbulent characteristics of stall and to develop an engineering semi-empirical model for stall noise. Since the present study is performed in the context of wind turbines, relatively high Reynolds number flows (i.e. $1 \times 10^{6}<$ $R e<6 \times 10^{6}$ ) are considered. The analysis is based on a series of wind tunnel experiments as described in Section 2. Specific aerodynamic measurement data characterizing stall are briefly reported in Section 3. These are used later in the paper to identify stall and interpret additional measurement data. The turbulence characteristics of a stalled flow field useful for a noise model derivation, namely convection velocity, spatial correlations and SP spectra, are investigated in Sections 4, 5 and 6, respectively. The results of these analyses are used to develop a stall noise model in Section 7 which is validated against independent anechoic wind tunnel experiments in Section 8. Conclusions are drawn and possible improvements to the model are discussed in the last section.

\section{Description of Experiments}

The experimental data used in this article were acquired during several measurement campaigns.

Firstly, measurements of four different airfoil sections were conducted in the LM Wind Power wind tunnel in Lunderskov, Denmark [22]. This tunnel is a classical closed- 
loop aerodynamic wind tunnel with a $7 \mathrm{~m}$ closed test section. It has a $1.35 \mathrm{~m}$ wide and $2.7 \mathrm{~m}$ high cross-section specifically designed for the testing of wind turbine airfoils with little blockage and limited streamline curvature and a maximum wind speed of $105 \mathrm{~m} / \mathrm{s}$. The turbulence intensity measured in the clean wind tunnel configuration was estimated to be around $0.1 \%[23,24]$. All tested airfoil sections span the width of the tunnel and have a chord $C=0.9 \mathrm{~m}$. The shapes of the tested two-dimensional sections are the NACA-0015, NACA-63-418, RISØ-B1-18 and RISØ-C2-18 airfoil profiles, the three latter ones being used on wind turbines. The first airfoil has a $15 \%$ thickness relative to the chord while the remaining ones are $18 \%$ thick. Additional tests were also performed with a turbulence grid located at the inlet of the test section. This grid raised the inflow turbulence intensity to approximately 1.5\% [25]. The cross-sectional blockage ratio for the different airfoils tested in stalled conditions ranges from 10 to $12 \%$.

Secondly, a $0.6 \mathrm{~m}$ chord NACA-64-618 airfoil section was measured in the Virginia Tech Stability Wind Tunnel. The $7.3 \mathrm{~m}$ long and $1.83 \mathrm{~m} \times 1.83 \mathrm{~m}$ rectangular closed test section of this closed-loop tunnel is specifically designed with Kevlar walls, so that airfoils can be simultaneously measured aerodynamically and acoustically using a microphone array located outside the test section. Turbulence intensities of less than $0.05 \%$ were reported from measurements in the hard-walled test section configuration. More details about this wind tunnel specifications can be found in the literature $[26,9$, 27]. The blockage ratio for the maximum tested AoA is $9.7 \%$.

Note that even if measuring an airfoil in a wind tunnel does influence the stall characteristics, the purpose of the aerodynamic analysis performed in the following section is not to obtain qualitatively accurate aerodynamic data, but only to detect stall and separation location which is still possible regardless of wind tunnel effects.

The above wind tunnel measurements were conducted at various Reynolds numbers varying from $R e=1.0 \times 10^{6}$ to $6.0 \times 10^{6}$, with $R e=\rho U_{\infty} C / \mu$ where $\rho$ is the air density and $U_{\infty}$ the freestream velocity, and for varying AoAs with step increments of $2^{o}$. In some cases, the airfoils were also tested with zig-zag (ZZ) tapes in order to trigger transition. The tapes thickness is equal to $0.4 \mathrm{~mm}$ and their width between extremities 
in the flow direction is equal to $1 \mathrm{~cm}$. These were glued near the leading edge of the airfoils at $5 \%$ and $10 \%$ chord on the suction and pressure sides, respectively. Here and pressure measurements in small and awkward places as the probe is terminated with 
a small diameter tube. The probe itself was independently calibrated with the tubing device. The end of the probe tube was taped very close to the flushing adaptor hole blade span locations. The corresponding pressure transducers were also installed inside 


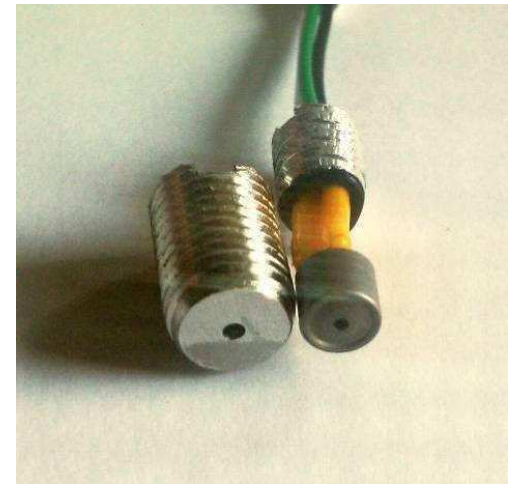

(a) KE 4-211-2 microphone and flushing adaptor
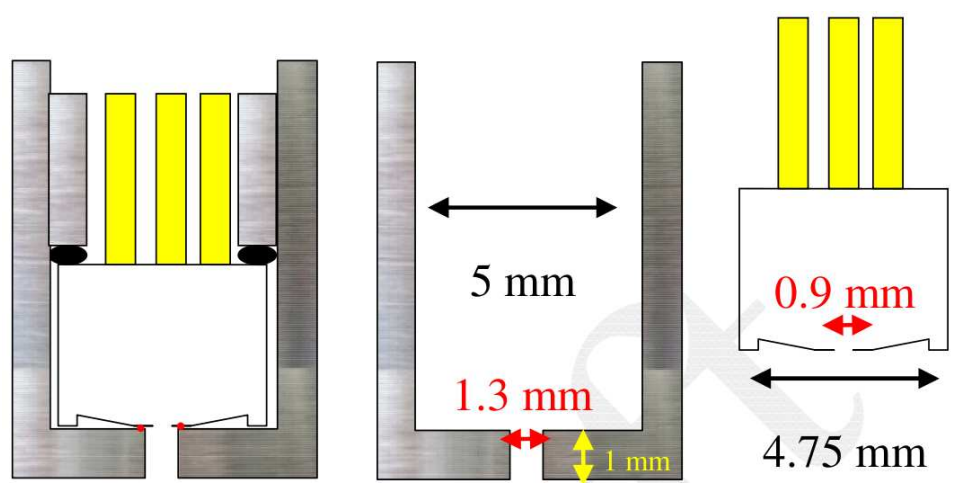

(b) Sketch of microphone mounting

Figure 2: Surface microphones set-up [Pictures courtesy of Brüel \& Kjær]

the blade. Atmospheric conditions were monitored with a nearby meteorology mast. Operational conditions such as rotor speed, power, etc. were also recorded. All the were not conducted (identified as $\mathrm{n} / \mathrm{a}$ in Table 1 ) or measurements failed for individual 


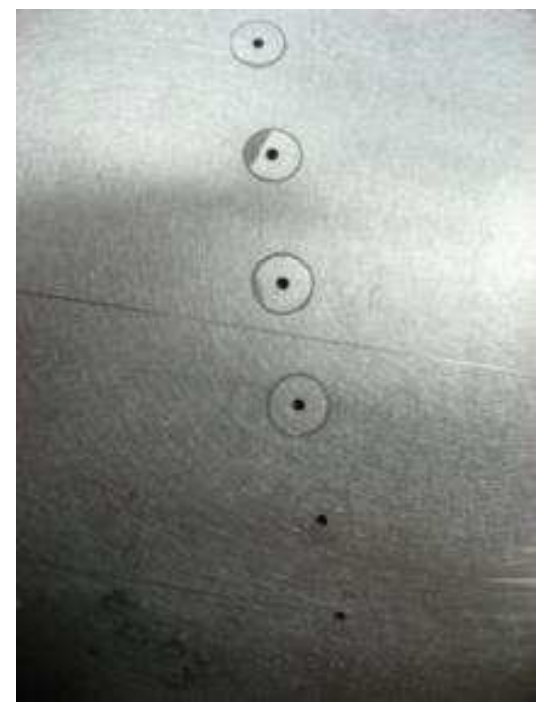

(a) Wind tunnel airfoil section

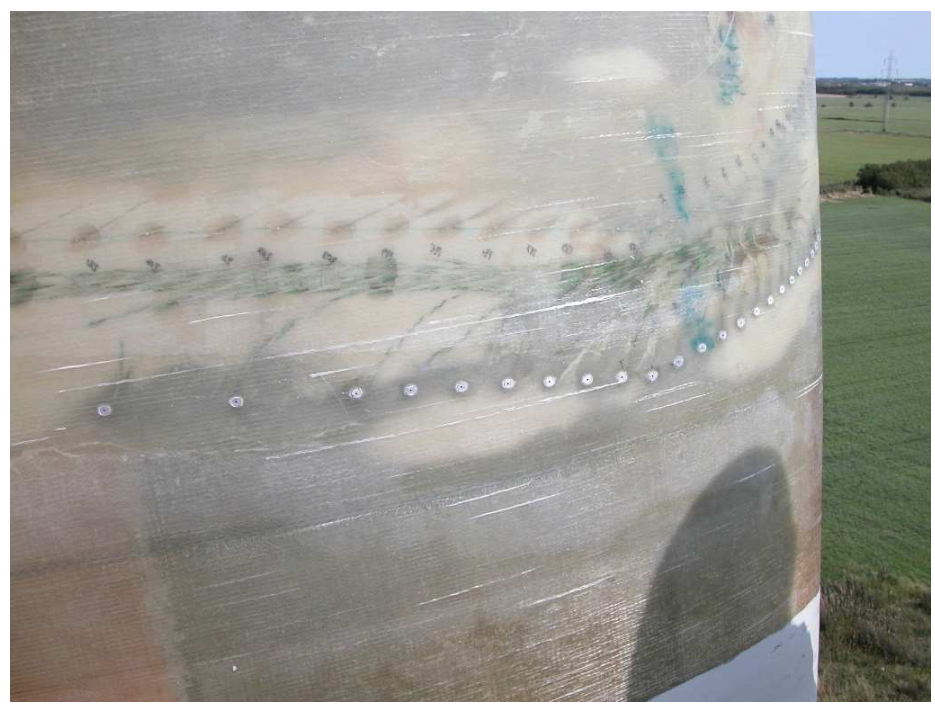

(b) LM 38.8 blade

Figure 3: Flush-mounted SP microphones

AoAs. For this reason and for the sake of brevity, measurements of the RIS $\varnothing$-C218 airfoil which is the most complete data set, will be more extensively investigated in this article. Measurement data of the remaining airfoil sections will be displayed occasionally in order to demonstrate the universality of our conclusions or possibly for pointing out particular phenomena. 


\begin{tabular}{|c|c|c|c|c|}
\hline Airfoil section \& tunnel & Reynolds number & Clean airfoil & ZZ-tape & Turbulence grid \\
\hline \multirow{3}{*}{$\begin{array}{l}\text { NACA-0015 } \\
\text { LM tunnel }\end{array}$} & \multirow{3}{*}{$\begin{array}{c}R e=1.8 M \\
R e=3 M \\
R e=3.6 M\end{array}$} & $\alpha=06-20^{\circ}$ & $\mathrm{n} / \mathrm{a}$ & $\mathrm{n} / \mathrm{a}$ \\
\hline & & $\alpha=06-20^{\circ}$ & $\mathrm{n} / \mathrm{a}$ & $\mathrm{n} / \mathrm{a}$ \\
\hline & & $\alpha=06-20^{\circ}$ & $\mathrm{n} / \mathrm{a}$ & $\mathrm{n} / \mathrm{a}$ \\
\hline \multirow{5}{*}{$\begin{array}{l}\text { RIS } \varnothing-B 1-18 \\
\text { LM tunnel }\end{array}$} & \multirow{5}{*}{$\begin{array}{c}R e=1.6 M \\
R e=3 M \\
R e=4 M \\
R e=5 M \\
R e=6 M\end{array}$} & $\alpha=06-20^{\circ}$ & $\alpha=06-20^{\circ}$ & $\alpha=06-20^{\circ}$ \\
\hline & & $\alpha=06-20^{\circ}$ & $\alpha=06-20^{\circ}$ & $\alpha=06-20^{\circ}$ \\
\hline & & $\alpha=06-20^{\circ}$ & $\mathrm{n} / \mathrm{a}$ & $\mathrm{n} / \mathrm{a}$ \\
\hline & & $\alpha=06-20^{\circ}$ & $\mathrm{n} / \mathrm{a}$ & $\mathrm{n} / \mathrm{a}$ \\
\hline & & $\alpha=06-20^{\circ}$ & $\alpha=06-20^{\circ}$ & $\alpha=06-18^{\circ}$ \\
\hline \multirow{5}{*}{$\begin{array}{l}\text { RIS } \varnothing-C 2-18 \\
\text { LM tunnel }\end{array}$} & \multirow{5}{*}{$\begin{array}{c}R e=1.6 M \\
R e=3 M \\
R e=4 M \\
R e=5 M \\
R e=6 M\end{array}$} & $\alpha=06-16^{\circ}$ & $\alpha=06-16^{\circ}$ & $\alpha=06-16^{\circ}$ \\
\hline & & $\alpha=06-16^{\circ}$ & $\alpha=06-16^{\circ}$ & $\alpha=06-16^{\circ}$ \\
\hline & & $\alpha=06-16^{o}$ & $\mathrm{n} / \mathrm{a}$ & $\mathrm{n} / \mathrm{a}$ \\
\hline & & $\alpha=06-16^{\circ}$ & $\mathrm{n} / \mathrm{a}$ & $\mathrm{n} / \mathrm{a}$ \\
\hline & & $\alpha=06-16^{\circ}$ & $\alpha=06-16^{\circ}$ & $\alpha=06-16^{\circ}$ \\
\hline \multirow{5}{*}{$\begin{array}{c}\text { NACA-63-418 } \\
\text { LM tunnel }\end{array}$} & \multirow{5}{*}{$\begin{array}{c}R e=1.6 M \\
R e=3 M \\
R e=4 M \\
R e=5 M \\
R e=6 M\end{array}$} & $\alpha=06-16^{\circ}$ & $\mathrm{n} / \mathrm{a}$ & $\alpha=06-16^{\circ}$ \\
\hline & & $\alpha=06-16^{\circ}$ & $\alpha=06-16^{\circ}$ & $\alpha=06-16^{\circ}$ \\
\hline & & $\alpha=06-16^{\circ}$ & $\alpha=06-16^{\circ}$ & $\alpha=06-16^{\circ}$ \\
\hline & & $\alpha=06-16^{\circ}$ & $\alpha=06-16^{\circ}$ & $\alpha=06-16^{\circ}$ \\
\hline & & $\alpha=06-16^{\circ}$ & $\alpha=06-16^{\circ}$ & $\mathrm{n} / \mathrm{a}$ \\
\hline \multirow{3}{*}{$\begin{array}{c}\text { NACA-64-618 } \\
\text { Virginia Tech tunnel }\end{array}$} & \multirow{3}{*}{$\begin{array}{l}R e=1.0 M \\
R e=1.5 M \\
R e=1.9 M\end{array}$} & $\alpha=06-14^{\circ}$ & $\mathrm{n} / \mathrm{a}$ & $\mathrm{n} / \mathrm{a}$ \\
\hline & & $\alpha=06-14^{o}$ & $\alpha=06-14^{\circ}$ & $\mathrm{n} / \mathrm{a}$ \\
\hline & & $\alpha=06-14^{o}$ & $\alpha=06-14^{\circ}$ & $\mathrm{n} / \mathrm{a}$ \\
\hline
\end{tabular}

Table 1: Test matrix of measurements (n/a: experimental data not available) 

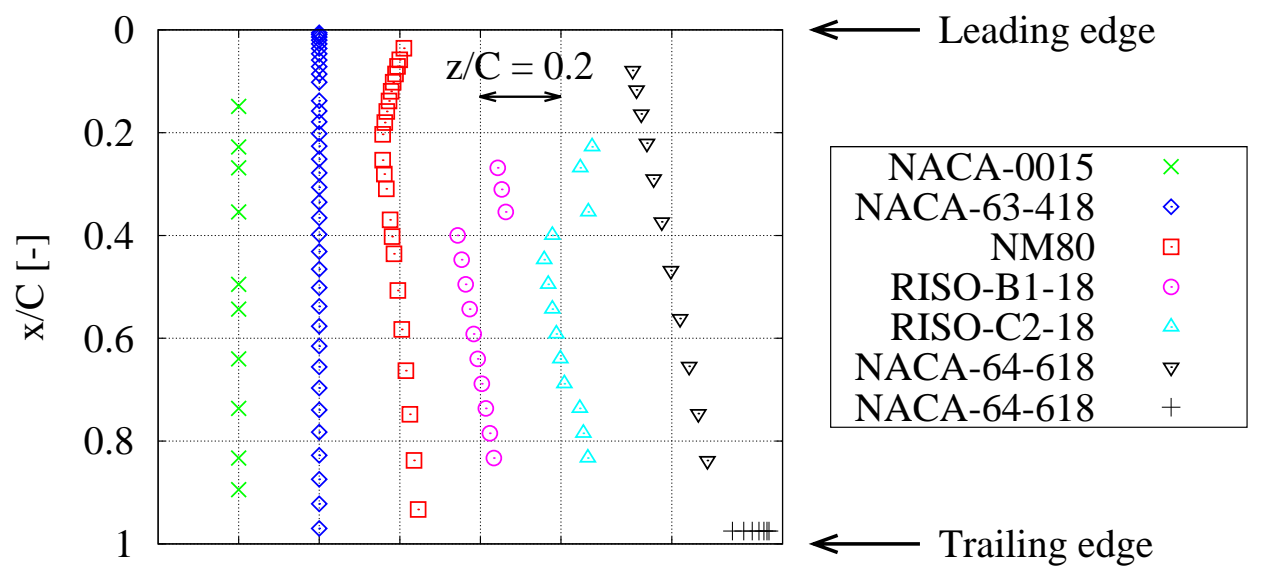

Figure 4: SP microphone distributions on the suction side of the different airfoil sections 


\section{Airfoil Aerodynamic Properties and Stall}

As mentioned in the introduction, stall is associated with a decrease of lift. In Fig. 5(a), the lift coefficient as a function of the AoA $\alpha$, or polar curve, is plotted for the clean RISØ-C2-18 airfoil at all measured Reynolds numbers. It can be observed that up to a certain AoA, lift grows linearly. Then, the slope of these curves starts to decrease indicating the initiation of stall, in conjunction with the emergence of a recirculating flow near the trailing edge (see analysis of pressure coefficient distributions below). Soon after, the lift itself decreases indicating the rapid growth of the separated region to larger portions of the airfoil chord. The existence of a hysteresis effect can clearly be seen in the figure. Indeed, as the airfoil lift was measured both for increasing and decreasing AoA, the two associated curves differ around $\alpha=12^{\circ}$ where stall occurs and this phenomenon amplifies as the Reynolds number increases.

The effect of the ZZ tape and of the turbulence grid on the airfoil polars is investigated in Figs. 5(b) and (c), respectively. Only two Reynolds numbers $R e=1.6 \times 10^{6}$ and $6 \times 10^{6}$ are displayed. For reference, the polars for the clean airfoil are also displayed. It can be seen that both ZZ tape and turbulence grid have a tendency to decrease the lift slope at lower AoAs, and causing earlier stall in the former case while delaying stall in the latter one, irrespectively of the Reynolds number. The hysteresis effect noticed for the clean airfoil is also observed.

The above scenarios can be investigated in more detail by looking at the pressure coefficient distributions around the RIS $\varnothing-C 2-18$ airfoil as displayed in Figs. 6(a-b-c) for the clean airfoil, with ZZ tape and with turbulence grid, respectively. Note that the pressure $p$ measured by the pressure taps is plotted as a differential pressure relatively to the freestream pressure $p_{\infty}$ and is non-dimensionalized with the freestream dynamic pressure yielding the pressure coefficient defined as $C_{p}=\left(p_{\infty}-p\right) /\left(0.5 \rho U_{\infty}^{2}\right)$. This coefficient is further averaged in time in order to get stationary values. These $C_{p}$ distributions are displayed at various AoAs and Reynolds numbers. In the case of the clean airfoil, $C_{p}$ distributions are plotted at $\alpha=10^{\circ}$ for which the flow is attached in order to emphasize the differences with the stalled flow. At $\alpha=12^{\circ}$, flow separation 


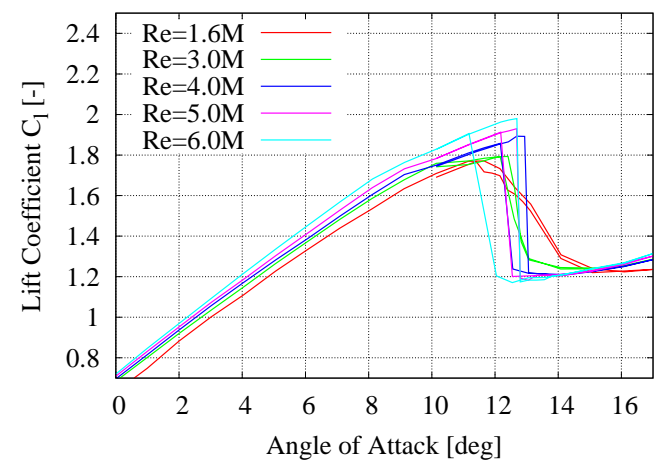

(a) Clean airfoil

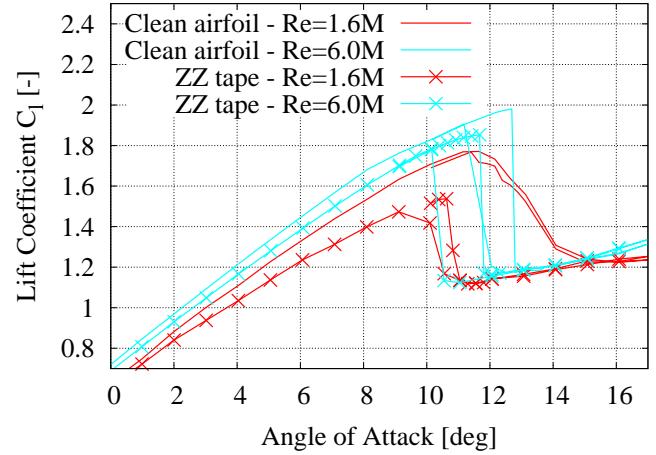

(b) ZZ tape

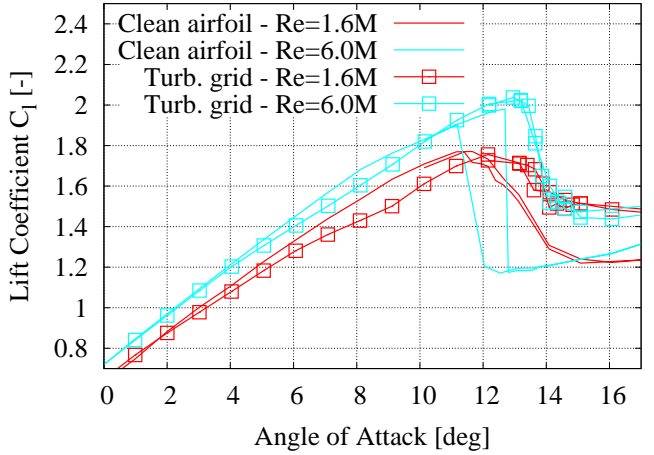

(c) Turbulence grid

Figure 5: RIS Ø-C2-18 airfoil - Polar curves

occurs for $R e=6 \times 10^{6}$ but the flow remains attached for lower Reynolds numbers. In contrast to attached flow conditions, the $C_{p}$ distribution exhibits a plateau on the suction side characteristic of stall separation. For higher AoAs, the flow is stalled at all Reynolds numbers. Similar behaviour can be observed in the case of the tripped airfoil and with turbulence grid. However, it can be seen that the $\mathrm{ZZ}$ tape is causing earlier stall at $R e=1.6 \times 10^{6}$ and $\alpha=12^{\circ}$ whereas the turbulence grid is postponing stall at $R e=6 \times 10^{6}$ and $\alpha=12^{\circ}$. The behavior of the pressure coefficient described above are in agreement with the observations made for the polar curves. Note that the $C_{p}$ plateaux observed near the leading edge does not mean that the flow separates, but are a consequence of the pressure recovery following the leading edge pressure surge specific to this airfoil shape design.

It should mentioned here that when the airfoil is stalled, the small chord interval where the pressure coefficient as a function of the chord abscissa is transitioning from 
a decreasing to a flat curve is a good estimator of the separation point location. In 255 the cases the pressure distributions are available, this estimation will be used in our modelling approach later in this paper.

Similar conclusions regarding the above described stall features can be drawn from the remaining airfoils data. 

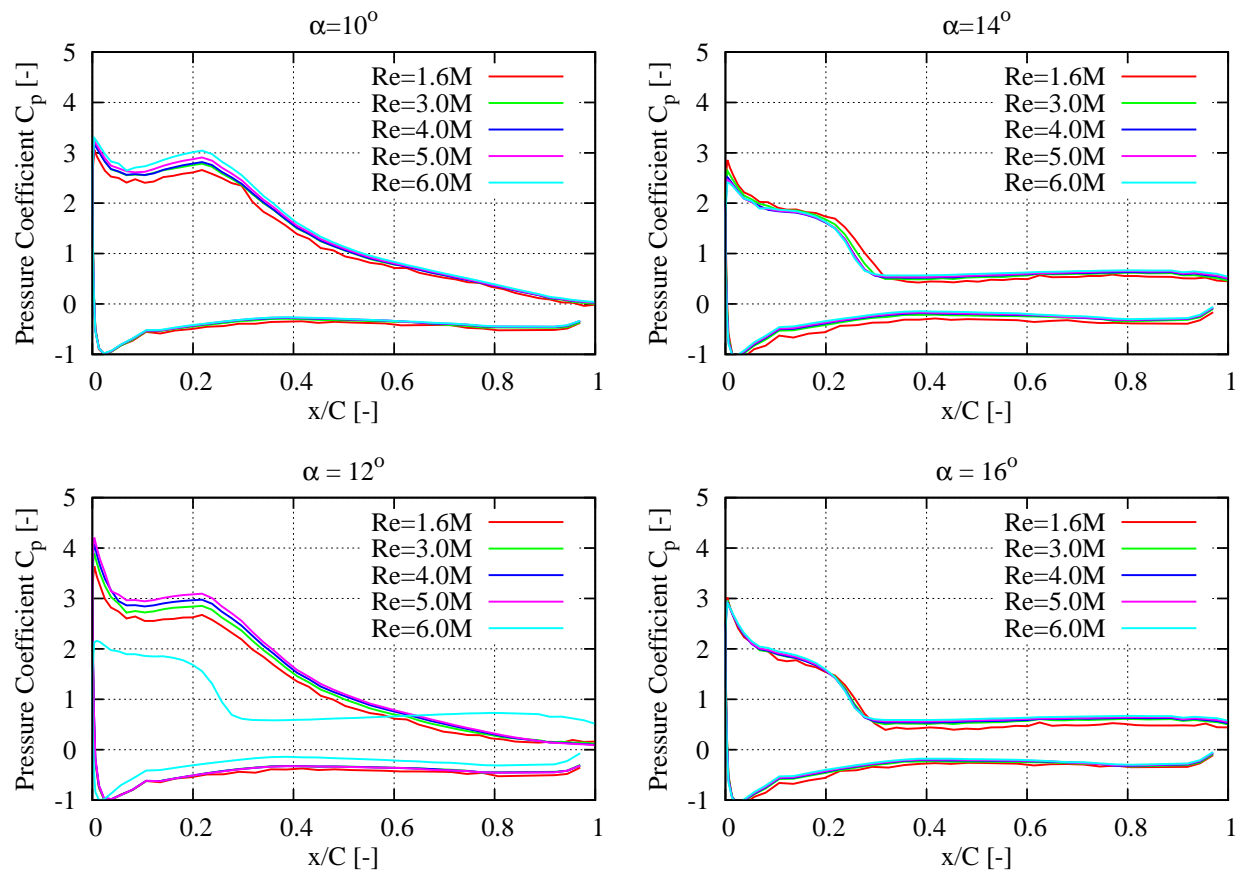

(a) Clean airfoil
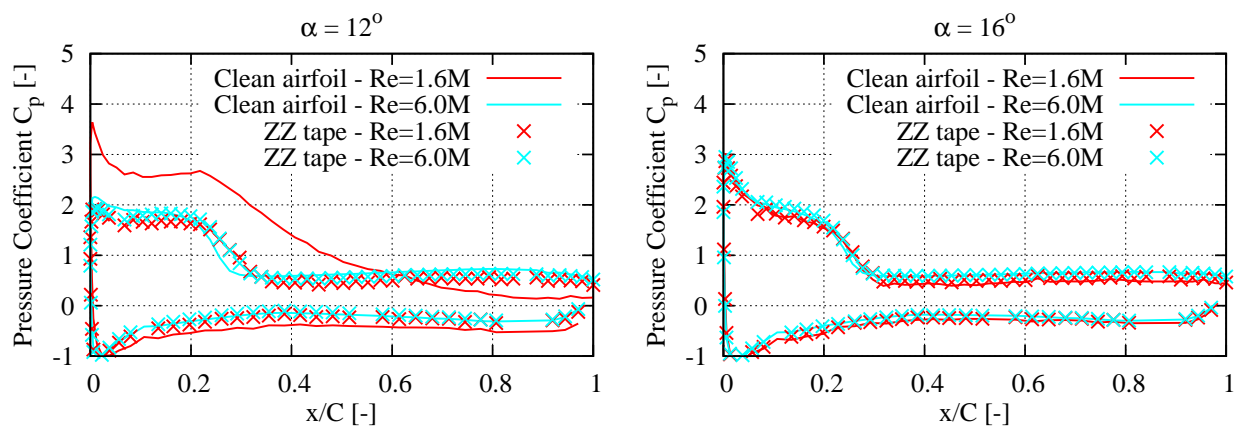

(b) ZZ tape
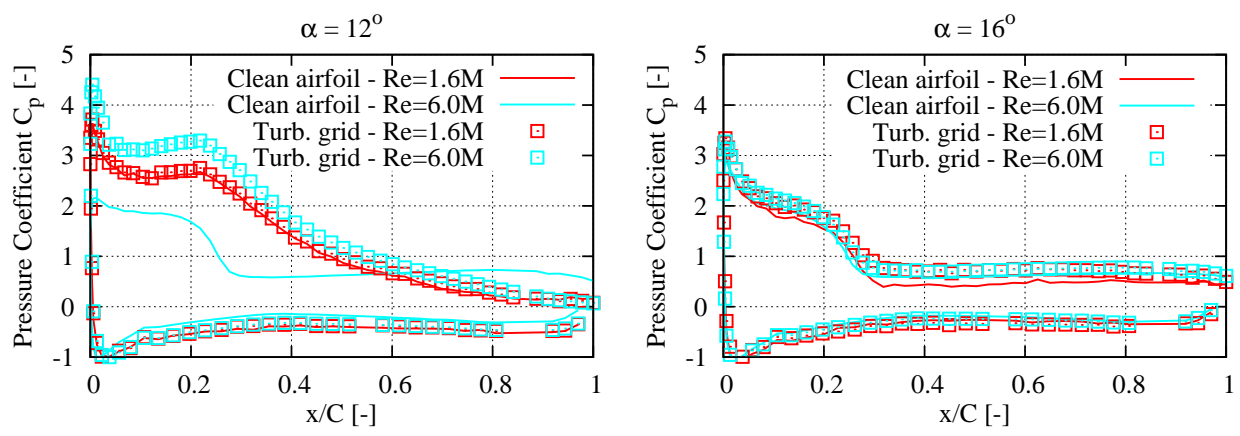

(c) Turbulence grid

Figure 6: RISØ-C2-18 airfoil - Pressure coefficient distributions 


\section{Convection Velocity in Stall and its Scaling}

Both within the attached turbulent boundary layer developing along an airfoil surface and the separated flow region of a stalled airfoil, turbulent vortical structures are generated and convect downstream. In the LES simulation performed by Christophe et al [12] and in the case of a stalled flow, the vortices are observed rolling over the airfoil suction side and grazing at the trailing edge until they are eventually convected away into the wake. The averaged velocity at which turbulent vortices convect, 'convection velocity' for short, can be evaluated using pairs of microphones [36, 37]. One method consists in using the signals from two SP microphones separated by a distance $\Delta x$ along the chord and calculating the cross-correlation function between the two time-series as:

$$
\rho_{x-x_{\mathrm{ref}}}(\Delta \tau)=<p\left(x_{\mathrm{ref}}, t+\Delta \tau\right) p(x, t)>
$$

where the angle brackets $\langle\ldots\rangle$ denote ensemble averaging, $x_{\text {ref }}$ the reference microphone position where the convection velocity is evaluated, and $x$ a microphone located upstream with $\Delta x=x_{\text {ref }}-x$ (see chosen illustrative cases in Fig. 9 and discussion later in this section). The time interval $\Delta \tau_{\max }$ at which this function reaches its maximum value is estimated. The SP measured at the upstream microphone and generated by specific vortical structures will be most correlated with the SP measured at the reference microphone and generated by the same structures as these have convected to this latter location. Therefore, the convection velocity can be estimated by the simple formula:

$$
U_{\mathrm{cv}}=\Delta x / \Delta \tau_{\max }
$$

Note that this quantity is not characterizing the speed of each individual turbulent vortex, but rather the speed at which their collective footprint as SP fluctuations travel.

The computed convection velocities non-dimensionalized by the freestream velocity are plotted for the clean RISØ-C2-18 airfoil, with ZZ tape and with turbulence grid in Figs. 7(a-b-c), respectively. These correspond to the cases displayed in Figs. 6(ab-c), allowing to identify where the flow is attached or separated. Where the flow is attached, the convection velocities clearly take values closer to $U_{\mathrm{cv}} / U_{\infty}=1$ than for the 
stalled cases. The convection velocity decreases as the trailing edge is approached. This illustrates the action of the adverse pressure gradient and the deceleration of the flow along the airfoil chord which eventually results in separation for high AoAs. Where the flow is separated, the normalized convection velocity becomes relatively constant along the airfoil chord and takes values between 0.5 and 0.6. The chord locations where the convection velocity becomes constant is an estimator of the separation location. It can be checked that these locations roughly coincide with those that can be deduced from the pressure coefficient distributions in Fig. 6 as explained in Section 3.

The convection velocities are plotted for two additional noteworthy cases. The first one is the NACA-64-618 airfoil in Fig. 8(a). The transition from attached flow in the leading edge region toward a separated flow closer to the trailing edge is clearly visible at $\alpha=14^{\circ}$. Note that the convection velocity in the attached flow region both at $\alpha=8^{\circ}$ and $14^{\circ}$ takes higher values than the freestream velocity as a result of the acceleration of the fluid flow as it convects around the airfoil leading edge.

In Fig. 8(b), the convection velocities calculated for the NACA-63-418 airfoil measured in the wind tunnel at $R e=5 \times 10^{6}$ are compared to those deduced from the measurements on the blade of the NM80 wind turbine at two AoAs: for attached flow condition at $\alpha=6^{\circ}$ and stalled condition at $\alpha=14^{\circ}$. It can be observed that the curves are discontinuous. Individual reference microphones have been left out if the convection velocities computed at these locations take extreme non-physical values (i.e. larger than 1.5 or negative, see analyses of cross-correlation functions and phase spectra below for clarification). Nevertheless, there exists a relative good agreement between the displayed wind tunnel and wind turbine results. Wind turbine results indicate a separation location closer to those from the wind tunnel with turbulent grid as expected.

310 In view of the previous results, the ratio of the convection velocity to the freestream velocity will be assumed constant in the separated flow region in the remaining of this article. The convection velocity is modelled as:

$$
U_{\mathrm{cv}}=0.55 \cdot U_{\infty}
$$

as an averaged value of the different airfoil measurements. It should be mentioned 
that a similar value of 0.6 was reported by Moreau et al [38] at low frequencies when frequencies (up to $500 \mathrm{~Hz}$ ) in accordance with the results of Moreau et al [38]. In the case of the NM80 measurements, only some of the closest pairs of microphones exhibit a linear slope. The valid frequency limit varies from $150 \mathrm{~Hz}$ for the reference microphone 
at $x_{\text {ref }} / C=84 \%$ to $500 \mathrm{~Hz}$ for $x_{\text {ref }} / C=75 \%$. The phase errors mentioned earlier are 345 clearly visible in the respective cases. 

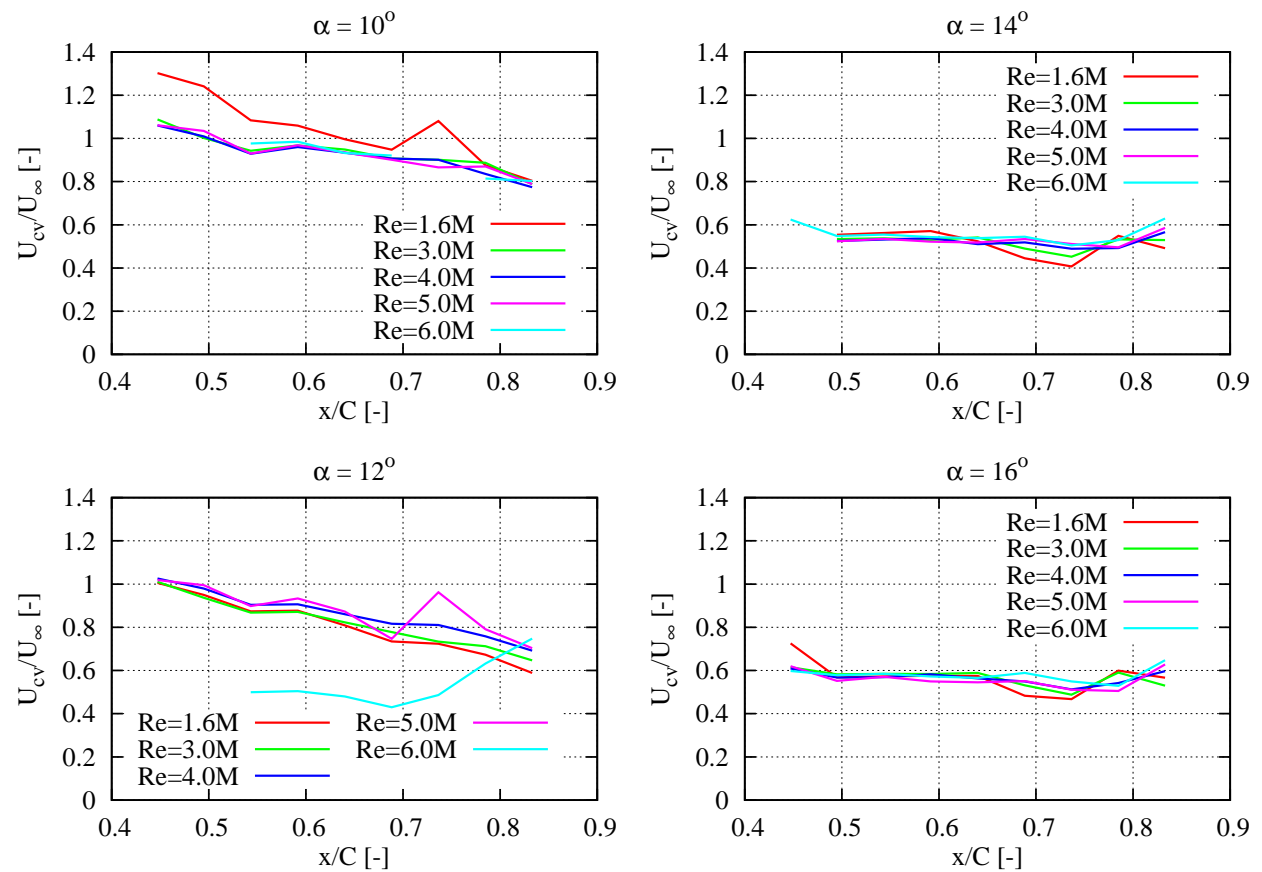

(a) Clean airfoil
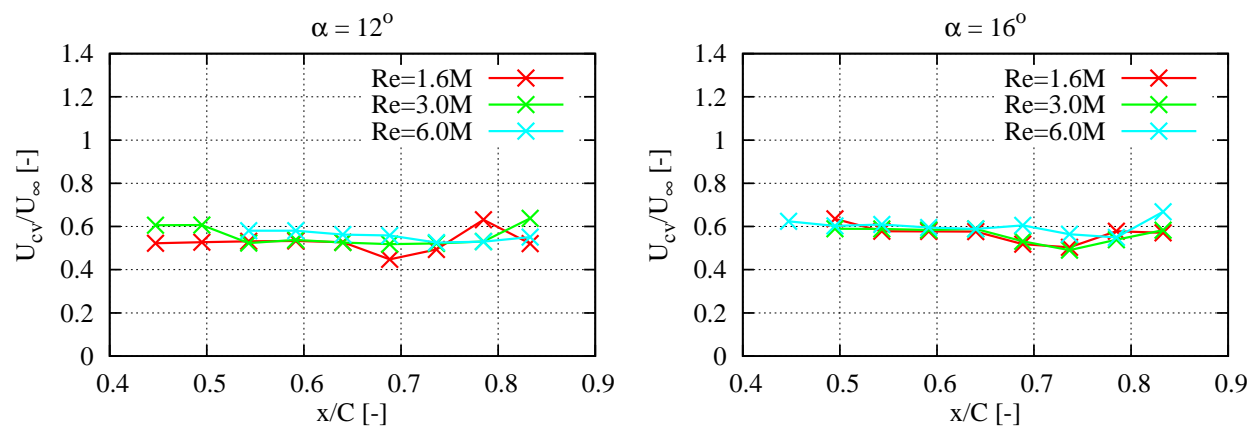

(b) ZZ tape
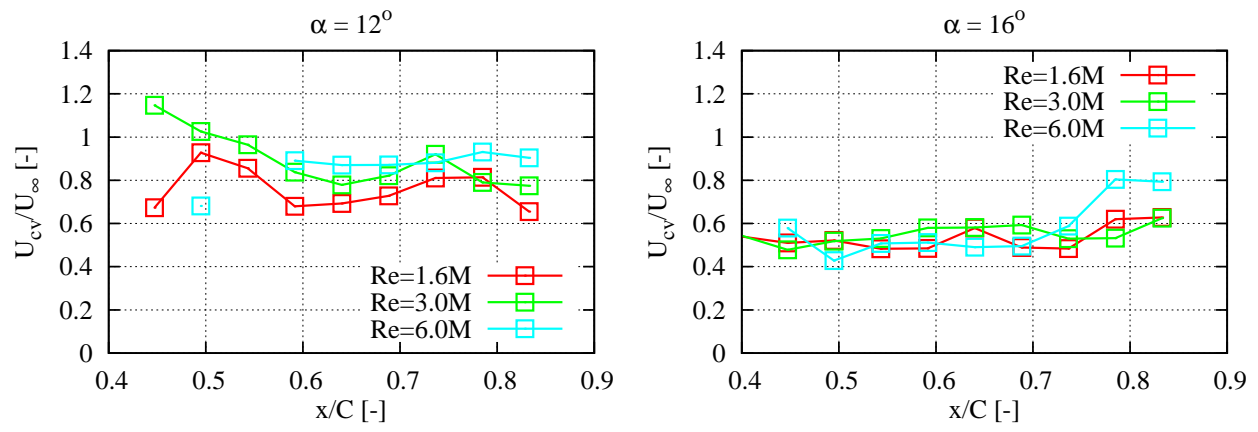

(c) Turbulence grid

Figure 7: RISØ-C2-18 airfoil - Convection velocity 

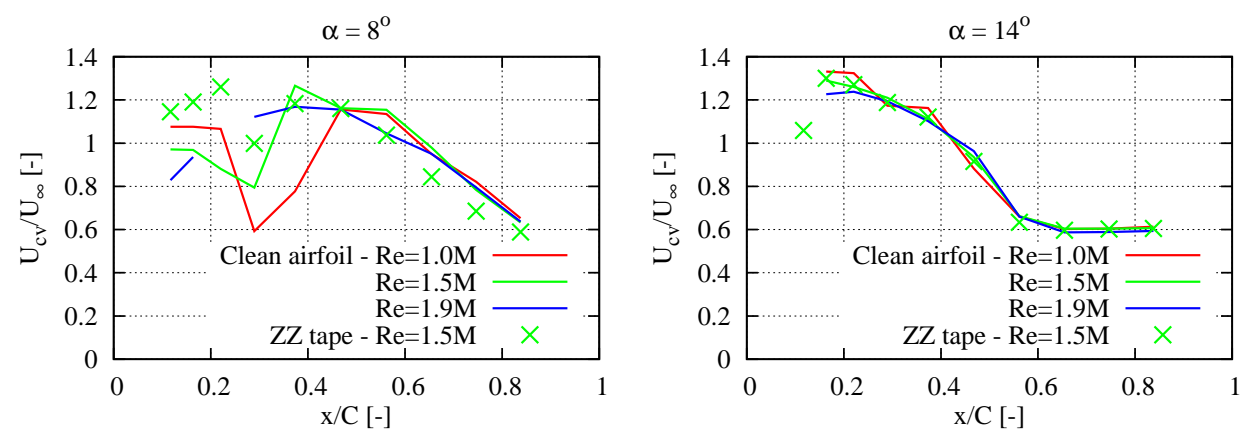

(a) NACA-64-618 airfoil
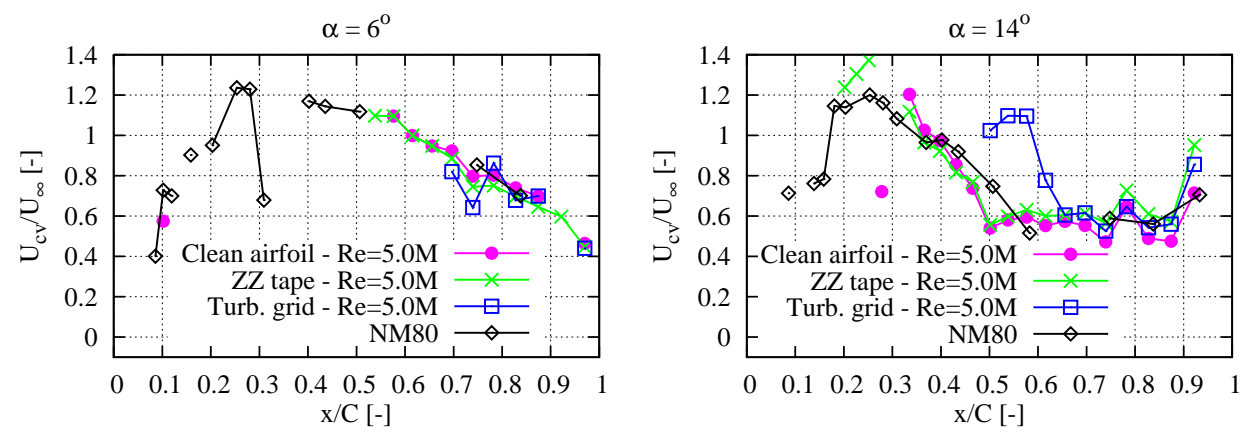

(b) NACA-63-418 airfoil and NM80 wind turbine

Figure 8: Convection velocity for NACA-64-618, NACA-63-418 and NM80 turbine 

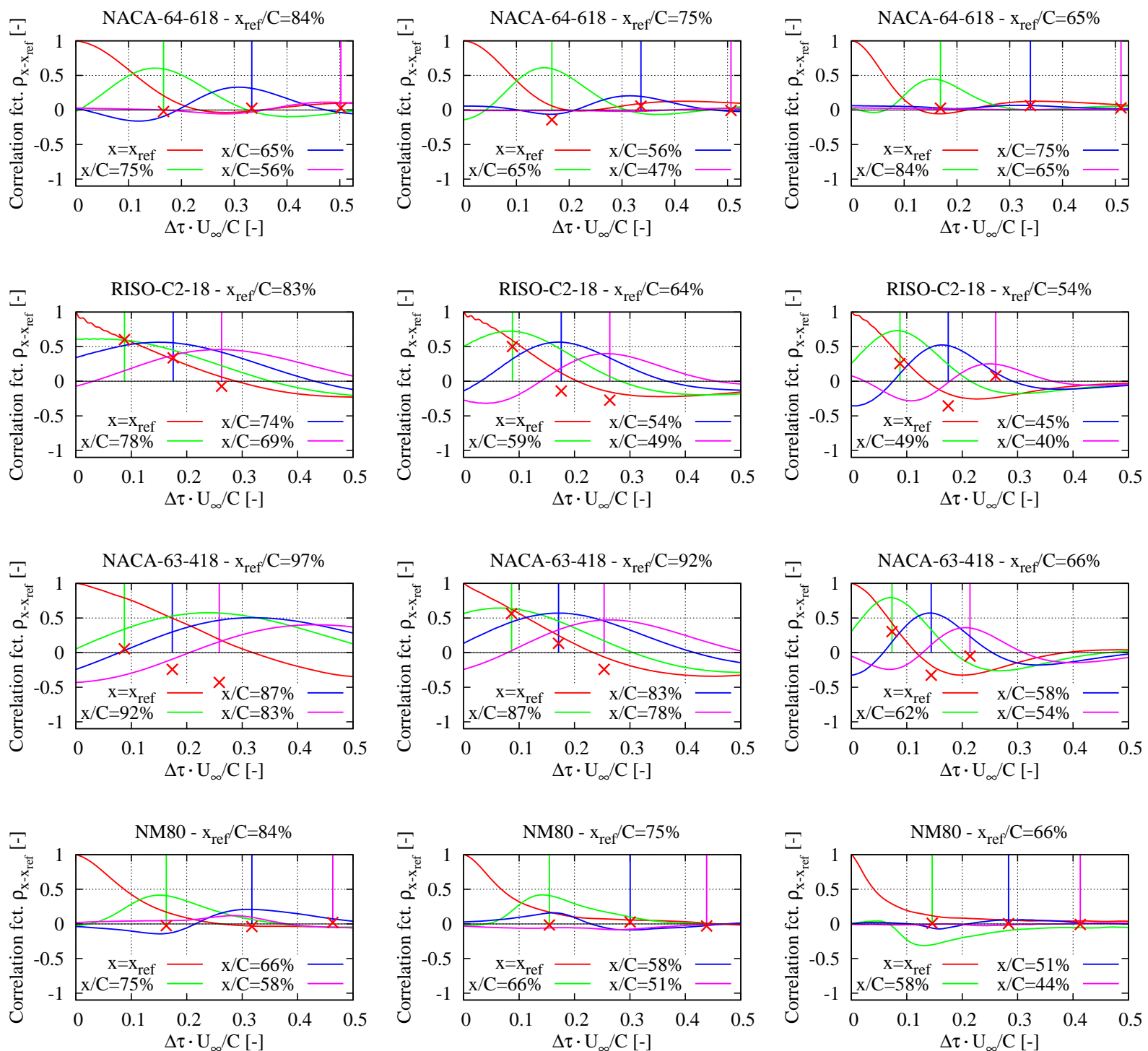

Figure 9: Cross-correlation function between various microphones along airfoil chord (Row \#1: NACA-64-6418 airfoil at $\alpha=14^{\circ}$ and $R e=1.5 \times 10^{6}$; Row \#2: RIS $\varnothing-C 2-18$ airfoil at $\alpha=16^{\circ}$ and $R e=6 \times 10^{6}$; Row \#3: NACA-63-418 airfoil at $\alpha=14^{\circ}$ and $R e=5 \times 10^{6}$; Row \#4: NM80 blade section at $\alpha=14^{\circ}$ and $R e=5 \times 10^{6}$; Vertical lines indicates $\Delta \tau_{\mathrm{cv}}=\Delta x /\left(0.55 \cdot U_{\infty}\right) ;$ Crosses at coordinates $\left\{\Delta \tau_{\mathrm{cv}}, \rho_{x-x_{\mathrm{ref}}}(\Delta \tau \equiv 0)\right\}$ 

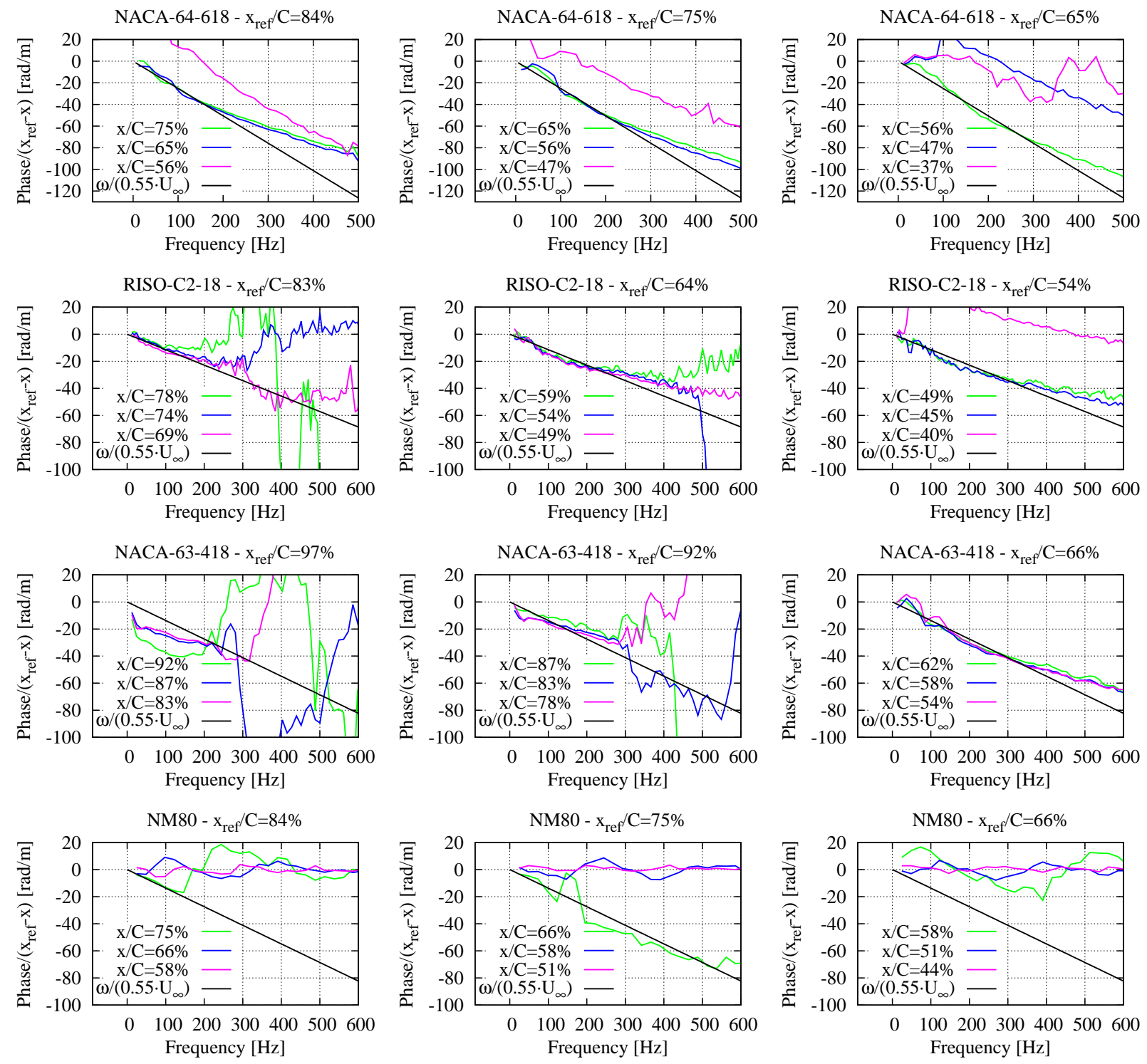

Figure 10: Phase between various microphones along airfoil chord (Same cases as in Fig. 9) 


\section{Characterization of Stall through Spatial Correlation}

An important attribute defining a turbulent flow field is its spatial correlation characteristics. In our case, the turbulent quantity of interest is the SP and we are interested in its correlation both in the chord and span directions. Microphones were distributed along the span of the airfoil section only during the experimental campaign conducted in the Virginia Tech wind tunnel (see Fig. 4). For all other measurements performed in the LM wind tunnel and on the NM80 turbine, microphones were only distributed along the chord. Therefore, the development of a model for the spanwise correlation is based on the Virginial Tech measurement data only. It is assumed that this model can be generalized to more general airfoil types and Reynolds numbers.

\subsection{Correlation Length in Chord Direction and its Scaling}

The correlation length in the chord direction can be estimated using a single microphone. Frozen turbulence is assumed together with the fact that the turbulent fluctuations convect parallel to the airfoil chord according to Eq. (1). Hence, the temporal auto-correlation function of the SP time-series $p(x, t)$ recorded at time $t$ by one microphone located at the chord abscissa $x$ is used to estimate the spatial auto-correlation function as a function of the separation length $\xi$ :

$$
<p(x, t) p(x+\xi, t)>\equiv<p(x, t) p(x, t-\tau)>
$$

where the ensemble averaging can be achieved by assuming temporal homogeneity, calculating the temporal auto-correlation functions centered at various instants $t$ during the time-series, and averaging the resulting functions. Here, $\tau$ is the separation time of the temporal auto-correlation function and is equal to $\tau \equiv \xi / U_{\mathrm{cv}}$ due to the frozen turbulence assumption. The correlation length $L_{x}$ is then defined as:

$$
L_{x}(x)=\int_{0}^{\infty} \frac{<p(x, t) p(x+\xi, t)>}{<p(x, t) p(x, t)>} \mathrm{d} \xi=U_{\mathrm{cv}} \cdot \int_{0}^{\infty} \frac{<p(x, t) p(x, t-\tau)>}{<p(x, t) p(x, t)>} \mathrm{d} \tau
$$

In practice, the computation of $L_{x}$ makes use of the Wiener-Khinchin theorem and the FFT algorithm [39]. The convection velocity is computed using Eq. (1). 
The calculated correlation lengths along the chord of the RIS $\varnothing-\mathrm{C} 2-18$ airfoil are displayed in Figs. 11(a-b-c) for the clean airfoil, with ZZ tape and with turbulence grid, respectively. Both the chord abscissa $x$ and the correlation lengths $L_{x}$ have been non-dimensionalized by the airfoil chord $C$. More importantly, the non-dimensionalized correlation length have been scaled by a factor $R e_{M}^{-1 / 2}$. The 'reduced' Reynolds number $R e_{M}=R e / 10^{6}$ is used here and in the remaining of this article in order to avoid very large or very small ranges for the various plot axes. This particular scaling is chosen as it is found to be the best choice in order for the slopes of the correlation length curves as a function of the chord abscissa to collapse, momentarily disregarding the case $R e=$ $1.6 \times 10^{6}$ which clearly deviates from the results at higher Reynolds numbers (this issue is addressed later). When stall conditions are met, the correlation length grows roughly linearly as a function of the distance from the separation point. It is reminded here that the separation point can be inferred from the pressure coefficient distribution or the convection velocity plots, as explained in Sections 3 and 4, respectively. Fitting lines to the part of the correlation length curves where they behave linearly and locating where these lines intersect the $x / C$-axis show that these locations approximately coincide with the separation points that can be deduced from Figs. 6(a-b-c). Note that the calculated correlation lengths behave erratically in the vicinity of the separation point, however it does not compromize the curve fitting (see below) as this remains localized. Approaching the trailing edge, the correlation length has a tendency to deviate from its linear behaviour and its slope progressively decreases. This may be slightly attenuated if using the measured convection velocities, which was observed sometimes increasing toward the trailing edge in Section 4, for computing the correlation length. However, this could not be done systematically because of the observed phase errors and the occasional lack of numerical estimation for the convection velocity. For the considered Reynolds numbers (i.e. again disregarding the case $R e=1.6 \times 10^{6}$ ), the slopes of the linear part of these curves approach a nearly common value so that:

$$
\frac{L_{x}^{+}(x)}{C} \approx 0.4 \cdot \frac{x-x_{\mathrm{sep}}}{C} \cdot R e_{M}^{-1 / 2}
$$

where the separation point location $x=x_{\text {sep }}$ is specific to each case (i.e. AoA and 
Reynolds number) and defines where the fitted linear curve intersects the chord axis. The superscript ${ }^{+}$denotes the fact that the above relationship is suited for high Reynolds

as well as measurements with turbulence grid at $R e=3 \times 10^{6}$, exhibit significant lower slopes compared to the remaining cases (see below for further details). This is somehow also true for the clean airfoil at $\alpha=16^{\circ}$ and $R e=3 \times 10^{6}$.

The case of the NACA-63-418 airfoil and measurements on the NM80 wind turbine blade are investigated in Fig. 12. The separation points that can be deduced from line fitting do not precisely correspond to those that were deduced from the convection velocity curves in Fig. 8(b). The latter predicted separation points lie slightly downstream (at $x / C=0.5$ ) of what can be deduced from the present curves $(x / C \approx 0.4)$. The separation point locations that can be predicted from the pressure coefficient distributions (see Fig. 18(a) further down) lie somewhere in between $(x / C \approx 0.45)$. These discrepancies are attributed to the various simplifications used to determine the correlation length. It is also noteworthy that the slopes of the correlation length curves as a function of the chord are slightly lower than in the case of the RIS $\varnothing-\mathrm{C} 2-18$, with an approximate slope factor of 0.35. Measurements on the NM80 turbine at a section corresponding to the same NACA-63-418 airfoil exhibit the same slightly lower slope. Therefore, the 0.4 slope factor might not be universal and small airfoil dependent variations may exist.

The following figures are a collection of results in the form of scatter plots. For each of the considered airfoils, all calculated correlation length curves are plotted, irrespectively of the AoA, Reynolds number or configuration, on the condition that the flow has stalled (i.e. for high enough AoAs). In these figures, the color convention differentiates the different Reynolds numbers and the different symbols refer to a clean, tripped or turbulence grid case. The abscissa has been shifted so that the origin corresponds to the separation point location $x=x_{\text {sep }}$. Figs. 13(a-b-c-d) display results for the RIS $\varnothing-C 2-18$, RISØ-B1-18, NACA-0015 and NACA-63-418 airfoils, respectively. The main additional finding is that the RIS $\varnothing-\mathrm{B} 1-18$ and NACA-0015 airfoils exhibit a 0.4 slope factor in agreement with the RIS $\varnothing-\mathrm{C} 2-18$ airfoil.

As observed above, the correlation lengths measured at $R e=1.6 \times 10^{6}$, and to some 
degree at $R e=3 \times 10^{6}$, do not follow the above $R e_{M}^{-1 / 2}$ scaling. It is found that the following scaling is more appropriate:

$$
\frac{L_{x}^{-}(x)}{C} \approx 0.13 \cdot \frac{x-x_{\mathrm{sep}}}{C} \cdot R e_{M}^{+1 / 4}
$$

$$
L_{x}(x)=c_{R}\left(R e_{M}\right) \cdot L_{x}^{-}(x)+\left(1-c_{R}\left(R e_{M}\right)\right) \cdot L_{x}^{+}(x)
$$

where the blending function $c_{R}\left(R e_{M}\right)$ is defined as:

$$
c_{R}\left(R e_{M}\right)= \begin{cases}1 & \text { for } R e_{M}<2, \\ 3-R e_{M} & \text { for } 2<R e_{M}<3 \\ 0 & \text { for } R e_{M}>3 .\end{cases}
$$

Finally, a survey of the estimated separation locations, as well as the measured and modelled correlation lengths at the most downstream microphone on the RIS $\varnothing-\mathrm{C} 2-18$ airfoil is provided in Table 2 . 


\begin{tabular}{|c|c|c|c|c|c|c|c|c|c|}
\hline$R e$ & \multicolumn{3}{|c|}{ Clean airfoil } & \multicolumn{3}{|c|}{ ZZ-tape } & \multicolumn{3}{|c|}{ Turbulence grid } \\
\hline & $12^{\circ}$ & $14^{\circ}$ & $16^{\circ}$ & $12^{\circ}$ & $14^{\circ}$ & $16^{\circ}$ & $12^{\circ}$ & $14^{\circ}$ & $16^{\circ}$ \\
\hline $1.6 M$ & $\star$ & $31.5 ; 6.16 ; 6.82$ & $32.0 ; 5.51 ; 6.75$ & $34.0 ; 5.61$ & $32.0 ; 5.95$ & $31.5 ; 5.70$ & $\star$ & $33.0 ; 4.44$ & $28.5 ; 5.77$ \\
\hline $3.0 M$ & $\star$ & $29.0 ; 8.65 ; 11.3$ & $29.0 ; 8.29 ; 11.3$ & $33.0 ; 7.30$ & $32.0 ; 7.67$ & $29.5 ; 8.08$ & $\star$ & $\star$ & $0.32 ; 6.75$ \\
\hline $4.0 M$ & $\star$ & $29.0 ; 8.36 ; 9.78$ & $28.5 ; 8.36 ; 9.86$ & \multicolumn{3}{|c|}{$\mathrm{n} / \mathrm{a}$} & \multicolumn{3}{|c|}{$\mathrm{n} / \mathrm{a}$} \\
\hline $5.0 M$ & $\star$ & $29.0 ; 7.30 ; 8.75$ & $28.5 ; 7.68 ; 8.82$ & \multicolumn{3}{|c|}{$\mathrm{n} / \mathrm{a}$} & \multicolumn{3}{|c|}{$\mathrm{n} / \mathrm{a}$} \\
\hline $6.0 M$ & $28.0 ; 4.64 ; 8.13$ & $28.5 ; 5.57 ; 8.05$ & $27.5 ; 6.03 ; 8.20$ & $31.0 ; 5.38$ & $30.5 ; 5.51$ & $28.5 ; 5.92$ & $\star$ & $33.0 ; 2.15$ & $28.5 ; 4.32$ \\
\hline
\end{tabular}

Table 2: Survey table of separation locations and correlation lengths near trailing edge at $x / C=83 \%$ for RIS $\varnothing-\mathrm{C} 2-18$ airfoil. Table values indicate: $x_{\mathrm{sep}} / C[\%]$; Measured $L_{x}[\mathrm{~cm}]$; Modelled $L_{x}[\mathrm{~cm}]$ (Modelled $L_{x}$ values according to Eq. (5) only for clean airfoil; *: no separation detected; n/a: experimental data not available) 

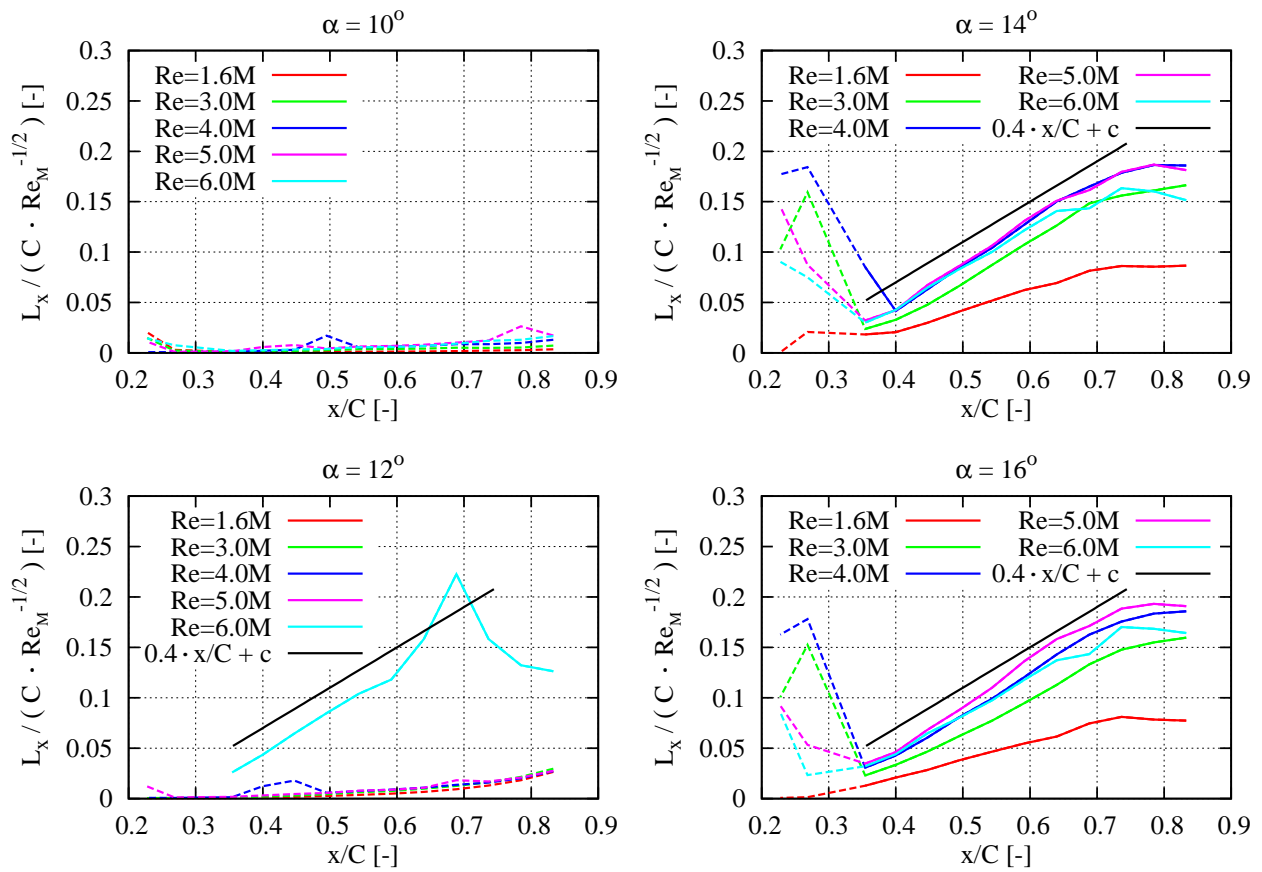

(a) Clean airfoil
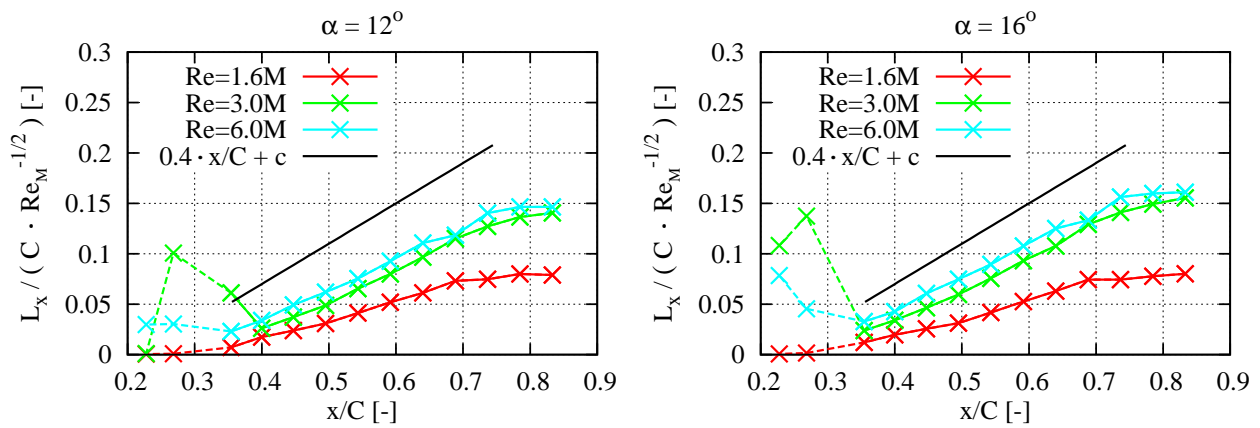

(b) ZZ tape
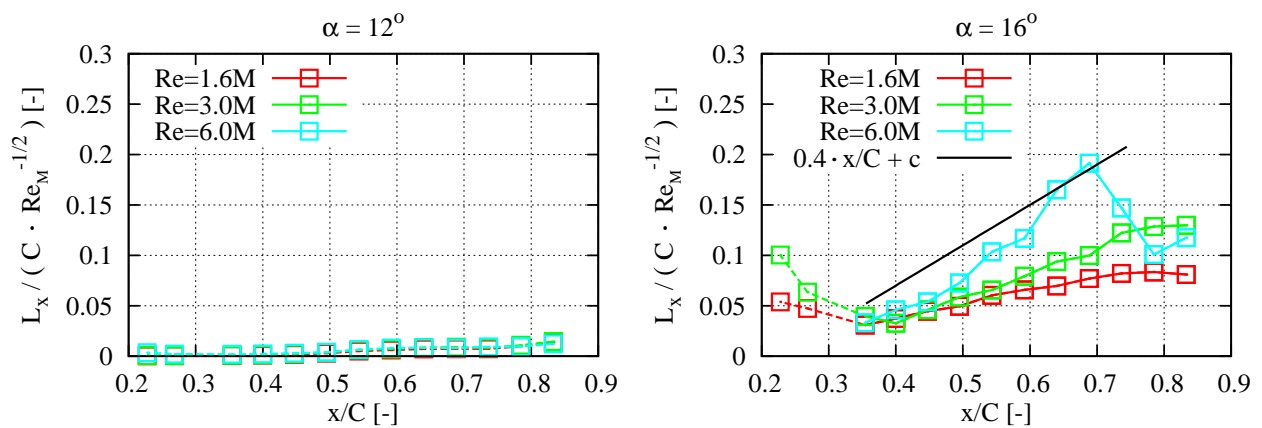

(c) Turbulence grid

Figure 11: RISØ-C2-18 airfoil - Correlation length along chord (Dashed lines: attached flow 

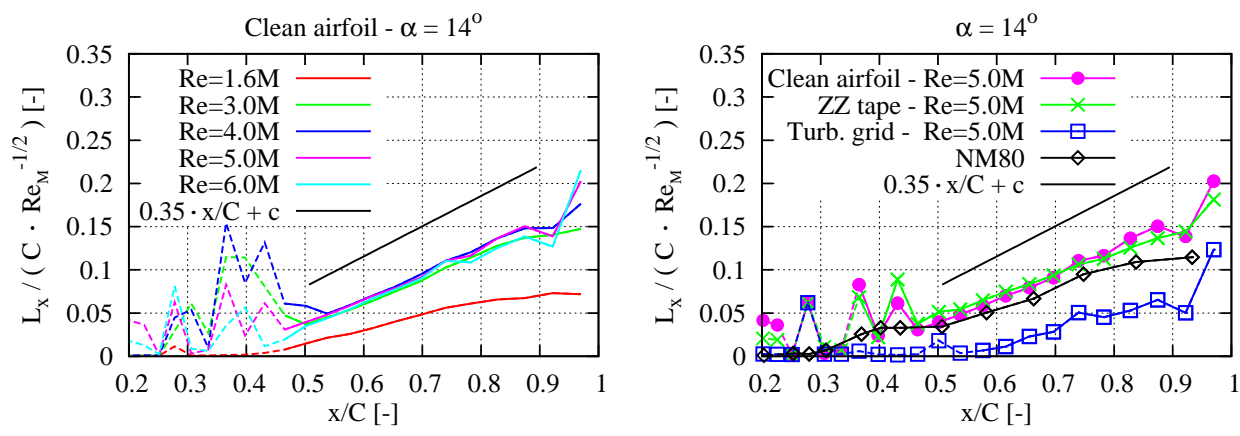

Figure 12: NACA-63-418 airfoil - Correlation length along chord (Dashed lines: attached flow

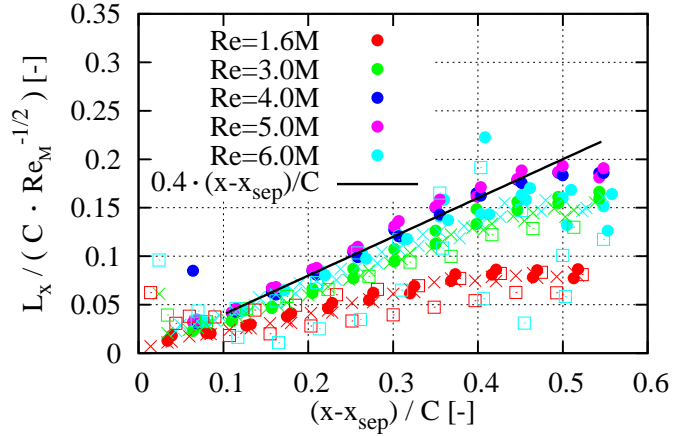

(a) RISØ-C2-18

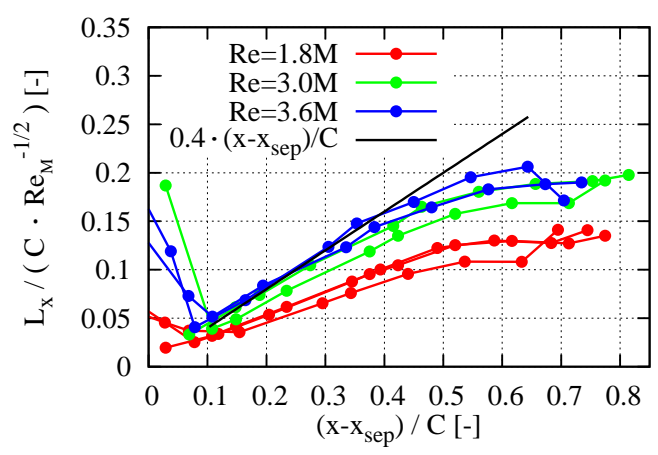

(c) NACA-0015

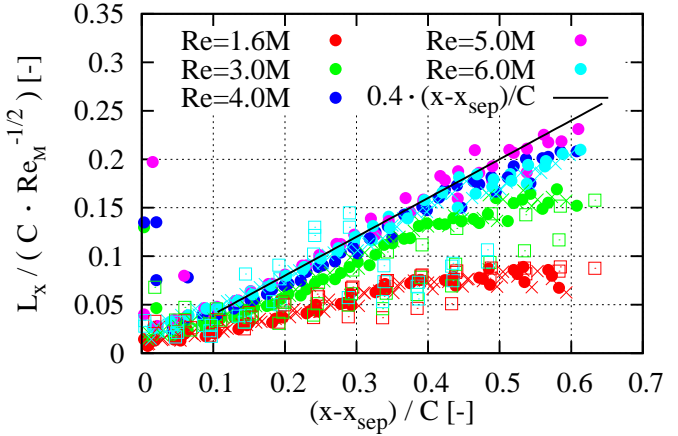

(b) RISØ-B1-18

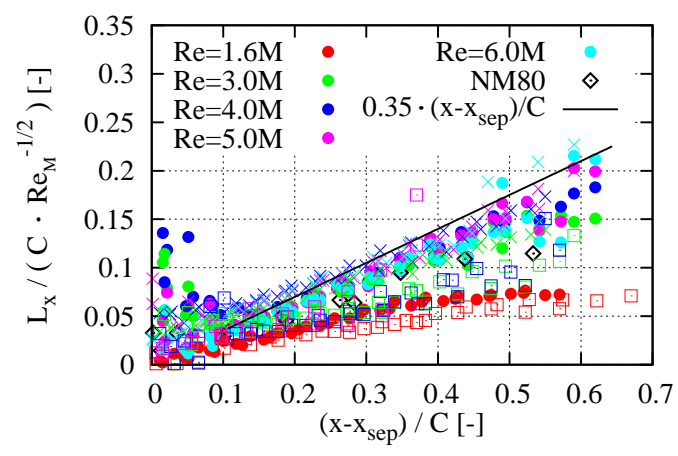

(d) NACA-63-418

Figure 13: Correlation length along chord - Scatter plot (Circles: clean airfoil; Crosses: ZZ tape; Squares: turbulence grid) 

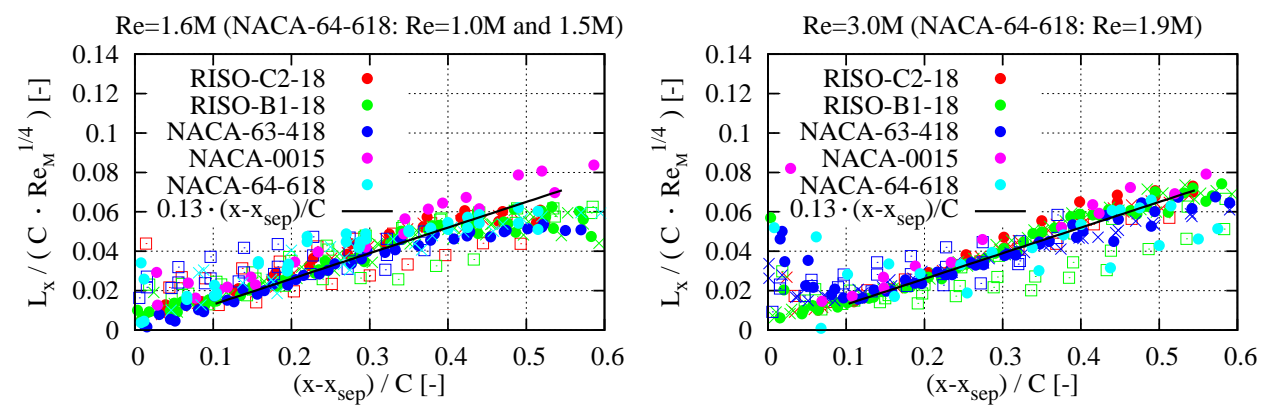

Figure 14: Correlation length along chord for $R e=1.6 \times 10^{6}$ and $3 \times 10^{6}$ - Scatter plot (Circles: clean airfoil; Crosses: ZZ tape; Squares: turbulence grid) 


\subsection{Coherence Function in Chord Direction and its Scaling}

The correlation characteristics in the chord direction are now investigated using the cross-spectra of the SP measured at various locations along the airfoil chord.

Let $\tilde{p}\left(\omega, x_{i}\right)$ denote the temporal Fourier transform of the SP time-series recorded at the chord position $x_{i}$, where $x_{i}$ refers to any microphone as displayed in Fig. 4 and $\omega$ is the circular frequency. The cross-spectrum between the time-series recorded at a pair of microphones located at the chord positions $x_{i}$ and $x_{j}$ reads:

$$
\Phi\left(\omega, x_{i}, x_{j}\right)=<\tilde{p}\left(\omega, x_{i}\right) \cdot \tilde{p}^{*}\left(\omega, x_{j}\right)>
$$

where $\tilde{p}^{*}$ is the complex conjugate of $\tilde{p}$. The coherence function between these microphones is then defined as:

$$
\gamma_{x_{i}}(\omega, \xi)=\frac{\left|\Phi\left(\omega, x_{i}, x_{j}\right)\right|}{\sqrt{\left|\Phi\left(\omega, x_{i}, x_{i}\right)\right| \cdot\left|\Phi\left(\omega, x_{j}, x_{j}\right)\right|}}
$$

where $\xi=x_{j}-x_{i}$. The function $\gamma_{x_{i}}$ is here understood as the coherence between a specific microphone numbered $i$ and any other microphone $j$ along the chord.

The measured coherence functions for the RIS $\varnothing-\mathrm{C} 2-18$ airfoil are plotted at three Reynolds numbers and three chord locations for $\alpha=16^{\circ}$ in Fig. 15. Similar figures could be obtained for different AoAs or airfoil sections, as long as the considered microphones are located in the stalled flow region. However, for the sake of brevity these are not reproduced here. The measured functions for the different airfoil sections, flow conditions and microphone locations, do collapse if the chordwise separation length $\xi$ is normalized using the local correlation length $L_{x}$ as defined in the previous section. In addition, the frequency is normalized as a Strouhal number based on the freestream velocity and the local correlation length and defined as:

$$
S t_{L}=f \cdot L_{x} / U_{\infty}
$$

where $f=\omega /(2 \pi)$ is the natural frequency. It is important to note that this Strouhal number is implicitly dependent on the chord location through the correlation length $L_{x}$ (see previous section) and that the measured $L_{x}$ are used for the normalizations. In Fig 15, a flow pattern characterized by a coherence hump centered can be observed for 
all airfoil chord locations and all Reynolds numbers around $S t_{L} \approx 0.1$ and it extends spatially to $\xi / L_{x} \approx \pm 6$. A detailed study showed that the center Strouhal number for this coherence hump is roughly independent of the airfoil profile, chord location, AoA and Reynolds number.

The functional form proposed in order to model the coherence function $\gamma_{x_{i}}$ reads:

$$
\gamma_{1}(\omega, \xi)=\mathrm{e}^{-\left[a_{x}\left(\xi / L_{x}\right)^{2}\left(S t_{L}-S t_{x}\right)^{2}+b_{x}\left(\xi / L_{x}\right)^{2}\right]}
$$

It is inspired by the spanwise coherence model proposed by Moreau et al [11] (see Sec-

and the resulting optimized model constants are:

$$
a_{x}^{<}=40.0, \quad a_{x}^{>}=5.22, \quad b_{x}=0.0586, \quad \text { and } \quad S t_{x}=0.0725
$$

The model coherence function $\gamma_{1}$ is displayed for the same three chord locations at the bottom of Fig. 15 and compares well with the plots obtained from the measurement data of the RIS $\varnothing-\mathrm{C} 2-18$ airfoil section.

\subsection{Coherence Function in Span Direction and its Scaling}

The correlation characteristics in the span direction are investigated using the crossspectra of the SP measured at various span locations but at the same chord position. 
Let $\tilde{p}(\omega, y)$ denote the temporal Fourier transform of the SP time-series recorded at different span positions $y$. There are 7 SP microphones distributed spanwise at positions $y_{0}+\eta_{i}(i=0, \ldots, 6)$. The cross-spectra between the time-series recorded at the reference microphone defined by $\eta_{0}=0$ and at any other span location read:

$$
\Phi\left(\omega, y_{0}, y_{0}+\eta\right)=<\tilde{p}\left(\omega, y_{0}\right) \cdot \tilde{p}^{*}\left(\omega, y_{0}+\eta\right)>
$$

and the spanwise coherence function between these pairs of microphones is defined as:

$$
\gamma_{y_{0}}(\omega, \eta)=\frac{\left|\Phi\left(\omega, y_{0}, y_{0}+\eta\right)\right|}{\sqrt{\left|\Phi\left(\omega, y_{0}, y_{0}\right)\right| \cdot\left|\Phi\left(\omega, y_{0}+\eta, y_{0}+\eta\right)\right|}}
$$

where the function $\gamma_{y_{0}}$ is understood as the coherence function relatively to the reference microphone located at $y_{0}$.

The coherence functions calculated from measurements on the NACA-64-618 airfoil are plotted in Fig. 16 for microphones located at $x / C=0.975$ when the flow is stalled. The frequency axis is non-dimensionalized using the Strouhal number defined in Eq. (6) and the spanwise separation length $\eta$ is non-dimensionalized using the local correlation length $L_{x}$. It can be seen that using the above non-dimensionalizations, all plotted coherence functions are very similar to each other independently of Reynolds number, AoA or even the use of a ZZ tape. Note that the spatial extent along the span displayed in the figure covers the entire microphones distribution spanwise. It is clear that it is too small to capture the whole range where the coherence function has non-negligible values. The same remark holds for the lowest frequencies, yet the frequency resolution allows to capture the function peaks all located around $S t_{L} \approx 0.1$. The plots have also been purposedly truncated for Strouhal numbers higher than $S t_{L}>1$ where the functions reach negligible values. The remaining of the coherence function at larger spanwise separation lengths and lower frequencies is still unknown. This is a potential source of errors when deriving a coherence model as the function tuning (see below) will not include these unknown values. Nevertheless, a functional form similar to a previous work from the literature [11], which has been itself validated against experimental data, will be used herein as a coherence model. If the measured part of the coherence function is well predicted by the model, then it can be surmised that a sensible model approximation of the whole function range has been obtained. 
Similarly to the model proposed by Moreau et al [11], the following model function optimized in order to fit the measured coherence functions displayed in Fig. 16. The model function is also plotted in this figure and exhibits strong similarity with the measured functions. The main difference between the present functional form and that chosen by Moreau et al [11] lies in the fact that in our case the Strouhal number at which the coherence reaches its peak is defined by the model parameter $S t_{y}$. Instead, Moreau et al [11] use a multiplicative factor in order to enforce the convergence of the coherence to zero in the limit of lower frequencies. This peak Strouhal number is directly related to the physics of the flow which we believe to be universal. It seems therefore natural to explicitly introduce this quantity as a model parameter. A second important difference is that, in the present model, the decay of the coherence function as a function of the span coordinate depends on the correlation length in the chord direction $L_{x}$. It is thereby assumed that the sizes of the turbulent vortices in both directions are related.

The model function $\gamma_{2}$ defined in Eq. (8) is compared with the measurement and model data of the spanwise correlation published by Moreau et al [11] for a NACA0012 airfoil in Fig. 17. The inputs for the present model replicate their experimental conditions. The separation location is approximated at $x_{\mathrm{sep}} / C=5 \%$ as it is not specified in [11]. Eq. (4) is used to evaluate the correlation length $L_{x}$ in Eq. (8). The present model reproduces fairly well the broadband part of the measurement data and model in [11]. The two distinct peaks of the narrow-band part are not captured by the present model, but these were neither observed in the present measurements. This may suggest that they are specific to lower Reynolds number flows as experienced in [11].

Finally, as it will appear later in this article when deriving a stall noise model, the wavenumber Fourier transform of the $\gamma_{2}$ function is required instead of a function of 
the spanwise separation length. It can be shown that:

$$
\Gamma_{2}\left(\omega, k_{2}\right)=\frac{1}{2 \pi} \int_{-\infty}^{+\infty} \gamma_{2}(\omega, \eta) \mathrm{e}^{-\mathrm{i} k_{2} \eta} \mathrm{d} \eta=\frac{c_{y}(\omega)}{\pi\left(c_{y}(\omega)^{2}+\omega^{2}\right)}
$$

where $c_{y}(\omega)=\left(a_{y}\left(S t_{L}-S t_{y}\right)^{2}+b_{y}\right) / L_{x}$. Once again, as a function of the quantities $L_{x}$ 545 and $S t_{L}$, the above function implicitly depends on the chord location. 

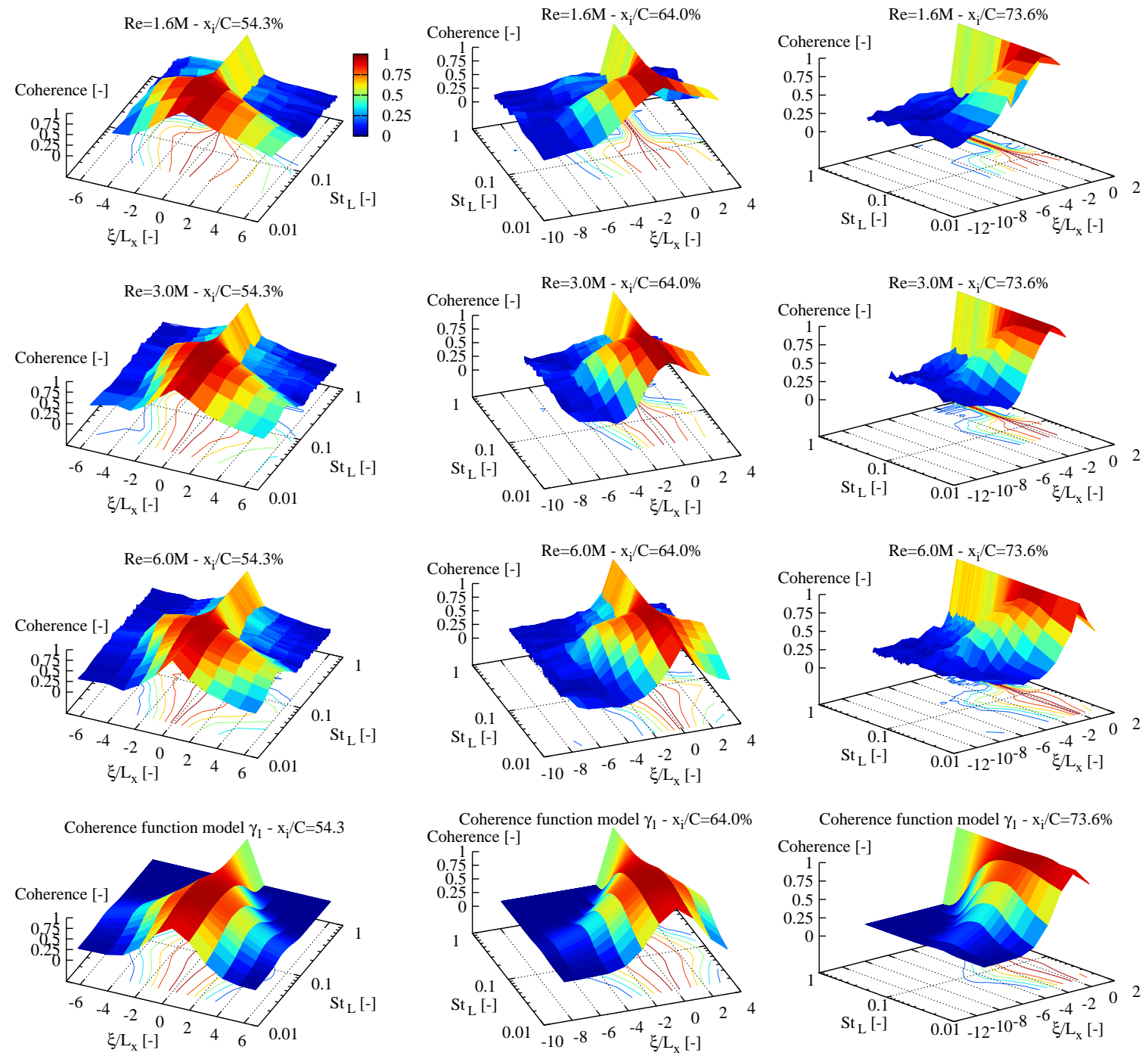

Figure 15: RISØ-C2-18 - Coherence function along chord (Row \#1: $R e=1.6 \times 10^{6}$; Row \#2: $R e=3 \times 10^{6}$; Row \#3: Re $=6 \times 10^{6}$; Row \#4: Model as in Eq. (7); Column \#1: $x / C=54 \%$; Column \#2: $x / C=64 \%$; Column $\# 3: x / C=74 \%$ ) 

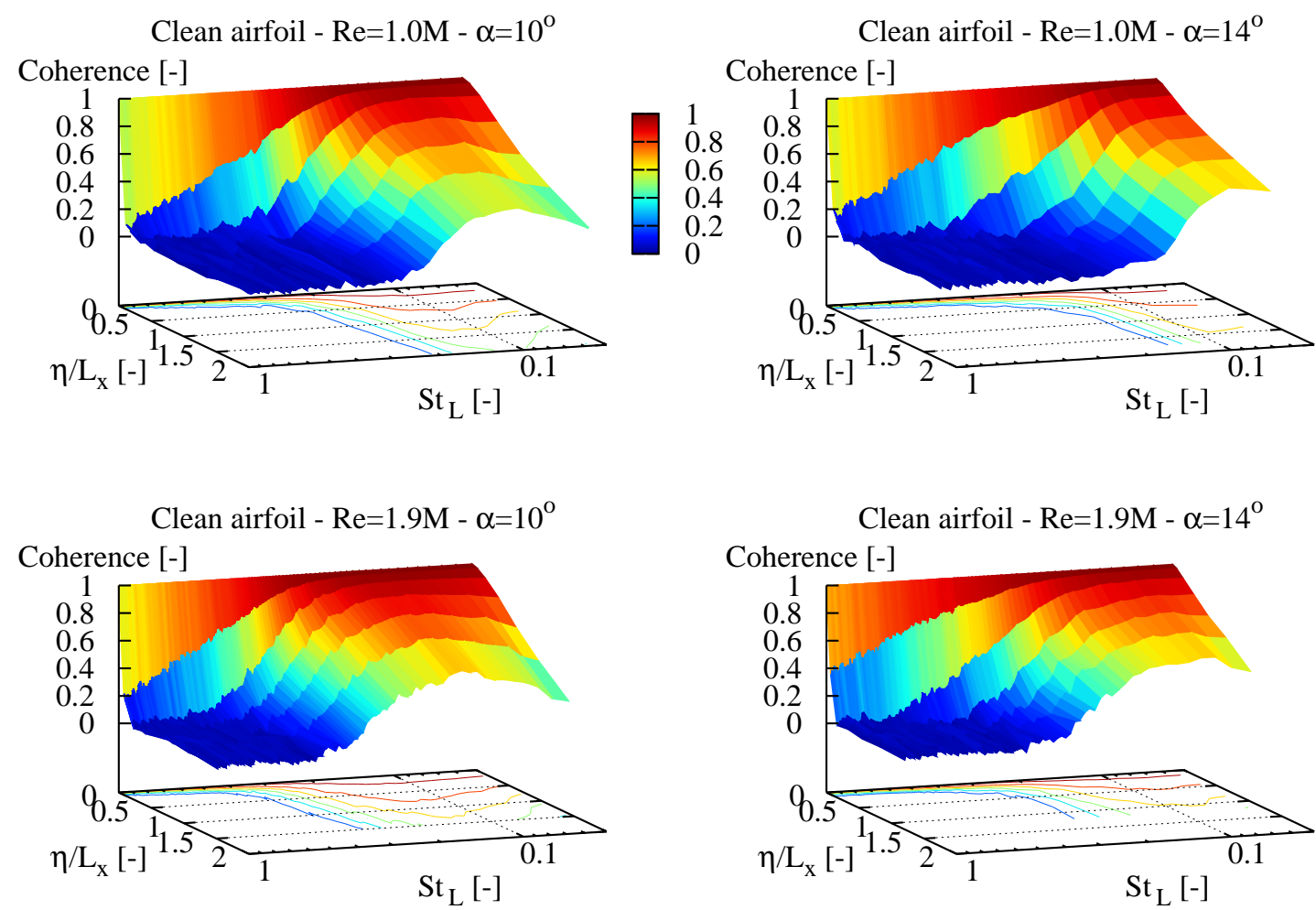

Clean airfoil $-\operatorname{Re}=1.9 \mathrm{M}-\alpha=14^{\circ}$

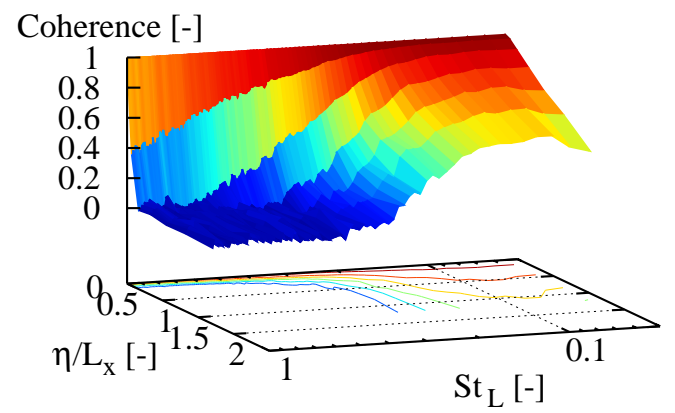

Coherence function model $\gamma_{2}$

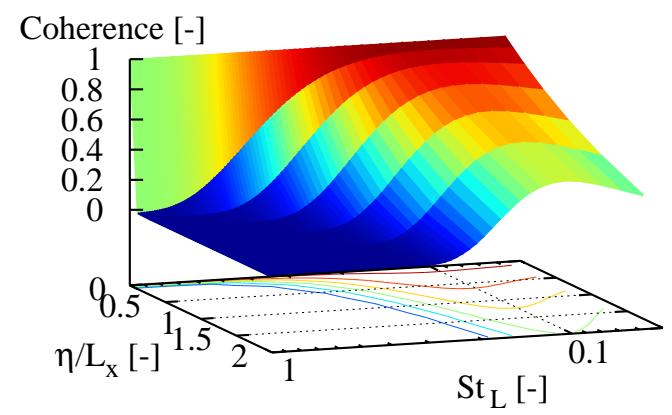

$$
\mathrm{ZZ} \text { tape }-\mathrm{Re}=1.5 \mathrm{M}-\alpha=14^{\mathrm{o}}
$$

Coherence [-]

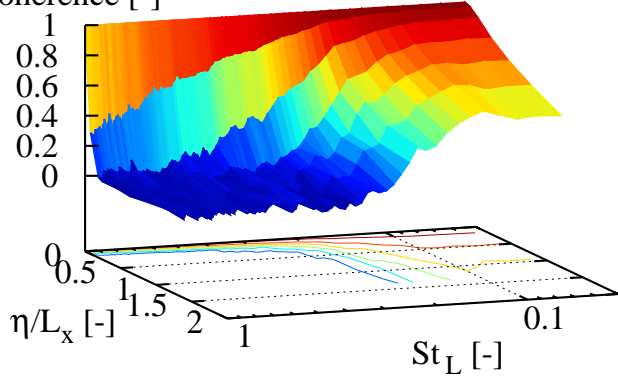

Figure 16: NACA-64-618 - Coherence function along span 


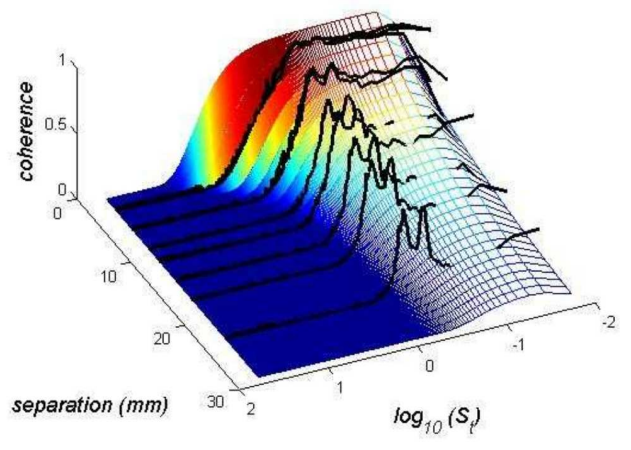

(a) Spanwise coherence and broadband model (Figure from Moreau et al [11])

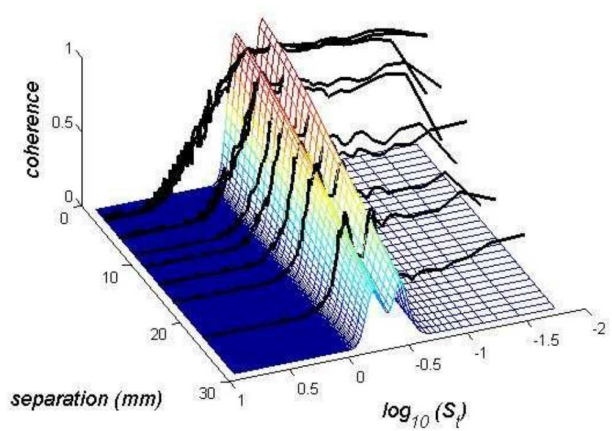

(b) Spanwise coherence and narrow-band model (Figure from Moreau et al [11])

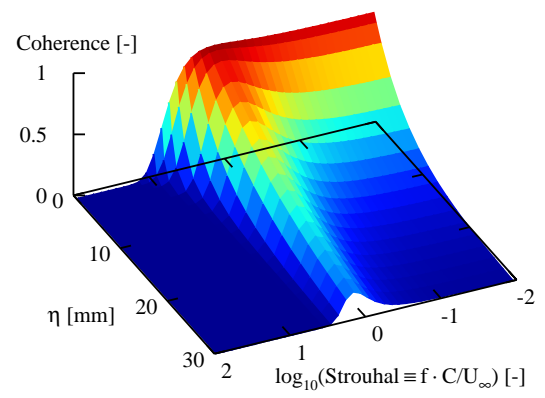

(c) Coherence function $\gamma_{2}$ as in Eq. (8) with input data from [11]

Figure 17: Coherence function along span for NACA-0012 airfoil 


\section{Surface Pressure Spectrum in Detached Stalled Flow Region}

The main building block of the stall noise model is now investigated, namely the SP spectra in the detached flow region of the stalled airfoils.

\subsection{Spectral Characteristics of Stall} microphone is upstream of the separated flow region at both Reynolds numbers. The SP spectra are hardly modified at $R e=6 \times 10^{6}$, however at $R e=1.6 \times 10^{6}$ a significant increase of the spectral energy centered around $f=300 \mathrm{~Hz}$ can be observed, as well 
as an increase in the low frequency range for $f<80 \mathrm{~Hz}$. This reflects the fact that flow separation is now occuring very close to this microphone for this latter Reynolds number as observed in Fig. 18(a). For larger AoAs $\alpha=12^{\circ}$ and $14^{\circ}$, broad spectral humps located at lower frequencies $30<f<300 \mathrm{~Hz}$ clearly emerge as both microphones are now located inside the separated flow region.

In Fig. 18, the background noise spectra measured by the microphone in the equalizing pressure slit are plotted together with spectra measured by the downstream microphone at $x / C=92 \%$. Those measured at $x / C=1.6 \%$ on the airfoil pressure side, near the leading edge stagnation point, are plotted together with the upstream microphone spectra at $x / C=61 \%$. It can be seen that the slit and leading edge microphones spectra are often well below (approximately one decade) the SP measurements at the two other microphones up to $1 \mathrm{kHz}$. Above this frequency, background noise clearly contaminates the measured spectra as large spectral peaks originating from the wind tunnel fan, among other possible perturbating sources, emerge. This is mainly the case for the stalled cases as the spectral energy at these frequencies is considerably reduced in stalled conditions compared to the attached flow, in particular for the upstream microphone. The background noise may also contaminate the results at very low frequencies $f<20 \mathrm{~Hz}$ as shown by the leading edge microphone measurements.

In addition, the above SP spectra at $R e=1.6 \times 10^{6}$ are compared in Fig. 18(c) to those measured by Christophe et al [12] for the CD airfoil at $R e=1.6 \times 10^{5}$. For a meaningful comparison, the frequency is non-dimensionalized using the inflow velocities and airfoil chords, and the SP spectra are displayed as sound pressure levels scaled with $U_{\infty}^{3}$ which appear to be the proper scaling for these Reynolds number regimes as shown in the next section. It can be seen that for $\alpha=6^{\circ}$ and $8^{\circ}$, the NACA-63-418 and the CD airfoils, respectively, exhibit the same SP plateau in the low frequency range. The spectral roll-offs in the high frequency range scale differently, although not the transition frequency between the two ranges and the slopes are similar. For higher AoAs, both airfoils exhibit the previously observed spectral hump at low frequencies characteristic of stall. It can be concluded that these characteristics seem universal, although the AoA at which they are observed may vary from one airfoil to the other. 

length in Section 5.1, it is found that the spectra measured at $R e=1.6 \times 10^{6}$, and somehow for $R e=3 \times 10^{6}$ in some cases, clearly depart from the higher Reynolds

For the sake of brevity, in this section data are gathered in a few figures by plotting all or most of these data as scattered dots without discriminating between Reynolds number, AoA or microphone chord location, so that scaling trends can be observed if such exist. In some cases, a single scatter plot even includes data measured not only on a clean airfoil, but also with ZZ tape or turbulence grid. Yet, the main requirement for the plotted data is that the measuring microphone is located within the separated region of the stalled flow.

Firstly, the data scaling consists in non-dimensionalizing the frequency-axis using the Strouhal number $S t_{L}$ as introduced in Section 5.2. The measured $L_{x}$ are used for all scalings in this section. Secondly, the measured SP power spectral densities are normalized in accordance with the above frequency scaling and further non-dimensionalized using the freestream dynamic pressure. Finally, the normalized spectra are divided by a scaling factor $R e_{M}^{-5 / 2}$ where the reduced Reynolds number $R e_{M}$ has been defined in Section 5.1. In summary, if $S_{p p}(f)$ is the measured SP power spectral density, then the normalized and scaled power spectral densities read:

$$
S_{p p}^{(+)}\left(S t_{L}\right)=\frac{S_{p p}(f)}{q_{\infty}^{2} \cdot L_{x} / U_{\infty}} \cdot \frac{1}{R e_{M}^{-5 / 2}}
$$

where $q_{\infty}=0.5 \rho U_{\infty}^{2}$ is the dynamic pressure. Note that all plotted spectra in this article are one-sided, i.e. they have been multiplied by a factor 2 to account for the energy of their symmetric part.

Spectra are plotted using wind tunnel data of four airfoils, namely the RIS $\varnothing-C 2-18$, RISØ-B1-18, NACA-0015 and NACA-63-418, as well as data from the NM80 turbine measurements together with the fourth airfoil, in Figs. 19(a-b-c-d), respectively. It can be observed that there exists a general trend of the spectra measured at high Reynolds numbers from $R e=3 \times 10^{6}$ to $6 \times 10^{6}$ to coalesce around a common curve below approximately $S t_{L} \lesssim 0.2$. Some scatter also exists. It may be attributed to experimental noise and effects of the chaotic behavior of stall vortices. As noticed for the correlation

\subsection{Scaling Properties}


number spectra (see further investigation below). In view of the preliminary study characterizing the spectral shape of SP measurements from microphones located in the stalled flow region, the above scaling property is obviously characteristic of the stall phenomenon. Above $S t_{L} \gtrsim 0.2$ the scatter grows considerably and the spectra coalesce no longer. This latter issue is investigated later on.

The effects of the ZZ tape and of the turbulence grid can be evaluated for the RIS $\varnothing$ C2-18, RIS $\varnothing-B 1-18$ and NACA-63-418/NM80 airfoils in Figs. 20(a-b-c), respectively. The ZZ tape has a tendency to improve the merging of the results obtained at $R e=3 \times 10^{6}$ using the above scaling. The turbulence grid somehow flattens the spectral hump and it appears to be in good agreement with the measurements on the NM80 wind turbine blade which is naturally exposed to turbulence from the atmospheric inflow.

As noticed above, the spectra measured at lower Reynolds numbers do not follow the proposed scaling. Fig. 21 gathers spectra measured on the four previous airfoils at $R e=1.6 \times 10^{6}$ and $3 \times 10^{6}$, together with the measurements performed on the NACA-64618 at $R e=1.0 \times 10^{6}, 1.5 \times 10^{6}$ and $1.9 \times 10^{6}$. In these cases, it is found that a different scaling must be used in order for the spectra to coalesce. It reads:

$$
S_{p p}^{(-)}\left(S t_{L}\right)=\frac{S_{p p}(f)}{q_{\infty}^{2} \cdot L_{x} / U_{\infty}}
$$

This finding strengthens the hypothesis of a change of regime from lower to higher Reynolds numbers as discussed in Section 5.1. It is interesting to note that Moreau et al [11] reported SP spectra scaling as $U_{\infty}^{3}$ for their measurements performed at Reynolds number lower than $1 \times 10^{6}$, which is consistent with the present scaling for low Reynolds numbers. However, it appears in Fig. 21 that the measured SP for the NACA-64-618 obey this scaling but the spectral peak energy levels are nearly a decade lower and these peaks are much flatter than for the other airfoils. The reason for this discrepancy remains unclear. The wind tunnel configuration with Kevlar walls may play a role. The flat spectral peaks may indicate that stall is not fully established in accordance with the observations made in Section 6.1. It could also be that the proposed scaling is not universal. Nevertheless, comparisons of the SP and stall noise models with independent measurements in Section 8 will show that this scaling seems valid in many cases. 
The case of the spectral scaling at higher frequencies (i.e. $S t_{L} \gtrsim 0.2$ ) is now investigated. The change of spectral slope around the intermediate Strouhal number $S t_{L} \approx 0.2$ is studied by introducing a new scaling and changing the color convention for plotting the spectra. The spectra are now scaled using the distance between the microphone location $x$ and the separation location $x_{\text {sep }}$, instead of the correlation length $L_{x}$ as done previously. The resulting Strouhal number is defined as:

$$
S t_{S}=f \cdot\left(x-x_{\mathrm{sep}}\right) / U_{\infty}
$$

and the scaling for the SP spectra reads:

$$
S_{p p}^{(S)}\left(S t_{S}\right)=\frac{S_{p p}(f)}{q_{\infty}^{2} \cdot\left(x-x_{\mathrm{sep}}\right) / U_{\infty}}
$$

Furthermore, the color convention is now using the microphone chord locations to distinguish the different data. The spectra are plotted in Figs. 22(a) and (b) for the RIS $\varnothing-C 2-18$ and NACA-0015 airfoils, respectively. A transition from the spectral hump observed earlier at low frequencies to a linear roll-off (in log-log scale) at high frequencies is clearly observed. The Strouhal number at which this transition occurs is nearly constant and denoted as $S t_{S}^{\mathrm{t}} \approx 2$. Note that this transitional Strouhal number does not depend on the Reynolds number since spectra measured at all Reynolds numbers were included in the figures.

The color convention in the above figures clearly shows that the energy level of the linear roll-off at high frequencies depends on the chord location. This suggests that a new scaling is required to coalesce these parts of the spectra. Indeed, a scaling based on a combination of the airfoil chord and the distance to separation proves to be adequate for merging the SP spectra at frequencies larger than the above transitional frequency. A new Strouhal number is then defined as:

$$
S t_{A}=f \cdot\left(C^{2} /\left(x-x_{\mathrm{sep}}\right)\right) / U_{\infty}
$$

and the scaling for the SP spectra reads:

$$
S_{p p}^{(A)}\left(S t_{A}\right)=\frac{S_{p p}(f)}{q_{\infty}^{2} \cdot\left(C^{2} /\left(x-x_{\mathrm{sep}}\right)\right) / U_{\infty}}
$$


The measured and scaled spectra are plotted on Figs. 23(a) and (b) for the two airfoils considered above. Once again, similar results could be reproduced with the other airfoils but are not shown here.

\subsection{Surface Pressure Spectral Modelling}

In this section, the results of the previous measurement analyses are gathered to develop a general model for the SP frequency spectrum along the airfoil chord within the recirculating stalled flow region.

In Section 5.1, it is shown that the correlation length along the airfoil chord can be calculated as a function of the distance to the separation point. A model valid at any Reynolds number is proposed in Eq. (5) as a linear interpolation between two different scaling formulae corresponding to low and high Reynolds number regimes.

In the previous section, it is shown that the SP spectra can be roughly collapsed using proper scalings that depend on the Reynolds number and the correlation length for medium frequencies. The following functional forms are proposed for the high Reynolds number spectra:

$$
S_{p p}^{+}\left(S t_{L}\right)=2.5 \cdot 10^{-5} /\left(5 \cdot 10^{-6}+S t_{L}^{5}\right)
$$

as displayed in Figs. 19 and 20, and for the low Reynolds number spectra:

$$
S_{p p}^{-}\left(S t_{L}\right)=5 \cdot 10^{-7} /\left(3 \cdot 10^{-6}+S t_{L}^{5}\right)
$$

as displayed in Fig. 21. A model valid at any Reynolds number is formulated once again as a linear interpolation between the low and high Reynolds number regimes as:

$$
S_{p p}^{<>}\left(S t_{L}\right)=c_{R}\left(R e_{M}\right) S_{p p}^{-}\left(S t_{L}\right)+\left(1-c_{R}\left(R e_{M}\right)\right) S_{p p}^{+}\left(S t_{L}\right) \cdot R e_{M}^{-5 / 2}
$$

where the blending function $c_{R}$ has been defined in Section 5.1.

For $S t_{L} \lesssim 0.02$, all previously displayed spectra exhibit a transition to a smaller rolloff spectral slope toward small frequencies. The following functional form is proposed as a model for these low frequencies:

$$
S_{p p}^{<}\left(S t_{L}\right)=c_{p}^{<} \cdot \sqrt{S t_{L}}
$$


and is displayed in Fig. 23.

In summary, the SP stall model reads:

$$
S_{p p}(f)= \begin{cases}S_{p p}^{<}\left(S t_{L}\right) \cdot \frac{q_{\infty}^{2} L_{x}}{U_{\infty}} & \text { for } S t_{L}<0.02 \\ S_{p p}^{<>}\left(S t_{L}\right) \cdot \frac{q_{\infty}^{2} L_{x}}{U_{\infty}} & \text { for } 0.02<S t_{L} \text { and } S t_{S}<S t_{S}^{\mathrm{t}}, \\ S_{p p}^{>}\left(S t_{A}\right) \cdot \frac{q_{\infty}^{2} C^{2}}{U_{\infty}\left(x-x_{\mathrm{sep}}\right)} & \text { for } S t_{S}^{\mathrm{t}}<S t_{S} .\end{cases}
$$

Note that the transition between the medium and high frequency ranges is smoothed out in the numerical implementation of the model by linearly interpolating the two model functions over frequencies from $S t_{S}=S t_{S}^{\mathrm{t}} / \sqrt{2}$ to $S t_{S}=S t_{S}^{\mathrm{t}} \cdot \sqrt{2}$. Indeed, the two dimensionalized spectra are most likely to mismatch at $S t_{S}=S t_{S}^{\mathrm{t}}$. 

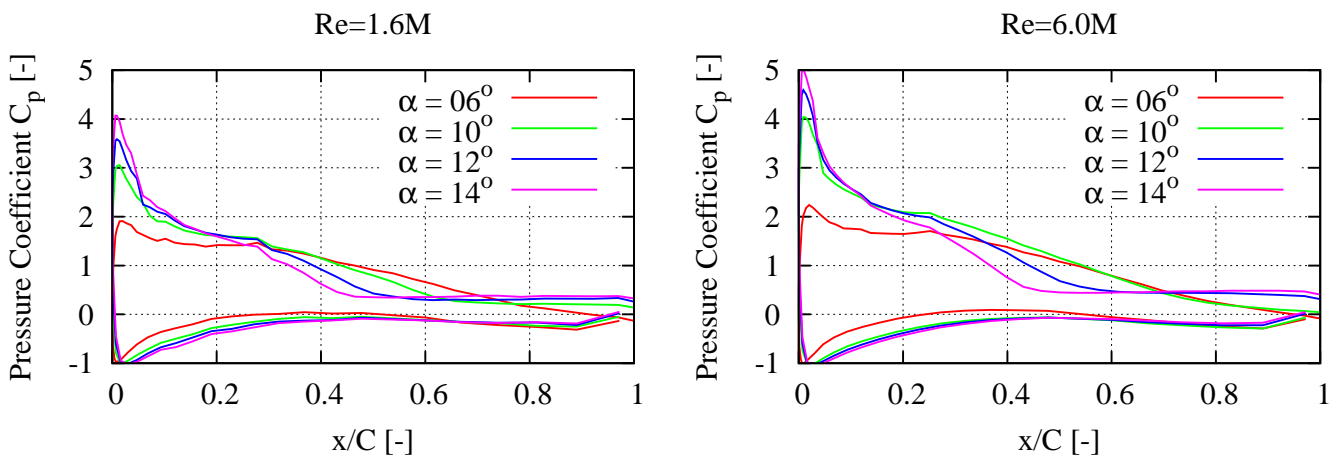

(a) Pressure coefficient distributions - NACA-63-418
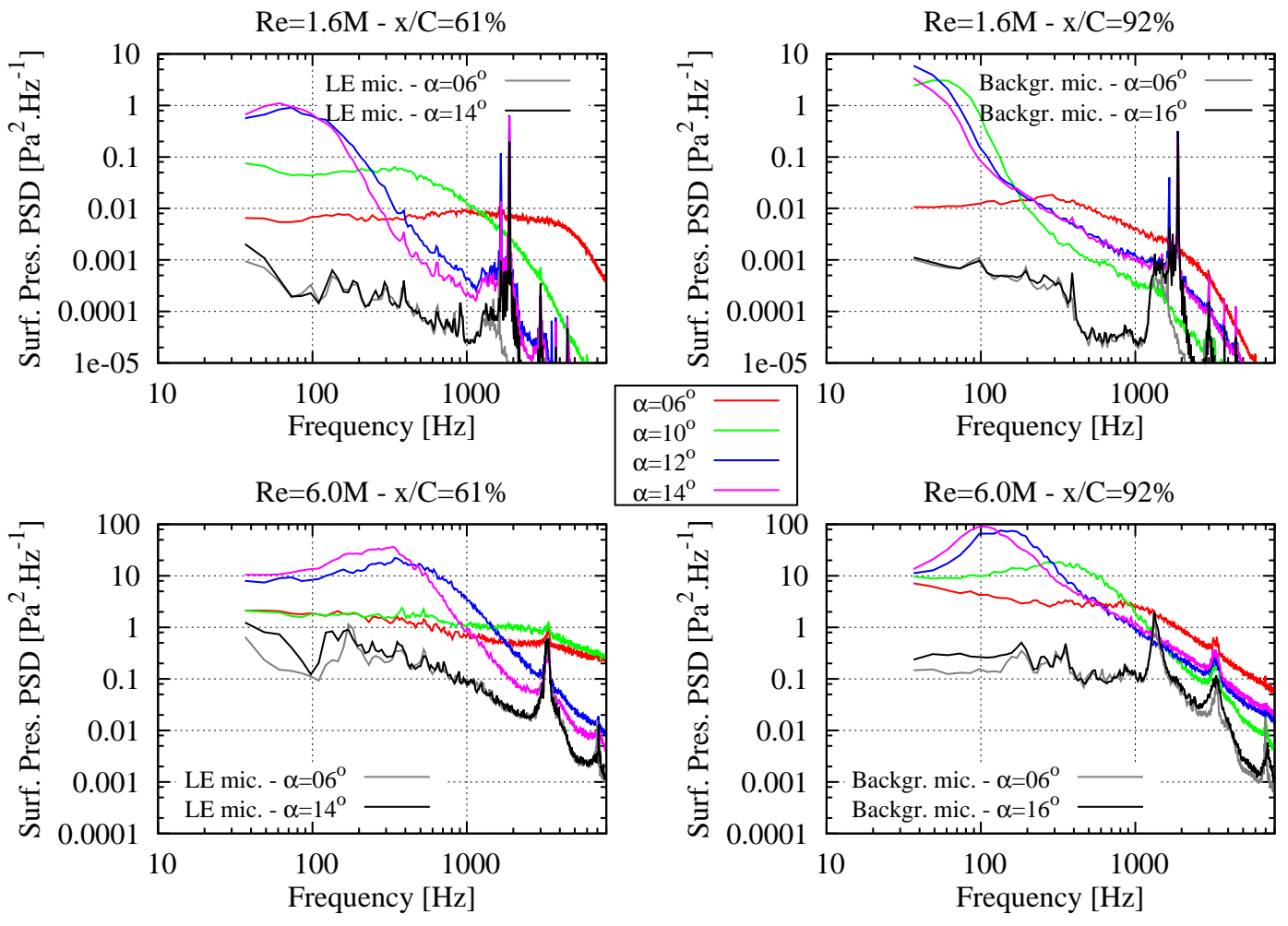

(b) SP spectra - NACA-63-418

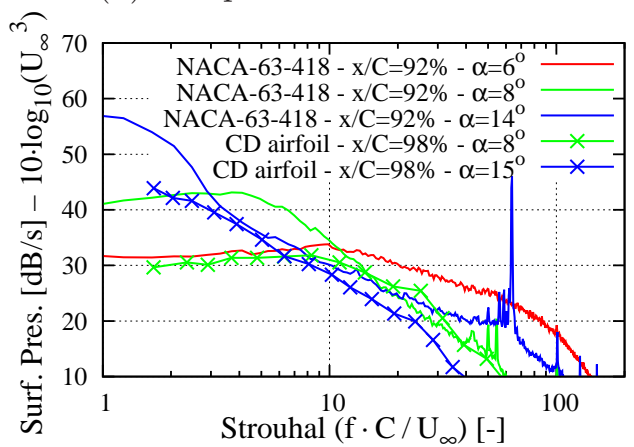

(c) NACA-63-418 and CD airfoil [12]

Figure 18: Characteristics of stall 


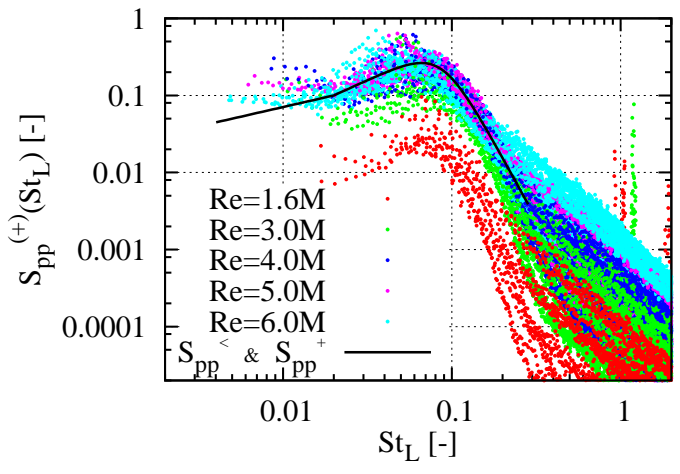

(a) RISØ-C2-18

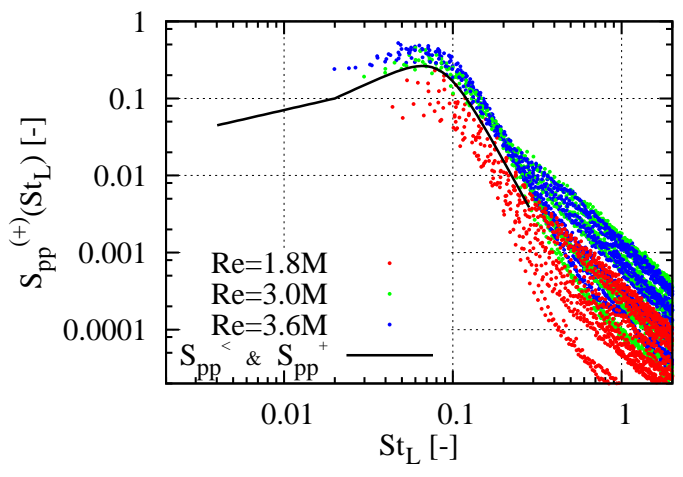

(c) NACA-0015

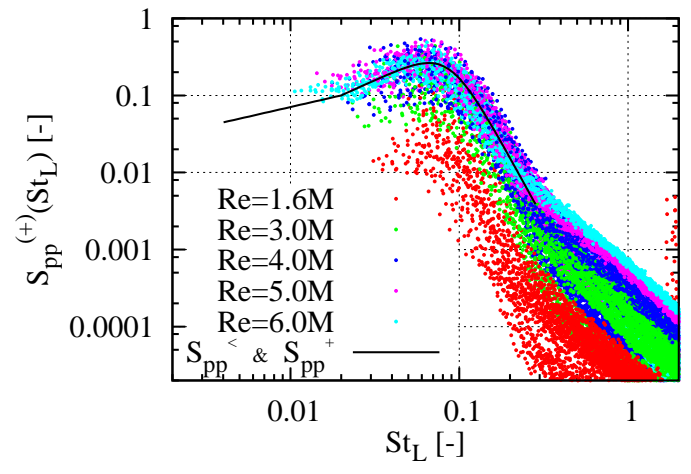

(b) RIS $\varnothing-B 1-18$

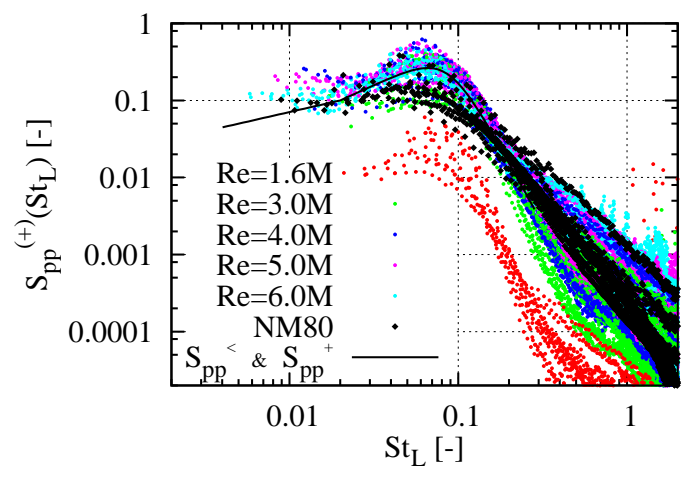

(d) NACA-63-418/NM80

Figure 19: Scaled SP spectra - Clean airfoils (Symbols: Measurements; Line: Prediction model $S_{p p}^{<}$from Eq. (12) and $S_{p p}^{+}$from Eq. (9) in Section 6.3) 

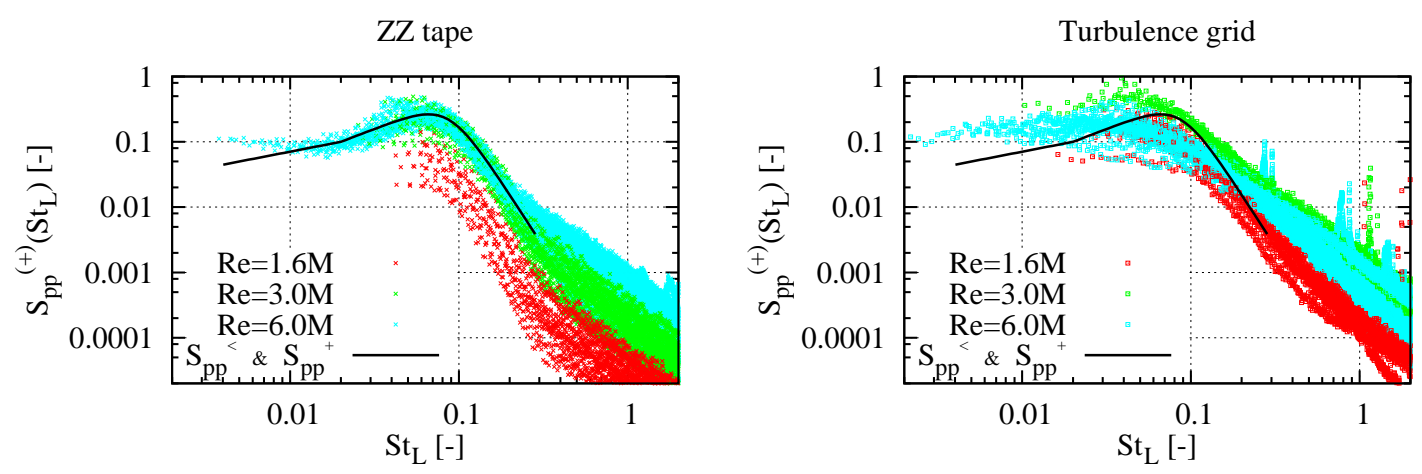

(a) RISØ-C2-18
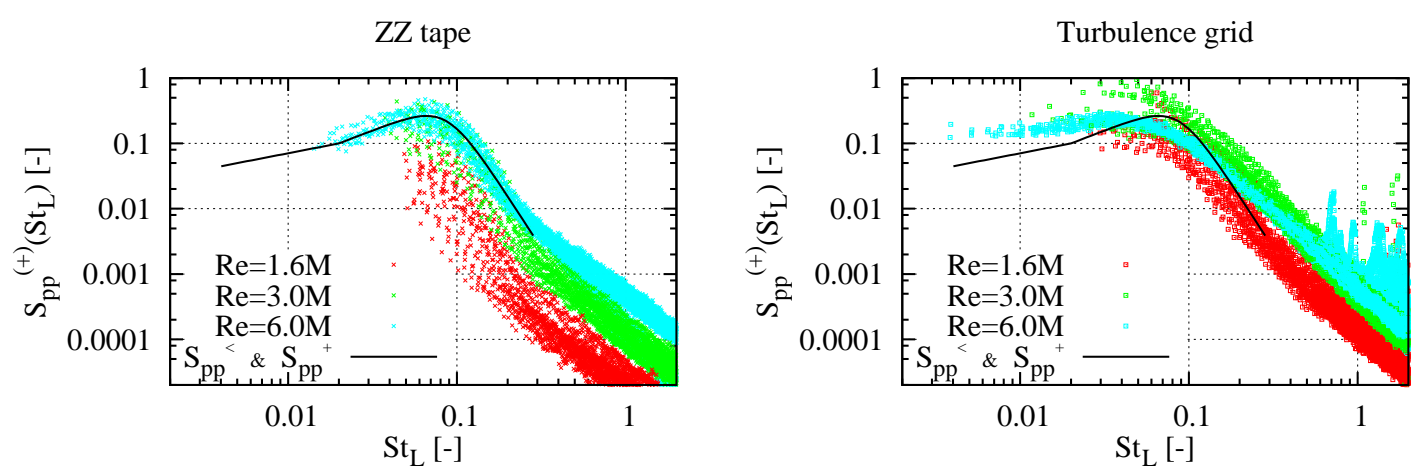

(b) RISØ-B1-18
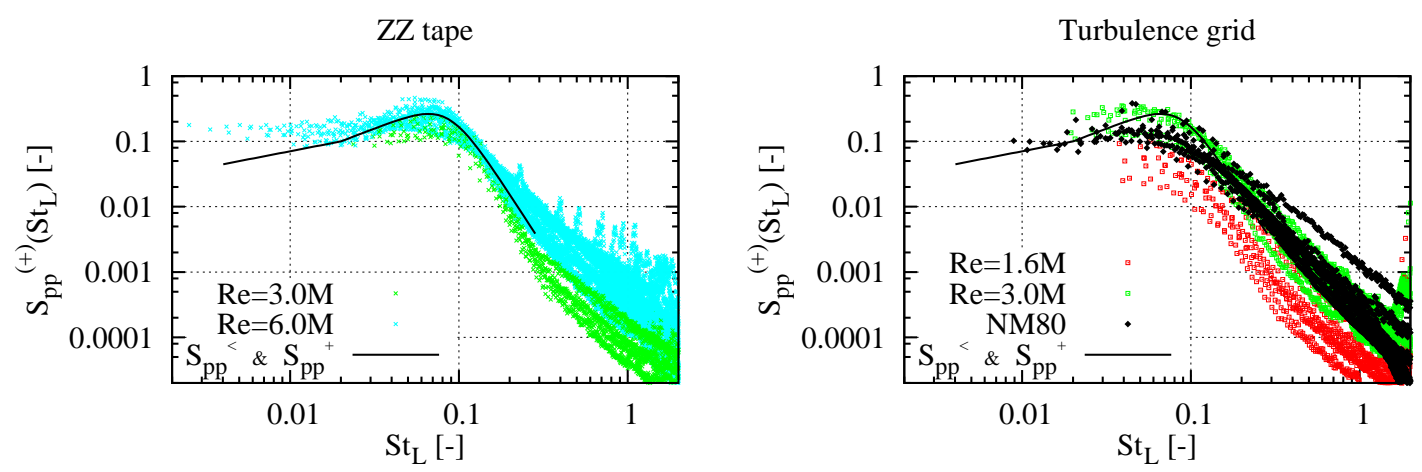

(c) NACA-63-418/NM80

Figure 20: Scaled SP spectra - ZZ tape and turbulence grid (Symbols: Measurements; Line: Prediction model $S_{p p}^{<}$from Eq. (12) and $S_{p p}^{+}$from Eq. (9) in Section 6.3) 


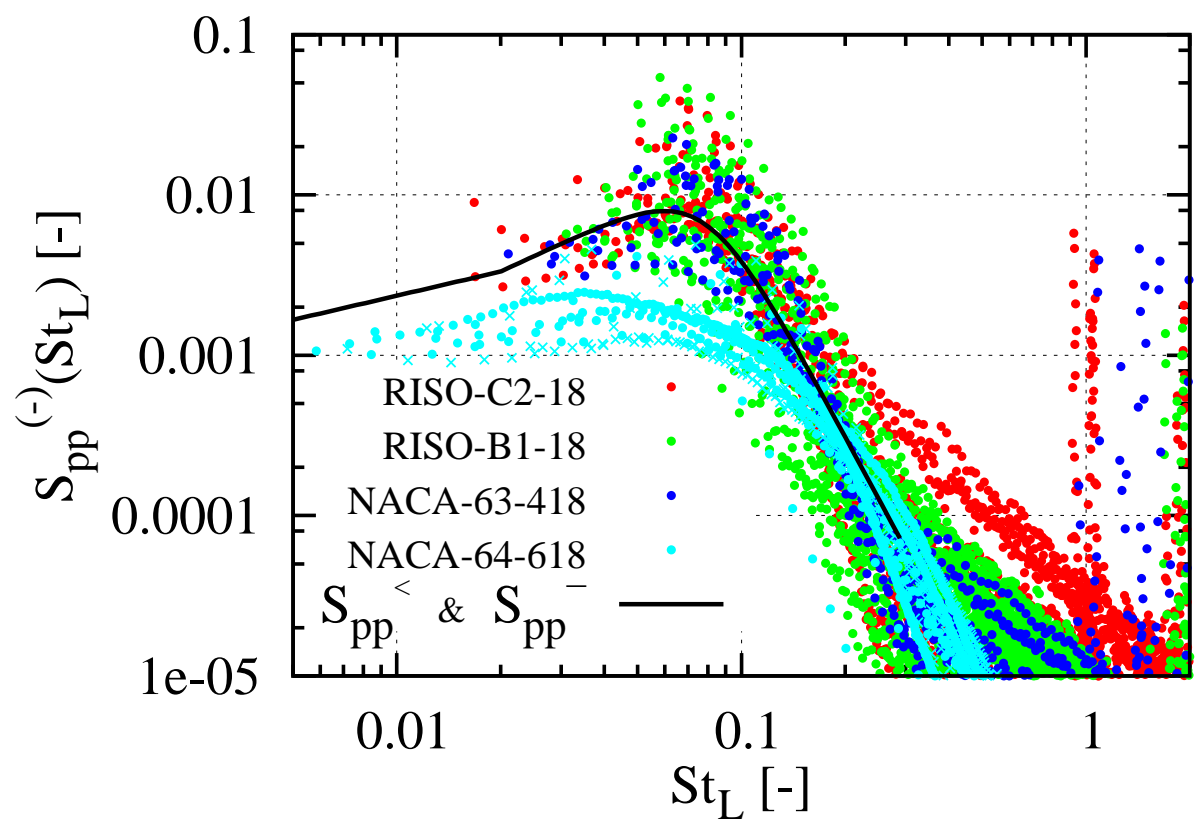

Figure 21: Scaled SP spectra at low Reynolds numbers (Symbols: Measurements; Line: Prediction model $S_{p p}^{<}$from Eq. (12) and $S_{p p}^{-}$from Eq. (10) in Section 6.3)

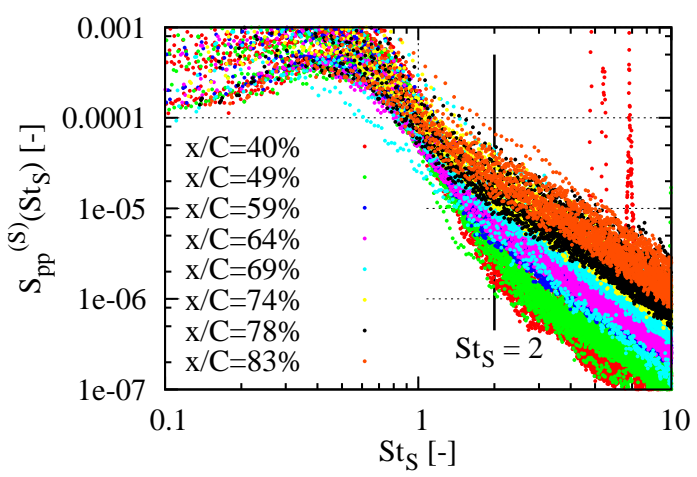

(a) RISØ-C2-18

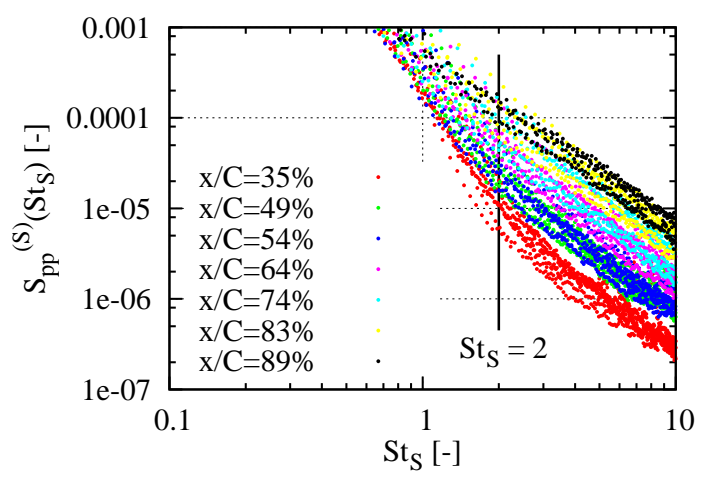

(b) NACA-0015

Figure 22: SP spectra scaled using distance from separation 


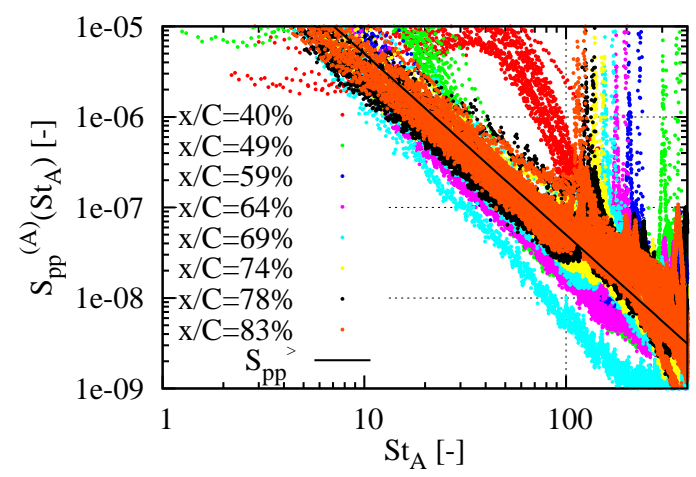

(a) RISØ-C2-18

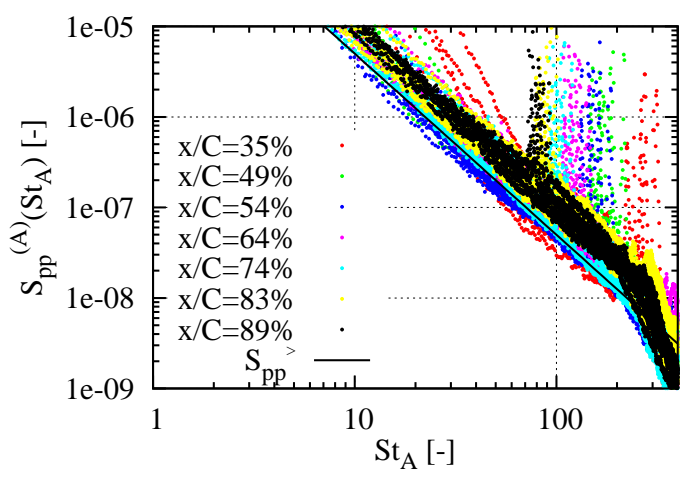

(b) NACA-0015

Figure 23: SP spectra scaled using $C^{2} /\left(x-x_{\text {sep }}\right)$ (Symbols: Measurements; Line: Prediction model $S_{p p}^{>}$from Eq. (13) in Section 6.3) 


\section{A Stall Noise Model}

Along the line of the stall noise model proposed by Moreau et al [11], our modelling approach is based on Curle's analogy [40] for an elementary dipole. Two model versions are developed: the first one is very similar to the derivation of Amiet's theory for trailing edge noise [41] and prescribes the SP spectrum at the trailing edge using the model developed in the previous section, the second one makes use of the fact that this model is valid in the whole separated stalled flow region along the airfoil chord at the cost of a more complex derivation.

\subsection{Model Basis}

The starting point for the model is the assumption that stall noise is generated by elementary dipole sources distributed across the airfoil surface within the stall flow region. The airfoil surface is approximated as a rectangular flat plate with chord and span lengths equal to $C$ and $L$, respectively. The origin of the reference system is set at the leading edge of the airfoil mid-span section, therefore its surface is defined as $S_{y}=[0, C] \times[-L / 2, L / 2]$. The acoustic pressure generated at a point $\boldsymbol{x}=\left\{x_{1}, x_{2}, x_{3}\right\}^{T}$ in the far-field ${ }^{1}$ by a dipole located at $\boldsymbol{y}=\left\{y_{1}, y_{2}, 0\right\}^{T}$ on the airfoil surface is given as:

$$
\tilde{p}_{a}(\omega, \boldsymbol{x})=\iint_{S_{y}} \frac{\mathrm{i} \omega R_{3}}{4 \pi c_{0} R_{s}^{2}} \tilde{p}(\omega, \boldsymbol{y}) \mathrm{e}^{-\frac{\mathrm{i} \omega R_{t}}{c_{0}}} \mathrm{~d} S_{y}
$$

where $\mathrm{i}$ is the complex imaginary unit, $c_{0}$ is the speed of sound and $d S_{y}=\mathrm{d} y_{1} \mathrm{~d} y_{2}$. In the above formula, the vector $\boldsymbol{R}=\left\{R_{1}, R_{2}, R_{3}\right\}^{T}$ is defined as $\boldsymbol{R}=\boldsymbol{x}-\boldsymbol{y}$ and $R_{s}^{2}=R_{1}^{2}+\beta_{x}^{2}\left(R_{2}^{2}+R_{3}^{2}\right)$, where $\beta_{x}^{2}=1-M_{x}^{2}$ and $M_{x}=U_{x} / c_{0}$ is the inflow Mach number computed from the inflow velocity vector projected onto the airfoil chord axis. The vector $\boldsymbol{R}_{t}$ is defined as $\boldsymbol{R}_{t}=\left\{\left(R_{1}-M_{x} R_{s}\right) / \beta_{x}^{2}, R_{2}, R_{3}\right\}^{T}$ and its norm is $R_{t}=\left|\boldsymbol{R}_{t}\right|$. Finally, $\tilde{p}(\omega, \boldsymbol{y})$ is the Fourier transform of the SP fluctuations acting on the airfoil surface, which takes the role of the dipole intensity in this context.

Assuming that the far-field noise can be considered as a stochastic process, its power spectrum can be evaluated as:

$$
S_{a}(\omega, \boldsymbol{x}) \delta\left(\omega-\omega^{\prime}\right)=<\tilde{p}(\omega, \boldsymbol{x}) \cdot \tilde{p}^{*}\left(\omega^{\prime}, \boldsymbol{x}\right)>
$$

\footnotetext{
${ }^{1}$ The superscript ${ }^{T}$ denotes the transpose operation.
} 
Integrating over the circular frequency $\omega^{\prime}$ and introducing Eq. (14) yields the far-field noise power spectrum:

$$
S_{a}(\omega, \boldsymbol{x})=\iint_{S_{y}} \iint_{S_{y}^{\prime}}\left(\frac{\omega}{4 \pi c_{0}}\right)^{2} \frac{R_{3} R_{3}^{\prime}}{R_{s}^{2} R_{s}^{\prime 2}}<\tilde{p}(\omega, \boldsymbol{y}) \cdot \tilde{p}^{*}\left(\omega, \boldsymbol{y}^{\prime}\right)>\mathrm{e}^{-\frac{\mathrm{i} \omega\left(R_{t}-R_{t}^{\prime}\right)}{c_{0}}} \mathrm{~d} S_{y}^{\prime} \mathrm{d} S_{y}
$$

This formula can be simplified by assuming that the listener's location $\boldsymbol{x}$ is far away from the airfoil surface. Using Taylor's expansion of the geometric factors and retaining only first order terms, the following approximations are obtained:

$$
\frac{1}{R_{s}^{2}} \approx \frac{1}{S_{0}^{2}} \quad \text { and } \quad R_{3} \approx x_{3}
$$

where $S_{0}^{2}=x_{1}^{2}+\beta_{x}^{2}\left(x_{2}^{2}+x_{3}^{2}\right)$. Furthermore, we have:

$$
R_{t}-R_{t}^{\prime} \approx \frac{1}{\beta_{x}^{2} S_{0}}\left[\left(x_{1}-M_{x} S_{0}\right)\left(y_{1}^{\prime}-y_{1}\right)+\beta_{x}^{2} x_{2}\left(y_{2}^{\prime}-y_{2}\right)\right]
$$

Introducing the above approximations in the far-field noise spectrum yields:

$$
\begin{array}{r}
S_{a}(\omega, \boldsymbol{x})=\left(\frac{\omega x_{3}}{4 \pi c_{0} S_{0}^{2}}\right)^{2} \iint_{S_{y}} \iint_{S_{y}^{\prime}}<\tilde{p}(\omega, \boldsymbol{y}) \cdot \tilde{p}^{*}\left(\omega, \boldsymbol{y}^{\prime}\right)>\cdots \\
\times \mathrm{e}^{-\mathrm{i} K_{1}\left(y_{1}^{\prime}-y_{1}\right)} \mathrm{e}^{-\mathrm{i} K_{2}\left(y_{2}^{\prime}-y_{2}\right)} \mathrm{d} S_{y}^{\prime} \mathrm{d} S_{y}
\end{array}
$$

where the following notations have been introduced:

$$
K_{1}=\frac{\omega\left(x_{1}-M_{x} S_{0}\right)}{\beta_{x}^{2} c_{0} S_{0}} \quad \text { and } \quad K_{2}=\frac{\omega x_{2}}{c_{0} S_{0}}
$$

To this point, the derivation of the model is identical to the one by Amiet [41]. However, since our intention is to use the model for the SP frequency spectrum devised in the previous section (in contrast to Amiet's theory using a wavenumber spectrum), a slightly different approach for the model derivation is required. Following Amiet's derivation, the radiating SP is decomposed into the sum of an incident pressure wave and a contribution due to the trailing edge scattering. In his case, the incident pressure is generated by the attached turbulent boundary layer flow over the airfoil. In our case, it is the SP generated by the stalled flow. It is thereby assumed that the scattered pressure field does not perturbate the measured SP spectra presented earlier in this work. This is largely a valid assumption since scattering effects are confined to the very 
vicinity of the trailing edge [37] and our measurements were always performed relatively far from it, not least for obvious practical reasons.

Two model versions are proposed below. The first one (referred to as 'MODA') closely follows the approach of Amiet which defines the incident pressure field based on its knowledge close to the trailing edge, assuming an exponential growth of the SP fluctuations toward the trailing edge. The second version (referred to as 'MODB') explicitly prescribes the incident pressure all along the airfoil chord using the SP model proposed in Section 6.3.

\subsection{Model MODA Based on Amiet's Incident Pressure Wave}

According to Amiet [41], the total SP is decomposed into an incident and a scattered field as:

$$
\tilde{p}(\omega, \boldsymbol{y})=\tilde{p}_{i}(\omega, \boldsymbol{y})+\tilde{p}_{s}(\omega, \boldsymbol{y})
$$

which are both related to their respective wavenumber spectra in the span direction as:

$$
\tilde{p}_{f}(\omega, \boldsymbol{y})=\int_{-\infty}^{+\infty} \hat{p}_{f}\left(\omega, y_{1}, k_{2}\right) \mathrm{e}^{+\mathrm{i} k_{2} y_{2}} \mathrm{~d} k_{2} \quad(f=i, s)
$$

Assuming frozen turbulence, the scattered pressure can be expressed as:

$$
\tilde{p}_{s}(\omega, \boldsymbol{y})=\frac{1}{U_{\mathrm{cv}}} \int_{-\infty}^{+\infty} f\left(y_{1}, K_{c}, k_{2}\right) \hat{p}_{i, t e}\left(K_{c}, k_{2}\right) \mathrm{e}^{+\mathrm{i} k_{2} y_{2}} \mathrm{~d} k_{2}
$$

where $K_{c}=\omega / U_{\mathrm{cv}}$ is the convective wavenumber and $f\left(y_{1}, k_{1}, k_{2}\right)$ is the response function derived from the solution of the Schwartzschild problem which is used to formulate the 775 scattering phenomenon at the trailing edge $[41,42]$. Its derivation was further extended by Roger and Moreau [43] to account for the back-scattering from the leading edge and this latter formulation is implemented in the present model. Here and in the following, the subscript ,te always refers to a quantity evaluated at the trailing edge, that is at $y_{1}=C$. Furthermore, following Amiet's assumption the incident pressure field is assumed to grow exponentially during its convection toward the trailing edge where it reaches its maximum. Therefore, together with the frozen turbulence assumption, it takes the following form:

$$
\tilde{p}_{i}(\omega, \boldsymbol{y})=\frac{1}{U_{\mathrm{cv}}} \int_{-\infty}^{+\infty} \mathrm{e}^{-\varepsilon K_{c}\left(C-y_{1}\right)} \hat{p}_{i, t e}\left(K_{c}, k_{2}\right) \mathrm{e}^{+\mathrm{i} k_{2} y_{2}} \mathrm{~d} k_{2}
$$


where $\varepsilon$ measures the rate of this exponential growth. In Amiet's derivation the quantity $\varepsilon$ does not explicitly appear in the model formulation but the final result for the lift response function is modified [44]. Casper and Farassat [45] used the following value $\varepsilon=1.5$. Moreau et al [46] proposed $\varepsilon=4 /\left(K_{c} C\right)$ and this latter relationship is used in the present study as it is found to yield the best results. Introducing Eqs. (17) and (18) into Eq. (15) and introducing the following function:

$$
g\left(y_{1}, K_{c}, K_{1}, k_{2}\right)=\left(f\left(y_{1}, K_{c}, k_{2}\right)+\mathrm{e}^{-\varepsilon K_{c}\left(C-y_{1}\right)}\right) \mathrm{e}^{+\mathrm{i} K_{1} y_{1}}
$$

yields:

$$
\begin{aligned}
& S_{a}^{(A)}(\omega, \boldsymbol{x})=\left(\frac{\omega x_{3}}{4 \pi c_{0} S_{0}^{2}}\right)^{2} \frac{1}{U_{\mathrm{cv}}} \iint_{S_{y}} \iint_{S_{y}^{\prime}} \iint_{-\infty}^{+\infty} \cdots \\
&<\hat{p}_{i, t e}\left(K_{c}, k_{2}\right) \cdot \hat{p}_{i, t e}^{*}\left(K_{c}, k_{2}^{\prime}\right)> g\left(y_{1}, K_{c}, K_{1}, k_{2}\right) \cdot g^{*}\left(y_{1}^{\prime}, K_{c}, K_{1}, k_{2}^{\prime}\right) \cdots \\
& \times \mathrm{e}^{\mathrm{i}\left(k_{2} y_{2}-k_{2}^{\prime} y_{2}^{\prime}\right)} \mathrm{e}^{-\mathrm{i} K_{2}\left(y_{2}^{\prime}-y_{2}\right)} \mathrm{d} S_{y}^{\prime} \mathrm{d} S_{y} \mathrm{~d} k_{2} \mathrm{~d} k_{2}^{\prime}
\end{aligned}
$$

where the superscript ${ }^{(A)}$ refers to the model MODA. Introducing the spanwise wavenumber power spectrum of the incident pressure field at the trailing edge yields:

$$
\begin{aligned}
S_{a}^{(A)}(\omega, \boldsymbol{x})= & \left(\frac{\omega x_{3}}{4 \pi c_{0} S_{0}^{2}}\right)^{2} \frac{1}{U_{\mathrm{cv}}} \iint_{S_{y}} \iint_{S_{y}^{\prime}} \int_{-\infty}^{+\infty} \cdots \\
& S_{p p, t e}\left(K_{c}, k_{2}\right) g\left(y_{1}, K_{c}, K_{1}, k_{2}\right) \cdot g^{*}\left(y_{1}^{\prime}, K_{c}, K_{1}, k_{2}\right) \cdots \\
& \times \mathrm{e}^{-\mathrm{i} k_{2}\left(y_{2}^{\prime}-y_{2}\right)} \mathrm{e}^{-\mathrm{i} K_{2}\left(y_{2}^{\prime}-y_{2}\right)} \mathrm{d} S_{y}^{\prime} \mathrm{d} S_{y} \mathrm{~d} k_{2}
\end{aligned}
$$

This power spectrum is transformed back to the physical space by inverse Fourier tranform in the span direction as:

$$
S_{p p, t e}\left(K_{c}, k_{2}\right)=\frac{1}{2 \pi} \int_{-\infty}^{+\infty} S_{p p, t e}\left(K_{c}, \eta\right) \mathrm{e}^{-\mathrm{i} k_{2} \eta} \mathrm{d} \eta
$$

where $\eta=y_{2}-y_{2}^{\prime}$. Assuming that the cross-spectrum of the SP can be separated into:

$$
S_{p p, t e}\left(K_{c}, \eta\right)=S_{p p, t e}\left(K_{c}\right) \gamma_{2, t e}\left(K_{c}, \eta\right)
$$


yields:

$$
\begin{aligned}
& S_{a}^{(A)}(\omega, \boldsymbol{x})=\left(\frac{\omega x_{3}}{4 \pi c_{0} S_{0}^{2}}\right)^{2} \frac{1}{U_{\mathrm{cv}}} \iint_{S_{y}} \iint_{S_{y}^{\prime}} \int_{-\infty}^{+\infty} \cdots \\
& S_{p p, t e}\left(K_{c}\right) \Gamma_{2, t e}\left(K_{c}, k_{2}\right) g\left(y_{1}, K_{c}, K_{1}, k_{2}\right) \cdot g^{*}\left(y_{1}^{\prime}, K_{c}, K_{1}, k_{2}\right) \cdots \\
& \quad \times \mathrm{e}^{-\mathrm{i} k_{2}\left(y_{2}^{\prime}-y_{2}\right)} \mathrm{e}^{-\mathrm{i} K_{2}\left(y_{2}^{\prime}-y_{2}\right)} \mathrm{d} S_{y}^{\prime} \mathrm{d} S_{y} \mathrm{~d} k_{2}
\end{aligned}
$$

where $\Gamma_{2}$ is the Fourier transform of the coherence function $\gamma_{2}$ as introduced in Section 5.3.

Changing the order in which integrals are performed in Eq. (20), the following three-fold integral appears as the most inner one:

$$
\begin{aligned}
I=\int_{-\infty}^{+\infty} \Gamma_{2, t e}\left(K_{c}, k_{2}\right) g\left(y_{1},\right. & \left.K_{c}, K_{1}, k_{2}\right) \cdot g^{*}\left(y_{1}^{\prime}, K_{c}, K_{1}, k_{2}\right) \cdots \\
& \times\left(\iint_{-L / 2}^{L / 2} \mathrm{e}^{-\mathrm{i}\left(K_{2}-k_{2}\right)\left(y_{2}^{\prime}-y_{2}\right)} \mathrm{d} y_{2}^{\prime} \mathrm{d} y_{2}\right) \mathrm{d} k_{2}
\end{aligned}
$$

where the Fourier transform of the box function can be recognized twice within the parentheses. Therefore, this integral can be reformulated as:

$$
\begin{aligned}
I=\int_{-\infty}^{+\infty} \Gamma_{2, t e}\left(K_{c}, k_{2}\right) g\left(y_{1}, K_{c}, K_{1}, k_{2}\right) & \cdot g^{*}\left(y_{1}^{\prime}, K_{c}, K_{1}, k_{2}\right) \cdots \\
& \times L^{2}\left(\frac{\sin \left(\frac{\left(K_{2}-k_{2}\right) L}{2}\right)}{\frac{\left(K_{2}-k_{2}\right) L}{2}}\right)^{2} \mathrm{~d} k_{2}
\end{aligned}
$$

Further assuming that the airfoil is acoustically compact in the span direction yields:

$$
I=2 \pi L \Gamma_{2, t e}\left(K_{c}, K_{2}\right) g\left(y_{1}, K_{c}, K_{1}, K_{2}\right) \cdot g^{*}\left(y_{1}^{\prime}, K_{c}, K_{1}, K_{2}\right)
$$

Introducing this result in Eq. (20), it now reads:

$$
\begin{aligned}
S_{a}^{(A)}(\omega, \boldsymbol{x})=\left(\frac{\omega x_{3}}{4 \pi c_{0} S_{0}^{2}}\right)^{2} \frac{2 \pi L}{U_{\mathrm{cv}}} \iint_{0}^{C} S_{p p, t e}\left(K_{c}\right) \Gamma_{2, t e}\left(K_{c}, K_{2}\right) \cdots \\
\quad \times g\left(y_{1}, K_{c}, K_{1}, K_{2}\right) \cdot g^{*}\left(y_{1}^{\prime}, K_{c}, K_{1}, K_{2}\right) \mathrm{d} y_{1} \mathrm{~d} y_{1}^{\prime}
\end{aligned}
$$

As a final step, the wavenumber spectra in $K_{c}$ are transformed back to frequency spectra using the frozen turbulence assumption:

$$
\begin{aligned}
S_{a}^{(A)}(\omega, \boldsymbol{x})=\left(\frac{\omega x_{3}}{4 \pi c_{0} S_{0}^{2}}\right)^{2} 2 \pi L \iint_{0}^{C} S_{p p, t e}(\omega) \Gamma_{2, t e}\left(\omega, K_{2}\right) \cdots \\
\quad \times g\left(y_{1}, K_{c}, K_{1}, K_{2}\right) \cdot g^{*}\left(y_{1}^{\prime}, K_{c}, K_{1}, K_{2}\right) \mathrm{d} y_{1} \mathrm{~d} y_{1}^{\prime}
\end{aligned}
$$


where the function $g$ has been kept as a function of $K_{c}$ as it is a deterministic quantity noise spectrum can be further simplified as:

$$
S_{a}^{(A)}(\omega, \boldsymbol{x})=\left(\frac{\omega x_{3}}{4 \pi c_{0} S_{0}^{2}}\right)^{2} 2 \pi L\left|G\left(K_{c}, K_{1}, K_{2}\right)\right|^{2} S_{p p, t e}(\omega) \Gamma_{2, t e}\left(\omega, K_{2}\right)
$$

where the function $G$ is the equivalent of the lift function introduced in Amiet's model. It reads:

$$
G\left(K_{c}, K_{1}, K_{2}\right)=\int_{0}^{C} g\left(y_{1}, K_{c}, K_{1}, K_{2}\right) \mathrm{d} y_{1}
$$

and includes both the incident and scattered field contributions. It is here evaluated numerically instead of analytically as in Amiet's derivation [44]. Converged results are obtained using 100 points for the integration along the chord and the discretization is quadratically refined near the separation point.

\subsection{Model MODB Using Modelled Surface Pressure as Incident Pressure}

In this second version of a stall noise model, the total SP is decomposed again according to Eq. (16) and the scattered field contribution is still given by Eq. (17). However, the SP model introduced in Section 6.3 can be used to define the incident pressure field across the entire airfoil chord. Relating the incident pressure $\tilde{p}_{i}(\omega, \boldsymbol{y})$ to its wavenumber spectrum in the span direction and assuming frozen turbulence, it reads:

$$
\tilde{p}_{i}(\omega, \boldsymbol{y})=\frac{1}{U_{\text {cv }}} \int_{-\infty}^{+\infty} \mathrm{e}^{+\mathrm{i} K_{c}\left(C-y_{1}\right)} \hat{p}_{i}\left(y_{1}, K_{c}, k_{2}\right) \mathrm{e}^{+\mathrm{i} k_{2} y_{2}} \mathrm{~d} k_{2}
$$

which can replace Eq. (18) before being introduced in Eq. (15) as in the MODA model derivation. The equivalent of Eq. (19) for the MODB model now reads:

$$
\begin{aligned}
& S_{a}^{(B)}(\omega, \boldsymbol{x})=\left(\frac{\omega x_{3}}{4 \pi c_{0} S_{0}^{2}}\right)^{2} \frac{1}{U_{\mathrm{cv}}} \iint_{S_{y}} \iint_{S_{y}^{\prime}} \iint_{-\infty}^{+\infty} \cdots \\
&<\left(\hat{p}_{i}\left(y_{1}, K_{c}, k_{2}\right) \mathrm{e}^{+\mathrm{i} K_{c}\left(C-y_{1}\right)}+f\left(y_{1}, K_{c}, k_{2}\right) \hat{p}_{i, t e}\left(K_{c}, k_{2}\right)\right) \cdots \\
& \cdot\left(\hat{p}_{i}^{*}\left(y_{1}^{\prime}, K_{c}, k_{2}^{\prime}\right) \mathrm{e}^{-\mathrm{i} K_{c}\left(C-y_{1}^{\prime}\right)}+f^{*}\left(y_{1}^{\prime}, K_{c}, k_{2}^{\prime}\right) \hat{p}_{i, t e}^{*}\left(K_{c}, k_{2}^{\prime}\right)\right)>\cdots \\
& \quad \times \mathrm{e}^{\mathrm{i}\left(k_{2} y_{2}-k_{2}^{\prime} y_{2}^{\prime}\right)} \mathrm{e}^{-\mathrm{i} K_{2}\left(y_{2}^{\prime}-y_{2}\right)} \mathrm{e}^{-\mathrm{i} K_{1}\left(y_{1}^{\prime}-y_{1}\right)} \mathrm{d} S_{y}^{\prime} \mathrm{d} S_{y} \mathrm{~d} k_{2} \mathrm{~d} k_{2}^{\prime}
\end{aligned}
$$


The successive steps of the model derivation are analogous to those of the MODA model in Section 7.2, the main differences being:

- the function $f$ and the term $\mathrm{e}^{-\mathrm{i} K_{1}\left(y_{1}^{\prime}-y_{1}\right)}$ are explicitly kept in the derivation instead of introducing function $g$,

- Eq. (23) replaces Eq. (18),

- the trailing edge SP spectral term $<\hat{p}_{i, t e} \cdot \hat{p}_{i, t e}^{*}>$ in Eq. (19) is replaced by expectation terms resulting from the expansion of the term within angle brackets in Eq. (24).

As a result of the above-mentioned expansion, additional cross-correlation terms across $y_{1}$ appear and the following separated functional form is assumed for these terms:

$$
\begin{aligned}
<\hat{p}_{i}\left(y_{1}, K_{c}, k_{2}\right) \cdot \hat{p}_{i}^{*}\left(y_{1}^{\prime}, K_{c}, k_{2}^{\prime}\right)>=\cdots & \\
& S_{p p}\left(y_{1}, y_{1}^{\prime}, K_{c}\right) \gamma_{1}\left(y_{1}, y_{1}^{\prime}, \xi, K_{c}\right) \Gamma_{2}\left(y_{1}, y_{1}^{\prime}, K_{c}, k_{2}\right) \delta\left(k_{2}-k_{2}^{\prime}\right)
\end{aligned}
$$

where $y_{1}$ and $y_{1}^{\prime}$ may be equal to $C$ for terms involving $\hat{p}_{i, t e}, \xi=y_{1}-y_{1}^{\prime}$, and $\gamma_{1}$ is the coherence function as introduced in Section 5.2. In the above formula, a new notation has been introduced in the form of the SP power spectrum and the coherence functions depending simultaneously on $y_{1}$ and $y_{1}^{\prime}$. For symmetry purpose during the numerical evaluation of the model formula (see below), these functions are in practice defined using the geometric mean. For the SP power spectrum, it reads:

$$
S_{p p}\left(\omega, y_{1}, y_{1}^{\prime}\right)=\sqrt{S_{p p}\left(\omega, y_{1}\right) \cdot S_{p p}\left(\omega, y_{1}^{\prime}\right)}
$$

with analogous relationships for $\gamma_{1}$ and $\Gamma_{2}$. Note that $\gamma_{1}\left(\omega, y_{1}, y_{1}, 0, K_{c}\right)=1$. 
All calculations done, the final equation for MODB model equivalent of Eq. (21) reads:

$$
\begin{gathered}
S_{a}^{(B)}(\omega, \boldsymbol{x})=\left(\frac{\omega x_{3}}{4 \pi c_{0} S_{0}^{2}}\right)^{2} 2 \pi L \iint_{0}^{C} \ldots \\
\quad\left(S_{p p}\left(\omega, y_{1}, y_{1}^{\prime}\right) \gamma_{1}\left(\omega, y_{1}, y_{1}^{\prime}, y_{1}-y_{1}^{\prime}\right) \Gamma_{2}\left(\omega, y_{1}, y_{1}^{\prime}, K_{2}\right) \mathrm{e}^{+\mathrm{i} K_{c}\left(y_{1}^{\prime}-y_{1}\right)} \ldots\right. \\
+S_{p p, t e}(\omega) \Gamma_{2, t e}\left(\omega, K_{2}\right) f\left(y_{1}, K_{c}, K_{2}\right) \cdot f^{*}\left(y_{1}^{\prime}, K_{c}, K_{2}\right) \cdots \\
+S_{p p}\left(\omega, y_{1}, C\right) \gamma_{1}\left(\omega, y_{1}, C, y_{1}-C\right) \Gamma_{2}\left(\omega, y_{1}, C, K_{2}\right) \cdots \\
\quad \times f^{*}\left(y_{1}^{\prime}, K_{c}, K_{2}\right) \mathrm{e}^{+\mathrm{i} K_{c}\left(C-y_{1}\right)} \ldots \\
+S_{p p}\left(\omega, C, y_{1}^{\prime}\right) \gamma_{1}\left(\omega, C, y_{1}^{\prime}, y_{1}^{\prime}-C\right) \Gamma_{2}\left(\omega, C, y_{1}^{\prime}, K_{2}\right) \cdots \\
\left.\quad \times f\left(y_{1}, K_{c}, K_{2}\right) \mathrm{e}^{-\mathrm{i} K_{c}\left(C-y_{1}^{\prime}\right)}\right) \cdots \\
\times \mathrm{e}^{-\mathrm{i} K_{1}\left(y_{1}^{\prime}-y_{1}\right)} \mathrm{d} y_{1} \mathrm{~d} y_{1}^{\prime}
\end{gathered}
$$

This equation is not further simplified as done for the MODA model in Eq. (22) and

the two-fold integration over $y_{1}$ and $y_{1}^{\prime}$ is performed numerically (see Section 7.2).

\section{Validation of Models}

In this section, the SP and stall noise models proposed earlier are compared with measurements performed in various anechoic wind tunnels.

\subsection{NACA-64-618 Measurements in Virginia Tech Wind Tunnel [27]}

The first validation study compares the noise models developed in the previous section with far-field noise measurements performed in the Virginia Tech anechoic wind tunnel [27]. The facility and the experimental conditions are introduced in Section 2. The far-field noise was measured with a microphone array located at $1.62 \mathrm{~m}$ above the center of the airfoil section and the noise was integrated over a span length $L=0.6 \mathrm{~m}$. The results are presented at $R e=1.0 \times 10^{6}, 1.5 \times 10^{6}$ and $1.9 \times 10^{6}$ and with ZZ tape for the second case, and at $\alpha=10^{\circ}, 12^{\circ}$ and $14^{\circ}$, though the measured noise spectra are also displayed for $\alpha=6^{\circ}$ and $8^{\circ}$ for reference. The results for models MODA and MODB are compared to the experimental data in Figs. 24(a) and (b), respectively. In 
the model calculations, the separation location is located for each individual case using

the measured pressure coefficient distribution as explained in Section 3.

The MODA model predicts the measured decrease of spectral slope toward higher frequencies, but it underestimates significantly the measurements for Reynolds numbers larger than $R e \geq 1.5 \times 10^{6}$. The spectral energy levels are equivalent to the levels observed when the flow is still attached (i.e. for $\alpha=6^{\circ}$ and $8^{\circ}$ ). Moreover, the model predicts an opposite trend compared to the increase of measured spectral energy as the AoA increases. As for the MODB model, it is nearly insensitive to the effect of the AoA through the specification of the separation point (see above), but the spectral energy levels are closer to the measured ones.

As a conclusion, the model is not performing very well in this case. However, it should be noted that the noise measurement technique is limited to frequencies above $500 \mathrm{~Hz}$ and therefore the spectral hump characteristic of stall at low frequencies is not captured.

\subsection{NACA0012 Measurements by Brooks et al [8]}

Brooks et al [8] realized a series of experiments in a low-turbulence jet located in an anechoic chamber using different configurations of NACA0012 airfoil section models. Acoustic measurements were performed in various flow conditions and this campaign resulted in the well-known and so-called BPM model for airfoil noise predictions. In our case, only the measurements performed with a $22.86 \mathrm{~cm}$ chord airfoil at inflow velocities $U_{\infty}=31.7,39.6,55.5$ and $71.3 \mathrm{~m} / \mathrm{s}$, and at a geometric AoA $\alpha=19.8^{\circ}$ are considered. Assuming standard ambient temperature and atmospheric pressure, the approximated Reynolds numbers are respectively equal to $R e=0.5 \times 10^{6}, 0.6 \times 10^{6}, 0.85 \times 10^{6}$ and $1.1 \times 10^{6}$. Since the present stall noise model depends on the separation location and this quantity was not documented in the campaign report [8], it has to be inferred. Our approach consists in tuning the separation location so that the peak frequency at which the far-field noise spectrum reaches its maximum value approximately coincides with the measured one for each individual test case. The tuned values are $x / C=0.6,0.5,0.4$ and 0.3 for the respective test cases. However, the energy content (i.e. absolute level) 
and the shape of the modelled spectra are entirely determined by the model formulation proposed earlier.

The modelled and measured far-field noise 1/3-octave band spectra are compared in Fig. 25. As it can be seen the peak spectrum levels are overpredicted at the lowest inflow velocities. Nevertheless, the change of spectral shape between lower frequencies $(f \lesssim 1000 \mathrm{~Hz})$ where a high energy hump is clearly observed and higher frequencies where the roll-off of the spectra becomes linear (in log-log scale) are well reproduced by the models MODA and MODB. At higher velocities, both models reproduce quite well the spectral peaks. The MODB model performs slightly better in this respect. Furthermore, it can be noticed that the energy levels at higher frequencies (i.e. $f \gtrsim 1000 \mathrm{~Hz}$ ) are also quite well predicted in all cases.

An interesting fact that should be pointed out is the interpretation of the spectra at high frequencies, i.e. where the linear roll-off observed above is established, in the BPM [8] analysis. Indeed, according to their model this part of the spectra is attributed to the contribution of trailing edge noise from the pressure side of the airfoil. Indeed, it is well known that the main variables characterizing trailing edge noise are the boundary layer thickness and the velocity profile. Thus, a thinner boundary layer on the pressure side suggests that its contribution as trailing edge noise is dominating in the high frequency range. However, in the present model the high frequency linear roll-off part of the spectra directly results from the SP model that was proposed in Section 6.3 combined with the stall noise models derived in Sections 7.2 or 7.3. Therefore, the model results do not involve any contribution from the pressure side turbulent boundary layer, unless the measured SP on the suction side would be driven by turbulent vortices from the pressure side boundary layer, which seems rather unrealistic.

\subsection{NACA-0012 Airfoil Measurements by Moreau et al [11]}

The NACA-0012 airfoil was also measured by Moreau et al [11] in the wind tunnel at ECL (Lyon, France) which consists of a $50 \mathrm{~cm}$ wide rectangular open jet blowing into an anechoic chamber. In the considered cases, the airfoil model has $8 \mathrm{~cm}$ chord and is held between two plates $13 \mathrm{~cm}$ apart for the far-field noise measurement, and a 
$10 \mathrm{~cm}$ chord with a span of $30 \mathrm{~cm}$ for the SP spectra. The test run considered here is performed at $\alpha=15^{\circ}$ and $18^{\circ}$, respectively, and a Reynolds number $R e=0.16 \times 10^{6}$ which is relatively low in comparison to the set of experimental data considered in the present study. The separation location is not specified in the reference and is assumed equal to $x_{\mathrm{sep}} / C=10 \%$ in the SP and noise models.

The measured SP spectrum at $x / C=96 \%$ and far-field noise spectrum at $1.75 \mathrm{~m}$ above the mid-span of the airfoil are displayed in Fig. 26 together with the results of the present models as proposed in Sections 6.3 and 7.2-7.3. The measurements are overestimate by the SP model above a Strouhal approximately equal to 1 and slightly underetimated below. Concerning the far-field noise, the MODB model predicts quite well the measurements above $500 \mathrm{~Hz}$, but both models completely miss the continous increase of noise sound pressure levels toward low frequencies below. It should be noted here that the present models are essentially identical to Amiet's formulation [41] which is formally strictly valid at high-frequencies only. This may explain the above discrepancies. Note also that the measured airfoil chord is quite smaller than those considered in the present study. Therefore, the airfoil is probably becoming acoustically compact at these low frequencies.

\subsection{Airfoil Measurements by Christophe et al [12]}

The next model validation uses measurement data from a study by Christophe et al [12] Their measurements were performed in the wind tunnel at ECL (Lyon, France) which consists of a $50 \mathrm{~cm}$ wide rectangular open jet blowing into an anechoic chamber. The CD airfoil model has $13.4 \mathrm{~cm}$ chord and is held between two plates $25 \mathrm{~cm}$ apart. The test run considered here is performed at $\alpha=15^{\circ}$ and a Reynolds number $R e=0.16 \times 10^{6}$ which is relatively low in comparison to the set of experimental data considered in the present study. Since the CD airfoil has a sharp leading edge and the AoA being relatively large, it is assumed that separation occurs near the leading edge and $x_{\text {sep }} / C=1 \%$ is enforced in the SP and noise models.

The measured SP spectrum at $x / C=98 \%$ and far-field noise spectrum at $2 \mathrm{~m}$ above the mid-span of the airfoil are displayed in Fig. 27 together with the results of the present 
models as proposed in Sections 6.3 and 7.2-7.3. The SP model reproduces very well the measurements up to $f=3 \mathrm{kHz}$. At higher frequencies, the measured spectrum decreases significantly while the model predicts the same linear roll-off as at lower frequencies. Concerning the far-field noise, the MODB model predicts correct spectral levels up to $f=300 \mathrm{~Hz}$ while the MODA model underpredicts the measurements, and vice-versa above this frequency. This peculiar behavior of the models remains unexplained, but remind that the present model has been designed from measurement data acquired at higher Reynolds numbers than the one considered here. The remarks formulated in the previous section concerning the validity of the present model at low frequencies and the fact that the measured airfoil becomes acoustically compact do also apply here.

\subsection{Measurements at AWB Wind Tunnel by DLR [13, 14]}

The Acoustic Wind tunnel Braunschweig is an anechoic facility with an open-jet configuration. The airfoil model is a $300 \mathrm{~mm}$ chord and $800 \mathrm{~mm}$ span DU-96-W-180 airfoil and it was measured at an inflow speed of $60 \mathrm{~m} / \mathrm{s}$, resulting in a Reynolds number of $1.2 \times 10^{6}$ at various AoAs. The measurements considered here involve two sets of data. In the first case, the airfoil was equipped with SP microphones [14], and in the second case far-field noise was measured [13].

These measurements are compared with the present models in Fig. 28. The SP are compared at two chord locations $x / C=88 \%$ and $96 \%$, and at two AoAs $\alpha=8.7^{\circ}$ and $10.3^{\circ}$. Once again, the separation location can be tuned so that the spectrum peak frequency coincides with the measurements. At the highest AoA, a good agreement is found for $x_{\mathrm{sep}} / C=60 \%$ and $70 \%$ for the two previous chord locations, respectively, suggesting that separation may be located between these two separation points. For the lowest AoA, the model largely over-estimates the mesurements. However, the different linear spectral roll-off above $\omega \gtrsim 10^{4} \mathrm{rad} / \mathrm{s}$ for the spectra at this AoA suggests that stall is not fully established. It may therefore indicate that the present model is not valid in early stall conditions. Note that Suryadi and Herr [14] also interpret this part of the spectrum as a contribution from the suction side (see discussion at the end of Section 8.2). 
The far-field noise is analyzed next. As observed in Fig. 28, both models MODA and MODB over-estimate the measurements by a few dBs at higher frequencies (Note that the measurement data displayed in Schuele and Rossignol [13] are two-sided and have been transformed to one-sided spectra here). Furthermore, model MODA predicts an early drop-off toward higher-frequencies $(f \gtrsim 6 \mathrm{kHz})$ while MODB predicts the correct slope for most of the high frequency part of the spectrum. The model results are plotted for various separation locations. As separation moves further upstream, the model results indicate that the spectral hump is moving to lower frequency as expected and in accordance with the modeling results by Schuele and Rossignol [13]. Their model does not account for the linear roll-off at higher frequencies (see related respectively. The results are plotted with errorbars in Fig. 29. It is observed that the model MODB is sensitive to the convection velocity in the high frequency range, while 
model MODA remains nearly insensitive. The model MODA is sensitive to the corre995 lation length in a short intermediate frequency range, and both models are sensitive to the separation location toward low frequencies. 

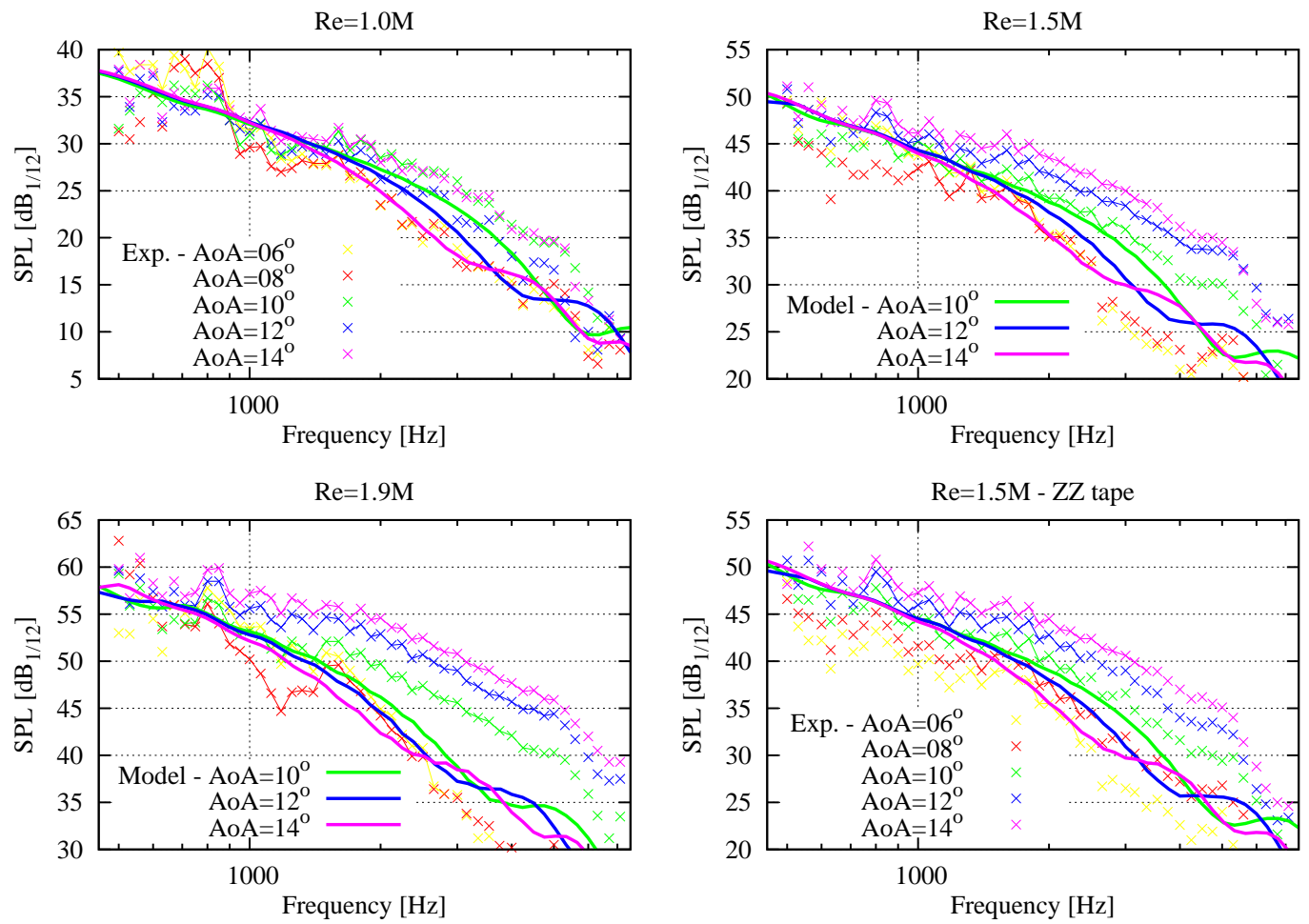

(a) MODA model
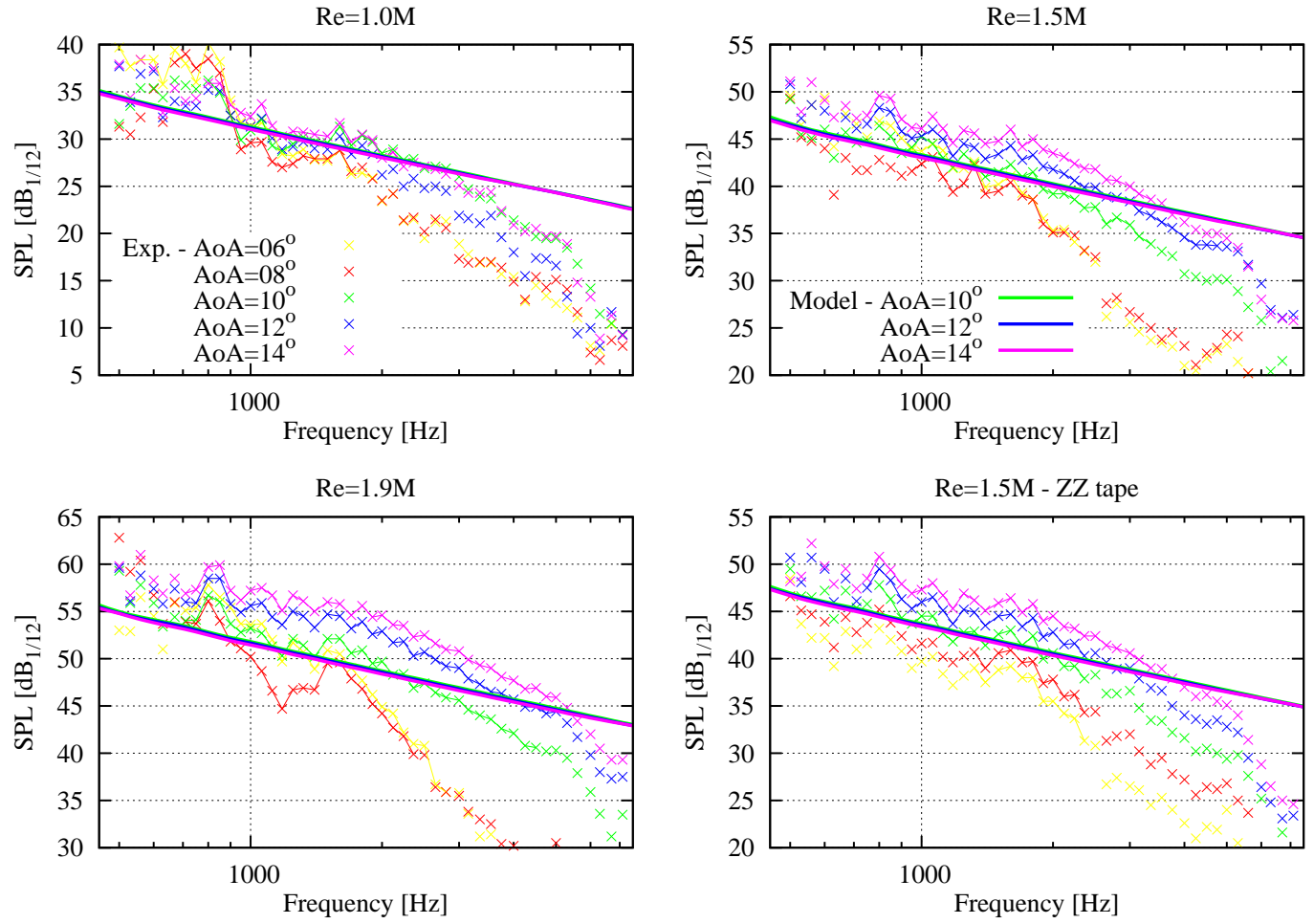

(b) MODB model

Figure 24: Comparison with Virginia Tech Noise Measurements [27] (Lines with points: trustworthy noise measurements; Points only: less reliable noise measurements) 

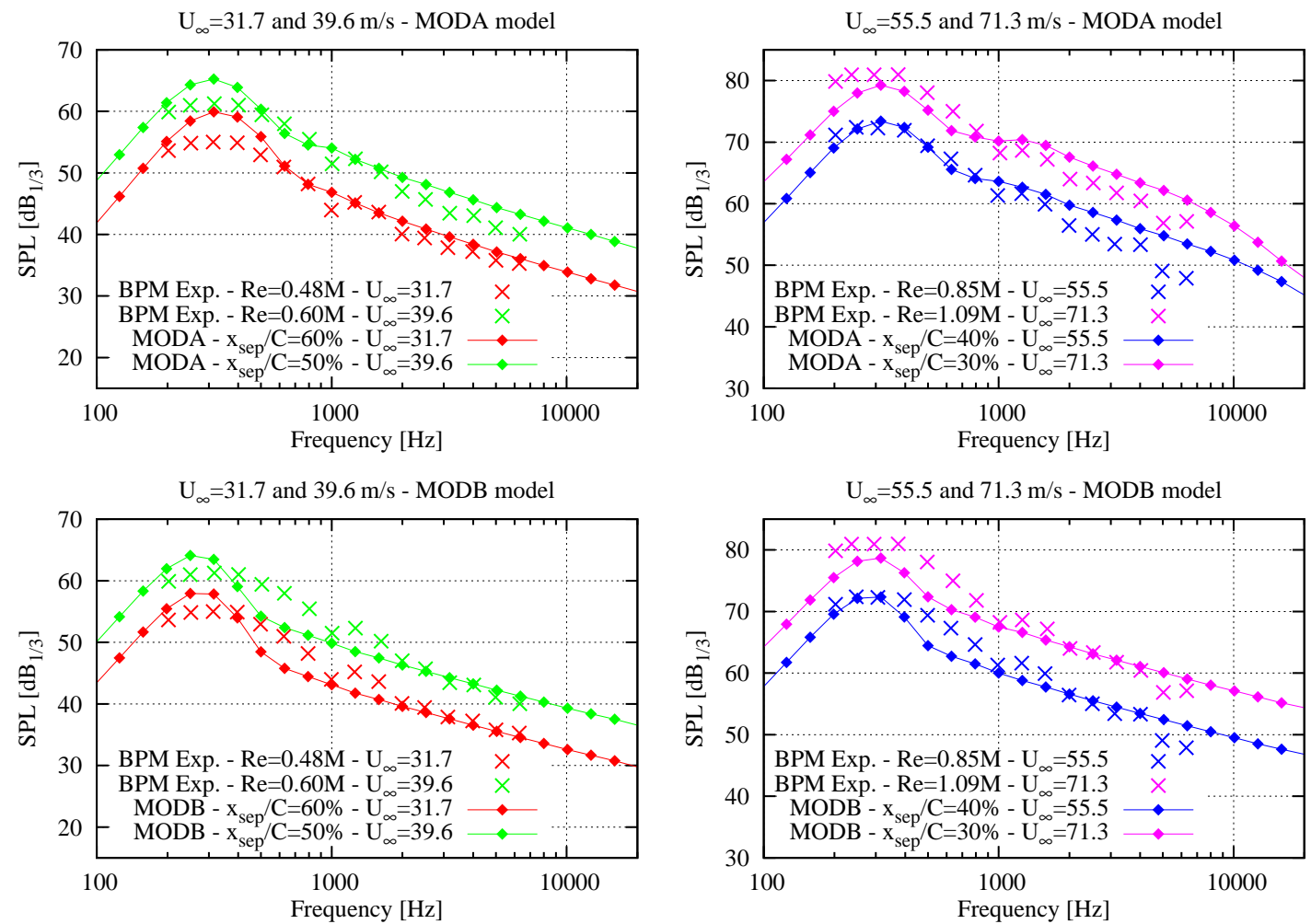

Figure 25: Comparison with NACA-0012 stall noise measurements [8] (Top: MODA; Bottom: MODB; Left: Low inflow velocities; Right: High inflow velocities)

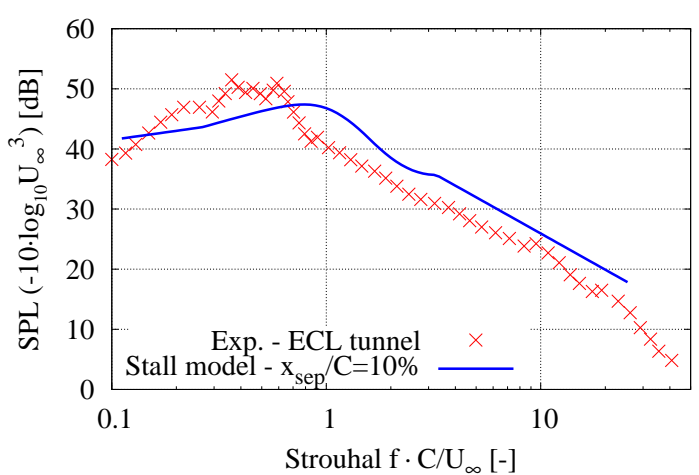

(a) SP spectra

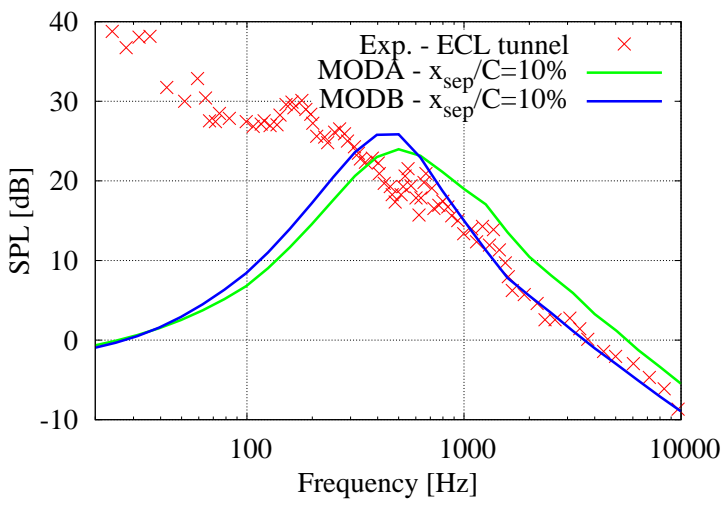

(b) Far-field noise

Figure 26: NACA-0012 airfoil - Comparison with ECL measurements [11] 

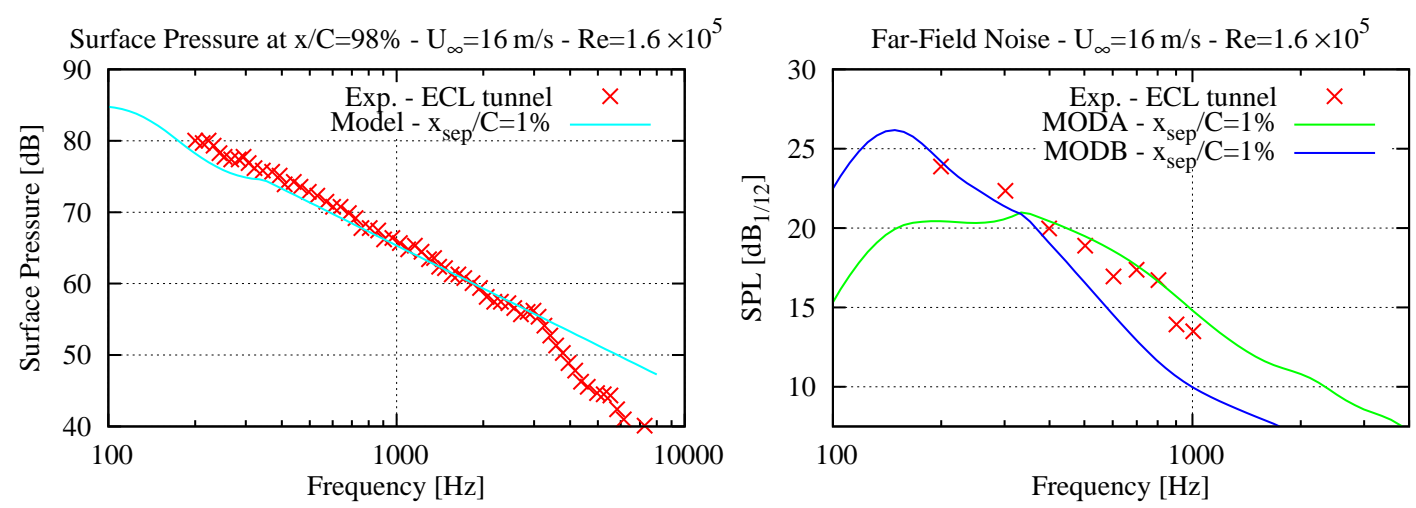

Figure 27: CD airfoil - Comparison with ECL measurements [12] (Left: SP; Right: far-field noise)
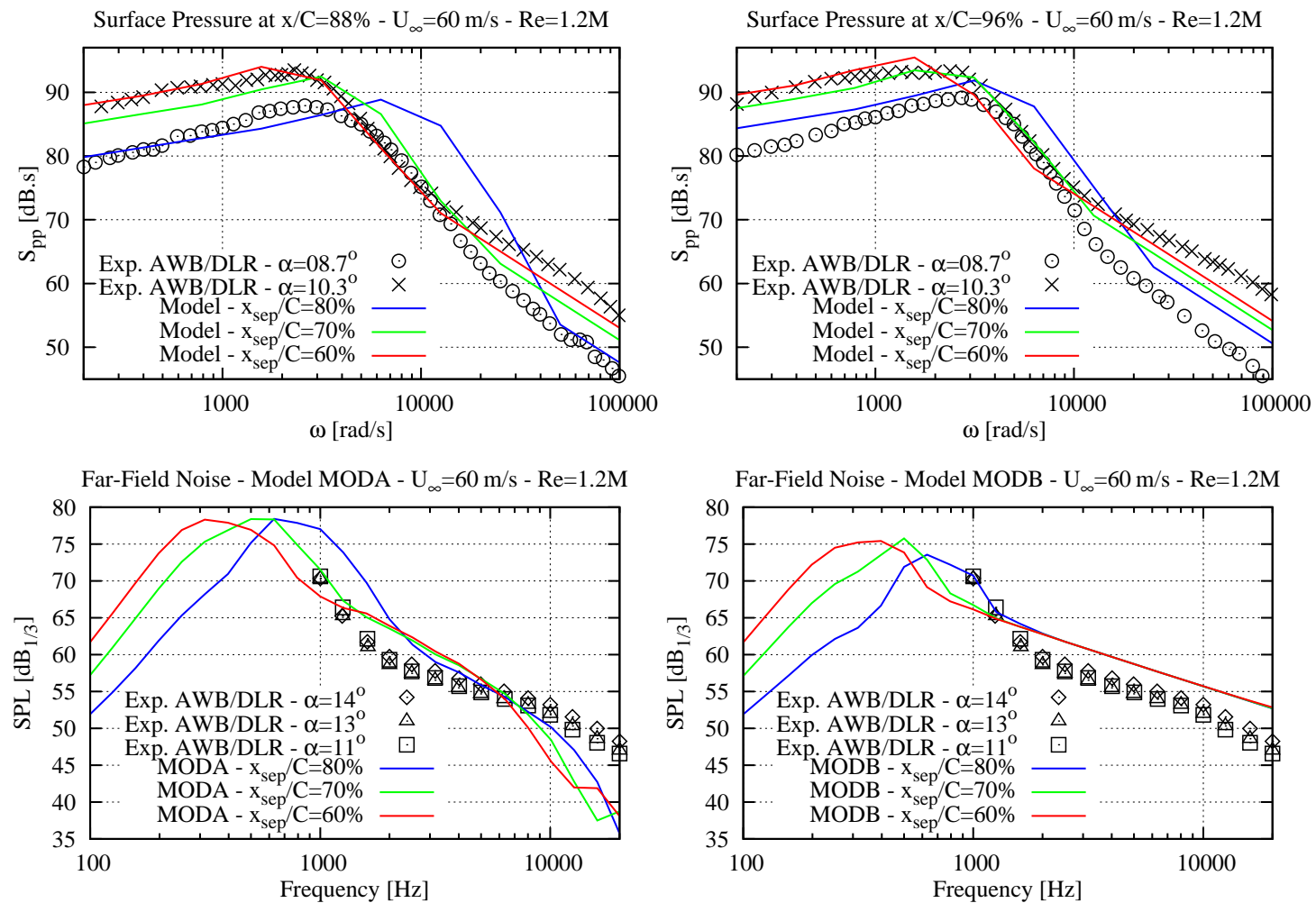

Figure 28: Comparison with DLR/AWB measurements (Top: SP [14]; Bottom: far-field noise [13]) 

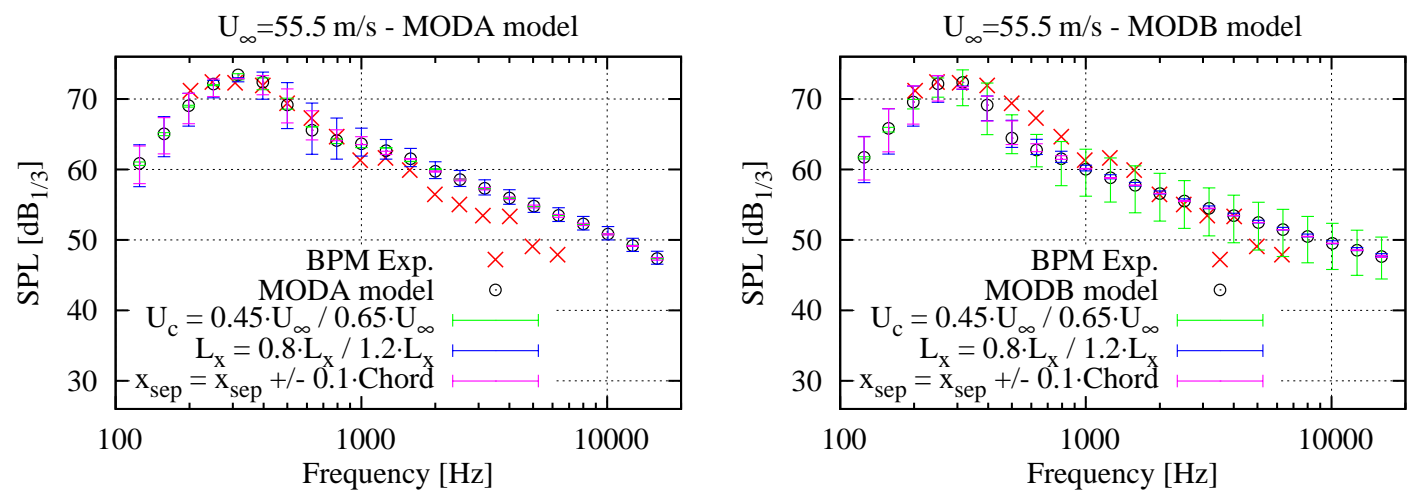

Figure 29: Sensivity of MODA (left) and MODB (right) models to input parameters NACA0012 airfoil at $U_{\infty}=55.5 \mathrm{~m} / \mathrm{s}$ as in [8] 


\section{Discussion and Conclusions}

In this article, measurements from microphones flush-mounted on various airfoils are used to develop an empirical model for the SP turbulent fluctuations within the detached flow region in stalled conditions, and subsequently a stall noise model.

It is shown in Section 4 that the convection velocity decreases as approaching the separation point from upstream and stabilizes to a nearly constant value once the flow is fully separated. Therefore, the separation location can be visually inferred from these plots. It was found that the inferred locations approximately coincide with those deduced from the static pressure coefficient distributions in Section 3. It also appears that the chordwise correlation length increases linearly with increasing distance from the separation location downstream in the separated flow region.

Analysis of the measured SP shows that microphones located in the separated region experience a large increase of the SP spectral energy in the form of a broad and high-energy hump in comparison to the attached flow. The peak frequency and the spectral energy level of this hump vary significantly as a function of the AoA and of the microphone location.

It is found that the above flow quantities can be scaled. A low and a high Reynolds number regimes for the scaling of the chordwise correlation lengths and the SP spectra could be identified. The chordwise correlations lengths as a function of distance from separation location exhibit different slopes in the two regimes and the SP spectra scales with different velocity exponents. The Reynolds number at which the transition between these two regimes occurs is approximated between $2 \times 10^{6}$ and $3 \times 10^{6}$.

This analysis also shows that the SP spectra scale as $U_{\infty}^{3}$ for low Reynolds numbers which is in agreement with earlier measurements [11]. At high Reynolds numbers, the present study shows that the spectra scale as $U_{\infty}^{1 / 2}$. These scalings apply at low frequencies where a spectral hump characteristic of stall is observed. Separately, at higher frequencies a scaling independent of Reynolds number but based on the distance of the measuring microphone to the separation point appears to be more appropriate. The transition between these two frequency ranges also obey its own scaling rule. 
The above scalings are used to define an empirical model for the SP based on a series of curve fitting. The resulting model is valid for Reynolds numbers ranging from $1 \times 10^{6}$ to $6 \times 10^{6}$ in accordance with the experimental data used to derive the above scalings. This SP model is combined with the trailing edge noise theory by Amiet [44] to develop a stall noise model. An alternative version of the original model derivation is also proposed. The models compare quite well with various anechoic wind tunnel noise measurements. Only in some cases does the latter model perform slightly better than the original one, even though it requires less assumptions about the SP definition. This aspect should be further investigated. It was also observed that the present model did fail to reproduce low frequency noise measurements for airfoils with small chord (see Sections 8.3-8.4) which may become acoustically compact at such frequencies. A possible extension of the present model would then be to implement a compact formulation as proposed by Moreau et al [11] for the low frequency part of the model.

One advantage of the present model is that the only input parameter is the separation point location, in addition to obvious geometric and flow parameters (i.e. airfoil chord and span, inflow velocity, air density and viscosity). Hence, if the separation location can be located using either measurements, simulation data or an empirical model, the proposed model is quite straightforward to implement. Nevertheless, it is not trivial to predict when stall separation initiates and it is at least as difficult to predict the exact separation location when stall occurs. Therefore, one of the main challenges to make this model more accurate and self-contained is the ability to predict both attributes.

To conclude, the proposed model is based on wind tunnel measurements and their scalings. The proposed scalings are not definitive and can surely be improved. So far, no physical rationale can be provided to justify them as, to the best author's knowledge, theoretical work that could describe detached airfoil flows from a statistical or spectral analysis point of view does not exist. The next step would then be to derive such a theory for the physics of turbulence within detached airfoil flows. 


\section{Acknowledgments}

Stéphane Rossignol for helpful discussions about our respective results.

The experimental campaigns presented in this paper were conducted under the 'DAN-AERO MW' projects (contract nos. ENS-33033-0074 and ENS-64009-0258) as well as the 'Low-Noise Airfoil' project (ENS-64009-0272), all funded by the Danish

Energy Agency (Energystyrelsen) as part of the EUDP demonstration program.

The authors would also like to thank LM Wind Power A/S for providing access to their wind tunnel facility in Lunderskov.

\section{References}

[1] J. B. Melvill, Stalling, Journal of the Royal Aeronautical Society 38 (1934) 7531065

[2] G. B. McCullough, D. E. Gaults, Examples of Three Representative Types of Airfoil-Section Stall at Low Speed, TN 2502, NACA, Ames Aeronautical Laboratory, Moffett Field (CA), 1951.

[3] D. N. Reilly, A Useful Method of Airfoil Stall Prediction, Journal of Aircraft 4 (1967) 567-568.

[4] B. S. Baldwin, H. Lomax, Thin Layer Approximation and Algebraic Turbulence Model for Separated Turbulent Flows, AIAA Paper 78-257.

[5] W. J. McCroskey, Unsteady Airfoils, Annual Review of Fluid Mechanics 14 (1982) $285-311$.

1075

[6] N. Alferez, I. Mary, E. Lamballais, Study of Stall Development Around an Airfoil by Means of High Fidelity Large Eddy Simulation, Flow, Turbulence and Combustion 91 (2013) 623-364. 
[7] J. G. Kocheemoolayil, W. Wolf, S. K. Lele, Large Eddy Simulation of Stall Noise, in: Proc. of the 20 ${ }^{\text {th }}$ AIAA/CEAS Aeroacoustics Conf., AIAA Paper 2014-3182, Atlanta, GA.

[8] T. F. Brooks, S. D. Pope, M. A. Marcolini, Airfoil Self-Noise and Prediction, NASA Reference Publication 1218, Langley Research Center, 1989.

[9] W. J. Devenport, R. A. Burdisso, A. Borgoltz, P. Ravetta, M. F. Barone, Aerodynamic and Acoustic Corrections for a Kevlar-Walled Anechoic Wind Tunnel, in: $16^{\text {th }}$ AIAA/CEAS Aeroacoustics Conf. (Proc.), AIAA Paper 2010-3749, Stockholm, Sweden.

[10] S. Moreau, M. Roger, Effect of Airfoil Aerodynamic Loading on Trailing-Edge Noise Sources, AIAA Journal 43 (2005) 41-52.

[11] S. Moreau, M. Roger, J. Christophe, Flow Features and Self-Noise of Airfoils Near Stall or Stall, in: Proc. of the $15^{\text {th }}$ AIAA/CEAS Aeroacoustics Conf., AIAA Paper 2009-3198, Miami, FL.

[12] J. Christophe, J. Anthoine, S. Moreau, Trailing Edge Noise of a ControlledDiffusion Airfoil at Moderate and High Angle of Attack, in: Proc. of the $15^{\text {th }}$ AIAA/CEAS Aeroacoustics Conf., AIAA Paper 2009-3196, Miami, FL.

[13] C. Y. Schuele, K.-S. Rossignol, Trailing-Edge Noise Modeling and Validation for Separated Flow Conditions, in: 19 ${ }^{\text {st }}$ AIAA/CEAS Aero-Acoustics Conference, AIAA Paper 2013-2008, Berlin, Germany.

[14] A. Suryadi, M. Herr, Wall Pressure Spectra on a DU96-W-180 Profile From Low to Pre-stall Angles of Attack, in: 21 ${ }^{\text {st }}$ AIAA/CEAS Aero-Acoustics Conference, AIAA Paper 2015-2688, Dallas, TX.

[15] D. Chase, Noise Radiated from an Edge in Turbulent Flow, AIAA J. 13 (1975) $1041-1047$. 
[16] W. K. Blake, Mechanics of Flow-Induced Sound and Vibration, Vol.I and II, volume in Applied Mathematics and Mechanics, Frenkiel, F.N. and Temple, G. (eds.), Academic Press, 1986.

[17] S. Nagarajan, S. Hahn, S. K. Lele, Prediction of Sound Generation by a Pitching Airfoil: A Comparison of RANS and LES, in: Proc. of the $12^{\text {th }}$ AIAA/CEAS Aeroacoustics Conf., AIAA Paper 2006-2516, Cambridge, MA.

[18] S. Oerlemans, M. G. Smith, P. White, S. von Hünerbein, A. King, B. Piper, M. Cand, A. Bullmore, B. Wilson, H. A. Madsen, A. Fischer, K. A. Kragh, Wind Turbine Amplitude Modulation: Research to Improve Understanding as to its Cause and Effects, Tech. Rep., RenewableUK, United Kingdom, 2013. (Available online: http://www.renewableuk.com/en/publications/reports.cfm/wind-turbineamplitude-modulation).

[19] M. Smith, A. J. Bullmore, M. M. Cand, R. Davis, Mechanisms of Amplitude Modulation in Wind Turbine Noise, in: Acoustics 2012, Conf. Proceedings, Nantes $(\mathrm{F})$.

[20] A. Laratro, M. Arjomandi, R. Kelso, B. Cazzolato, A Discussion of Wind Turbine Interaction and Stall Contributions to Wind Farm Noise, J. Wind Eng. Ind. Aerodyn. 127 (2014) 1-10.

[21] H. A. Madsen, F. Bertagnolio, A. Andreas, C. Bak, Correlation of Amplitude Modulation to Inflow Characteristics, in: Internoise 2014, Conference Proceedings, Melbourne, Australia.

[22] H. A. Madsen, C. Bak, U. S. Paulsen, M. Gaunaa, P. Fuglsang, J. Romblad, N. A. Olesen, P. Enevoldsen, J. Laursen, L. Jensen, The DAN-AERO MW Experiments - Final report, Tech. Rep. Risø-R-1726(EN), Risø-DTU, Roskilde, Denmark, 2010.

[23] H. D. Papenfuß, Aerodynamic Commissioning of the New Wind Tunnel at LM Glasfiber A/S (Lunderskov); Private Communication, 2006. Property of LM Glasfiber. 
[24] A. Fischer, Hot Wire Anemometer Turbulence Measurements in the Wind Tunnel of LM Wind Power, Tech. Rep. DTU-Wind-Energy-E0006(EN), DTU Wind Energy, Roskilde, Denmark, 2012. Available online: http://orbit.dtu.dk/en/publications/hot-wire-anemometer-turbulencemeasurements-in-the-wind-tunnel-of-lm-wind-power(5e3bea15-d2de-4c53-8d3c$59342 \mathrm{bb} 165 \mathrm{~b} 8) \cdot \mathrm{html}$.

[25] F. Bertagnolio, NACA0015 Measurements in LM Wind Tunnel and Turbulence Generated Noise, Tech. Rep. Ris $\varnothing-\mathrm{R}-1657(\mathrm{EN})$, Ris $\varnothing-D T U$, Roskilde, Denmark, 2008.

[26] W. Devenport, R. A. Burdisso, H. Camargo, E. Crede, M. Remillieux, M. Rasnick, P. V. Seeters, Aeroacoustic Testing of Wind Turbine Airfoils, Subcontract Report No. NREL/SR-500-43471, NREL, 2010. (Available online: http://www.nrel.gov/wind/pdfs/43471.pdf).

[27] A. Fischer, H. A. Madsen, F. Bertagnolio, Experimental Investigation of the Surface Pressure Field for Prediction of Trailing Edge Noise of Wind Turbine Aerofoils, International Journal of Aeroacoustics 14 (2015) 767-810.

[28] A. Fischer, F. Bertagnolio, C. Bak, H. A. Madsen, Surface Pressure Measurements on a NACA0015 Airfoil Compared to Computations with the TNO Trailing Edge Noise Model, in: Torque 2010 'The Science of Making Torque from Wind' Conference (EWEA), Conf. Proceedings, Crete, GR, pp. 81-92.

[29] R. Guastavino, On-site Microphone Calibration for 'Low Noise Airfoil' EUDP Project J.nr. 64009-0272, Tech. Rep. (Private Communication), Brüel \& Kjær Sound \& Vibration Measurement A/S, Nærum, Denmark, 2010.

[30] A. Fischer, Experimental Characterization of Airfoil Boundary Layers for Improvement of Aeroacoustic and Aerodynamic Modeling, PhD Thesis, DTU, Wind Energy Department, Roskilde, Denmark, 2011. Available online: http://orbit.dtu.dk/files/7688949/phd_thesis_main.pdf. 
[31] C. Bak, H. A. Madsen, P. Hansen, M. Rasmussen, P. Fuglsang, J. Romblad, N. A. Olesen, DAN-AERO MW: Measurement campaigns on the NM80 2.3MW wind turbine at Tjæreborg 2009, Tech. Rep. Risø-I-3046(EN), Risø-DTU, Roskilde, Denmark, 2010.

[32] C. Bak, H. A. Madsen, , M. Gaunaa, W. Skrzypinski, U. Paulsen, R. Møller, P. Hansen, M. Rasmussen, P. Fuglsang, DAN-AERO MW: Instrumentation of the NM80 2.3MW wind turbine including the LM $38.8 \mathrm{~m}$ blade and the meteorology mast at Tjæreborg, Tech. Rep. Ris $\varnothing-\mathrm{I}-3045(\mathrm{EN})$, Ris $\varnothing-D T U$, Roskilde, Denmark, 2010.

[33] H. A. Madsen, C. Bak, U. S. Paulsen, M. Gaunaa, P. Fuglsang, J. Romblad, N. A. Olesen, P. Enevoldsen, J. Laursen, L. Jensen, The DAN-AERO MW Experiments, in: $48^{\text {th }}$ AIAA Aerospace Sciences Meeting Including The New Horizons Forum and Aerospace Exposition (Proceedings), AIAA Paper 2010-645, Orlando (FL).

[34] C. Bak, H. A. Madsen, U. S. Paulsen, M. Gaunaa, P. Fuglsang, J. Romblad, N. A. Olesen, P. Enevoldsen, J. Laursen, L. Jensen, DAN-AERO MW: Details Aerodynamic Measurements on a Full-Scale MW Wind Turbine, in: 2010 European Wind Energy Conference - EWEC2010, Conf. Proceedings, Warsaw (Poland).

[35] F. Bertagnolio, H. A. Madsen, C. Bak, N. Troldborg, A. Fischer, Aerodynamic Noise Characterization of a Full-Scale Wind Turbine through High-Frequency Surface Pressure Measurements, International Journal of Aeroacoustics 14 (2015) 729-766.

[36] G. M. Corcos, The Structure of the Turbulent Pressure Field in Boundary-Layer Flows, J. Fluid Mech. 18 (1964) 353-378.

[37] T. F. Brooks, T. H. Hodgson, Trailing Edge Noise Prediction from Measured Surface Pressures, J. Sound Vib. 78 (1981) 69-117.

[38] S. Moreau, J. Christophe, M. Roger, LES of the Trailing-Edge Flow and Noise of 
a NACA0012 Airfoil Near Stall, in: Proc. of the Summer Program 2008, Center for Turbulence Research, Stanford Univ./NASA Ames, pp. 317-329.

1185

[42] R. K. Amiet, High-Frequency Thin Airfoil Theory for Subsonic Flow, AIAA J. 14 (1976) 1076-1082.

[43] M. Roger, S. Moreau, Back-Scattering Correction and Further Extensions of Amiet's Trailing-Edge Noise Model. Part 1: Theory, J. Sound Vib. 286 (2005) $477-506$.

[44] R. K. Amiet, Effect of the Incident Surface Pressure Field on Noise Due to Turbulent Flow Past a Trailing Edge, J. Sound Vib. 57 (1978) 305-306.

[45] J. Casper, F. Farassat, Broadband Trailing Edge Noise Predictions in the Time Domain, J. Sound Vib. 271 (2004) 159-176.

[46] S. Moreau, C. Schram, M. Roger, Diffraction Effects on Trailing-Edge Noise Measured in an Open-Jet Anechoic Wind Tunnel, in: Proc. of the $13^{\text {th }}$ AIAA/CEAS Aeroacoustics Conf., AIAA Paper 2007-3706, Roma, Italy. 